



\title{
Guide pour la coélaboration de scénarios Vers la compréhension partagée et l'action commune pour la réforme et la sécurité du foncier forestier
}

\author{
Robin Bourgeois \\ Centre de coopération internationale en recherche agronomique pour le développement (CIRAD) \\ The Centre for the Study of Governance Innovation (Govlnn) \\ Nining Liswanti \\ CIFOR \\ Concepta Mukasa \\ Association of Uganda Professional Women in Agriculture and Environment (AUPWAE)
}

Alejandra Zamora

Université nationale d'agronomie - La Molina (UNALM)

Tuti Herawati

CIFOR

Forestry and Environment Research, Development and Innovation Agency (FOERDIA)

lliana Monterroso

CIFOR

Baruani Mshale

CIFOR

Mani Ram Banjade

CIFOR

Esther Mwangi

CIFOR

Anne Larson

CIFOR

Centre de recherche forestière internationale (CIFOR) 
(c) 2021 Centre de recherche forestière internationale (CIFOR)

Le contenu de cette publication est soumis à une licence des Creative Commons Attribution 4.0 International (CC BY 4.0), http://creativecommons. org/licenses/by/4.0/

ISBN 978-602-387-154-4

DOI: $10.17528 /$ cifor/007984

Bourgeois R, Liswanti N, Mukasa C, Zamora A, Herawati T, Monterroso I, Mshale B, Banjade MR, Mwangi E et Larson A. 2021. Guide pour la coélaboration de scénarios : Vers la compréhension partagée et l'action commune pour la réforme et la sécurité du foncier forestier. Bogor, Indonésie : CIFOR.

Traduction de : Bourgeois R, Liswanti N, Mukasa C, Zamora A, Herawati T, Monterroso I, Mshale B, Banjade MR, Mwangi E and Larson A. 2017. Guide for co-elaboration of scenarios: Building shared understanding and joint action for reform and security of forest tenure. Bogor, Indonesia: CIFOR.

CIFOR

Jl. CIFOR, Situ Gede

Bogor Barat 16115

Indonesia

$\mathrm{T}+62(251) 8622-622$

$\mathrm{F}+62(251) 8622-100$

E cifor@cgiar.org

\section{cifor.org/fr}

Nous remercions tous nos partenaires financiers qui ont soutenu ce travail de recherche par leur contribution au fonds du CGIAR. La liste complète des donateurs du Fonds du CGIAR est consultable sur http:// www.cgiar.org/about-us/our-funders/

Toutes les opinions exprimées dans cette publication sont celles des auteurs. Elles ne représentent pas forcément la position officielle du CIFOR, des éditeurs, des relecteurs, des institutions auxquelles appartiennent les auteurs ou des sponsors de cette publication. 


\section{Table des matières}

Avant-propos vi vi

1 Présentation de la démarche 1

1.1 Objet du guide 1

1.2 Qu'est-ce que l'élaboration de scénarios ? 2

1.3 Analyse prospective participative 3

En bref $\quad 5$

2 La phase de préparation $\quad 6$

2.1 Impliquer les parties prenantes $\quad 7$

2.2 Mettre en place la logistique et l'organisation 14

3 Mise en œuvre de la PPA $\quad 20$

3.1 Étape 1: Définir le système 21

3.2 Étape 2 : Identifier et définir les facteurs de changement 24

3.3 Étape 3 : Identifier et choisir les facteurs moteurs 31

3.4 Étape 4 : Élaborer les scénarios $\quad 52$

3.5 Étape 5 : Élaborer un plan d'action grâce à la prospective 67

Bibliographie $\quad 76$

$\begin{array}{ll}\text { Annexes } & 77\end{array}$

1 Suggestions des participants pour la mise en œuvre de la PPA
sur la sécurité du foncier forestier

2 Glossaire des termes de prospective utilisés dans ce document $\quad 78$ 


\section{Liste de figures, tableaux, et encadrés}

\section{Figures}

1 Les grands principes de la méthode PPA. 4

2 Principes régissant les relations dans le groupe d'experts. 8

3 La pièce doit pouvoir accueillir tous les participants. 17

4 Susciter la compréhension partagée et l'action commune pour l'avenir des réformes du foncier et de la sécurité foncière dans les forêts. 21

5 Exemples de reformulation des facteurs de changement. 27

6 Exemple de cas rencontré lors de l'analyse I/D. 35

7 Feuille de travail exposant l'influence mutuelle des variables (facteurs). 39

8 Résumé de la structure du logiciel. $\quad 40$

9 Aperçu de l'importance des différentes variables. 46

10 Exemple provenant de l'atelier de formation en Ouganda. 49

11 Graphique des influences indirectes. 50

12 Six exemples de scénarios graphiques produits lors des ateliers PPA au Pérou.

13 Les étapes de la démarche rétrospective. 75

\section{Tableaux}

1 La réunion du groupe d'experts pour élaborer des scénarios en bref.

2 Facteurs internes évoqués lors de la formation des animateurs à Bogor (Indonésie).

3 Facteurs externes évoqués lors de la formation des animateurs à Bogor (Indonésie).

4 Variables d'une analyse structurelle.

5 Direct influence versus direct dependence. 48

6 Comparaison entre la force directe et la force directe pondérée. $\quad 48$

7 Comparaison entre la force directe pondérée et la force indirecte pondérée.

8 Les facteurs moteurs au Pérou. 54

9 Guide d'utilisation du logiciel pour évaluer les scénarios 58

10 Avenirs plausibles de la sécurité du foncier forestier selon les scénarios élaborés au cours des ateliers de formation. 


\section{Encadrés}

1 Constitution d'un groupe d'encadrement en Indonésie 7

2 Constitution d'un groupe d'experts en Ouganda 10

3 Constitution d'un groupe d'experts au Pérou 10

4 Constitution d'un groupe d'experts en Indonésie 10

5 Constitution d'un groupe de travail : le cas du Pérou 13

6 Constitution d'un groupe de travail : le cas de l'Indonésie 13

7 Constitution d'un groupe de travail : le cas de l'Ouganda 13

8 Avantages et inconvénients de l'organisation d'une série d'ateliers 14

9 Exemple de mise en ouvre d'ateliers PPA 16

10 Les facteurs de changement dans le cas du Pérou 31

11 Se baser sur la définition et pas sur le nom de la variable : exemple 33

12 Organiser l'analyse des influences mutuelles 34

13 Exemple de regroupement de facteurs en un seul facteur moteur 51

14 Choisir les facteurs : le cas du Pérou 51

15 Le scénario «Moi d'abord » au Pérou 59

16 Élaboration des scénarios 66

17 Comparaison Rétrospective/Planification conventionnelle 74 


\section{Avant-propos}

Les droits des populations qui vivent dans les forêts ou à proximité sont de plus en plus reconnus, comme les avantages auxquels elles ont droit. La réforme du foncier forestier a une incidence sur les droits et les obligations qui se rattachent aux ressources forestières, comme sur les autorités concernées. Cette incidence peut porter sur les dispositions régissant le transfert, en totalité ou en partie, de droits et d'obligations des instances forestières centralisées vers les autorités coutumières, les usagers des ressources locales, les organisations non gouvernementales ou les gouvernements locaux.

On parle de sécurité foncière lorsqu'un individu ou un groupe de personnes a le sentiment que ses droits sur la terre ou sur d'autres ressources sont garantis (Poffenberger 1990). La sécurité des droits dépend de la perception que ces personnes ont de ces droits et de l'expérience qu'elles en ont, mais aussi de leur nature, c'est-à-dire des droits issus des règles coutumières ou bien inscrits dans la loi. Par conséquent, la " sécurité " correspond à la capacité d'un individu à s’approprier des ressources de façon continue, sans qu'il y ait contrainte, différend ou approbation requise de la part d'un tiers, ainsi qu'à la capacité de revendiquer un retour sur investissement dans lesdites ressources (Mwangi et Meizen-Dick 2009, 310).

Le Centre de recherche forestière internationale (CIFOR) a lancé en Indonésie, en Ouganda et au Pérou le projet GCS-Tenure, une étude comparative mondiale sur la réforme du foncier forestier, grâce à un financement de la Commission européenne (CE), du Fonds international de développement agricole (FIDA), ainsi que du Fonds pour l'environnement mondial (FEM) et de l'Organisation des Nations Unies pour l'alimentation et l'agriculture. Ce projet a vocation à comparer le régime foncier coutumier et celui qui est inscrit dans la loi, ainsi qu'à analyser l'incidence de leur rapport sur la sécurité foncière des communautés qui dépendent des forêts, notamment des femmes et autres groupes marginalisés.

En procédant à des comparaisons à l'échelle mondiale grâce à des méthodologies standardisées, cette étude analyse les différences quant à la réussite ou à l'échec des innovations destinées à renforcer la sécurité des droits fonciers, qu'elles soient institutionnelles ou concernent les politiques publiques. Elle permet aussi d'examiner comment ces innovations déterminent les stratégies susceptibles de conduire aux résultats désirés. Lors de la première étape, les principales parties prenantes ont été mobilisées grâce à l'analyse prospective participative (PPA) (Bourgeois et Jésus 2004). Précédemment utilisée dans d'autres pays, dont l'Indonésie, l'analyse prospective participative est un moyen qui permet d'impliquer les parties prenantes locales dans la mise en œuvre d'un projet et de leur donner la parole. 
En suscitant le consensus, une PPA avec des scénarios facilite la négociation et améliore la pertinence des résultats (Liswanti 2012 ; Shantiko 2012 ; Shantiko et al. sous presse).

Le processus fait participer les parties concernées : fonctionnaires du domaine des forêts (et autres représentants des autorités concernées), représentants des ONG, universitaires, praticiens, secteur privé, etc. Lors de réunions participatives, tous les experts des parties prenantes définissent et élaborent progressivement un éventail de scénarios de sécurité foncière. Ils conçoivent ensuite les actions correspondant aux scénarios définis. Fondée sur les données scientifiques issues d'une étude de cas précédente en Indonésie, cette démarche a permis de renforcer la capacité des parties prenantes en matière de travail en commun d'analyse et de résolution de problèmes.

En appliquant la PPA, l'équipe de chercheurs vise à répondre aux questions suivantes : Quels sont les principaux facteurs qui influencent la sécurité du foncier forestier ? Quelles sont les actions possibles pour atténuer les conséquences néfastes (ou aplanir les obstacles qui freinent la mise en œuvre d'un nouveau régime) et pour favoriser un changement positif (p. ex., un accès équitable pour les femmes et les groupes marginalisés) ? Qui doit être responsable de ces actions ?

L'analyse prospective participative est destinée à aider les instances de décision à comprendre les facteurs clés, les enjeux et les conséquences futures des choix stratégiques. C'est ainsi qu'elles pourront réformer de manière plus performante le régime foncier à divers niveaux, local, régional et national. Le recours aux scénarios permet aussi lors des échanges d'évoquer les ambitions et les projets des personnes, ainsi que la façon dont elles envisagent le changement. Cela leur permet de prendre des décisions aujourd'hui en fonction de changements susceptibles d'arriver à l'avenir* (Wollenberg et al. 2000).

L'analyse prospective participative s'articule autour d'échanges intenses entre les parties prenantes. Elle permet d'aboutir à une compréhension commune de la situation actuelle et de l'évolution du problème, et aussi de parvenir à un accord collectif sur la planification et la gestion des ressources.

La PPA est très couramment appliquée à différents problèmes relatifs à la gestion des ressources naturelles. En Indonésie, grâce à cette méthode, il a été possible d'améliorer les politiques publiques et de régler des questions sociales, surtout quand les outils sont intégrés à la prise de décisions au niveau local (Liswanti 2012). Elle a aussi permis aux instances de décision de se rendre compte qu'il fallait prendre des mesures pour concilier des intérêts contradictoires sur la gestion des ressources naturelles au niveau du district (Shantiko 2012). 



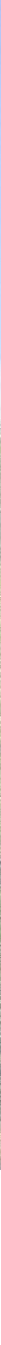

\section{Présentation de la démarche}

Ce chapitre présente l'objet de ce guide (Section 1.1), décrit la coélaboration de scénarios (Section 1.2) et expose les éléments fondamentaux de l'analyse prospective participative (Section 1.3). Il introduit les chapitres 2 et 3 qui expliquent la démarche en détail.

\subsection{Objet du guide}

Ce guide vise à aider les praticiens de terrain à coélaborer au niveau local des scénarios* sur la sécurité du foncier forestier avec un certain nombre de parties prenantes. C'est le produit de l'application de la méthode de l'analyse prospective participative (Bourgeois et Jésus 2004) lors de trois ateliers de formation, de cinq jours chacun. Ceux-ci se sont déroulés en Indonésie, en Ouganda et au Pérou dans le cadre de l'étude comparative mondiale sur la sécurité du foncier forestier réalisée par le CIFOR (appelée projet GCSTenure). Ces trois ateliers ont permis de former une soixantaine de personnes comme animateurs grâce à un processus d'apprentissage par la pratique. 
Ce guide comporte des informations relatives à la préparation, à l'organisation et à la mise en œuvre techniques. Il bénéficie des commentaires des participants des ateliers qui ont évalué la formation et émis des avis utiles et pratiques. La rédaction de ce guide a également été alimentée par les remarques formulées judicieusement par plus de 200 personnes ayant pris part aux dix ateliers de mise en ouvre qui ont eu lieu dans les pays mentionnés.

Il est destiné à apporter aux praticiens les connaissances nécessaires pour comprendre les futurs besoins en matière de réforme et de sécurité foncières et aussi à leur permettre de prendre des mesures ensemble. Les auteurs conseillent aux usagers de répéter les diverses étapes avant de les appliquer avec les parties prenantes sur le terrain.

La sécurité du foncier foresti er est un domaine où règne une grande incertitude*. À l'instar de beaucoup d'autres problèmes de développement, elle dépend fortement de facteurs sociaux, politiques, culturels et économiques. La plupart de ces facteurs sont généralement considérés inhérents à chaque territoire, sans présenter d'évolution rapide ni importante. Cet angle de vue conduit souvent les communautés locales à se sentir dépossédées de tout moyen d'action et à être passives, même lorsqu'il existe des pistes de changement. L'établissement de scénarios participatifs confère une perspective à long terme, ce qui offre aux acteurs locaux la possibilité de devenir agents de changement. En partant de l'avenir pour changer le présent afin d'agir sur la situation future, les collectivités et les parties prenantes locales peuvent proactivement s'engager pour transformer l'avenir de la sécurité du foncier forestier à leur niveau.

Le chapitre 2 énonce des recommandations sur la préparation d'un processus d'élaboration de divers scénarios sur la sécurité du foncier forestier à l'aide de l'analyse prospective participative. Les citoyens travaillent ensemble à la préparation de ce processus. Le chapitre 3 présente cette analyse, étape par étape. Chaque étape aboutit à des résultats qui alimenteront les étapes suivantes. Aucune étape ne peut être négligée sans mettre en péril la cohérence et la logique de la méthode. Chaque étape est documentée par des exemples issus des ateliers de formation mentionnés plus haut et de la mise en ouvre sur le terrain. Elle s'accompagne de conseils utiles émanant des praticiens de terrain ainsi que des commentaires émis par les participants.

Note : Les termes essentiels sont expliqués dans le glossaire en annexe 2. Lors de leur première occurrence dans ce document, ils sont suivis d'un astérisque $\left(^{*}\right)$.

\subsection{Qu'est-ce que l'élaboration de scénarios ?}

La construction de scénarios pour avoir une vision* de l'avenir n'est pas une idée nouvelle. Dans ce guide, «scénario*» désigne la description d'une possible situation future en fonction d'un ensemble explicite, cohérent et logique d'hypothèses sur les relations essentielles entre les composantes concernées et les facteurs à l'œuvre. L'élaboration de scénarios sur l'avenir de la sécurité du foncier forestier vise à explorer 
des transformations plausibles* en identifiant les facteurs et les rapports qui existent entre eux.

Lorsque les scénarios sont destinés à être utilisés au niveau local, la participation des parties concernées est fondamentale. Cependant, le terme même de participation peut être compris et interprété de différentes façons. La participation peut correspondre à la simple consultation de la population locale pour valider les scénarios établis par des équipes externes. Elle peut aussi désigner l'implication des parties prenantes locales dans le processus d'élaboration des scénarios (p. ex., pour recueillir leur avis sur la situation existante). La coélaboration renvoie ici à l'implication constante et totale des parties prenantes locales à toutes les étapes du processus de construction des scénarios, lequel processus dépend des données de départ fournies par ces parties.

La coélaboration est au cœur d'un processus d'autonomisation basé sur le futur (Bourgeois et al. 2017). Il permet aux participants de gagner en confiance à l'égard de leur propre capacité à comprendre leur propre avenir et à l'améliorer. Il crée aussi un fort sentiment d'adhésion, qui est capital pour lancer des actions concernant l'avenir.

Toutes les étapes de l'analyse prospective participative présentées au chapitre 2 sont destinées à susciter une démarche de coélaboration permanente.

\subsection{Analyse prospective participative}

Lanalyse prospective participative (PPA), qui est l'une des méthodes de la prospective*, est utilisée pour étudier l'avenir de la sécurité du foncier fore stier. Elle considère les différents futurs possibles et la conception des actions correspondantes pour atteindre le but souhaité. En d'autres termes, elle facilite la compréhension partagée et les actions communes pour orienter l'avenir sur le plan de la sécurité et des réformes foncières.

La PPA appliquée à la sécurité du foncier forestier sonde l'avenir pour éclairer le présent. Cette approche peut prendre en compte plusieurs axes et leurs relations pour les transformer en un éventail de choix et d'alternatives. Elle permet d'obtenir des connaissances préalables*, c'est-à-dire des connaissances sur les différentes formes d'avenir possibles, les conditions et les raisons pouvant y conduire. Ces connaissances préalables sont utiles à deux points de vue : d'une part, de façon préactive (comment se préparer au mieux si tel ou tel scénario se produit ?) et, d'autre part, de façon proactive (comment influer sur l'avenir afin d'aboutir à la situation souhaitée ?)

La PPA présente des avantages secondaires très intéressants, notamment une meilleure compréhension des facteurs qui agissent sur la sécurité foncière et des liens qui existent entre eux. Elle met au jour les divers éléments cachés derrière l'enjeu de la sécurité du foncier forestier et que les parties prenantes ne cernent pas toujours aisément. Elle fournit aussi une structure claire pour élaborer les scénarios et identifier 


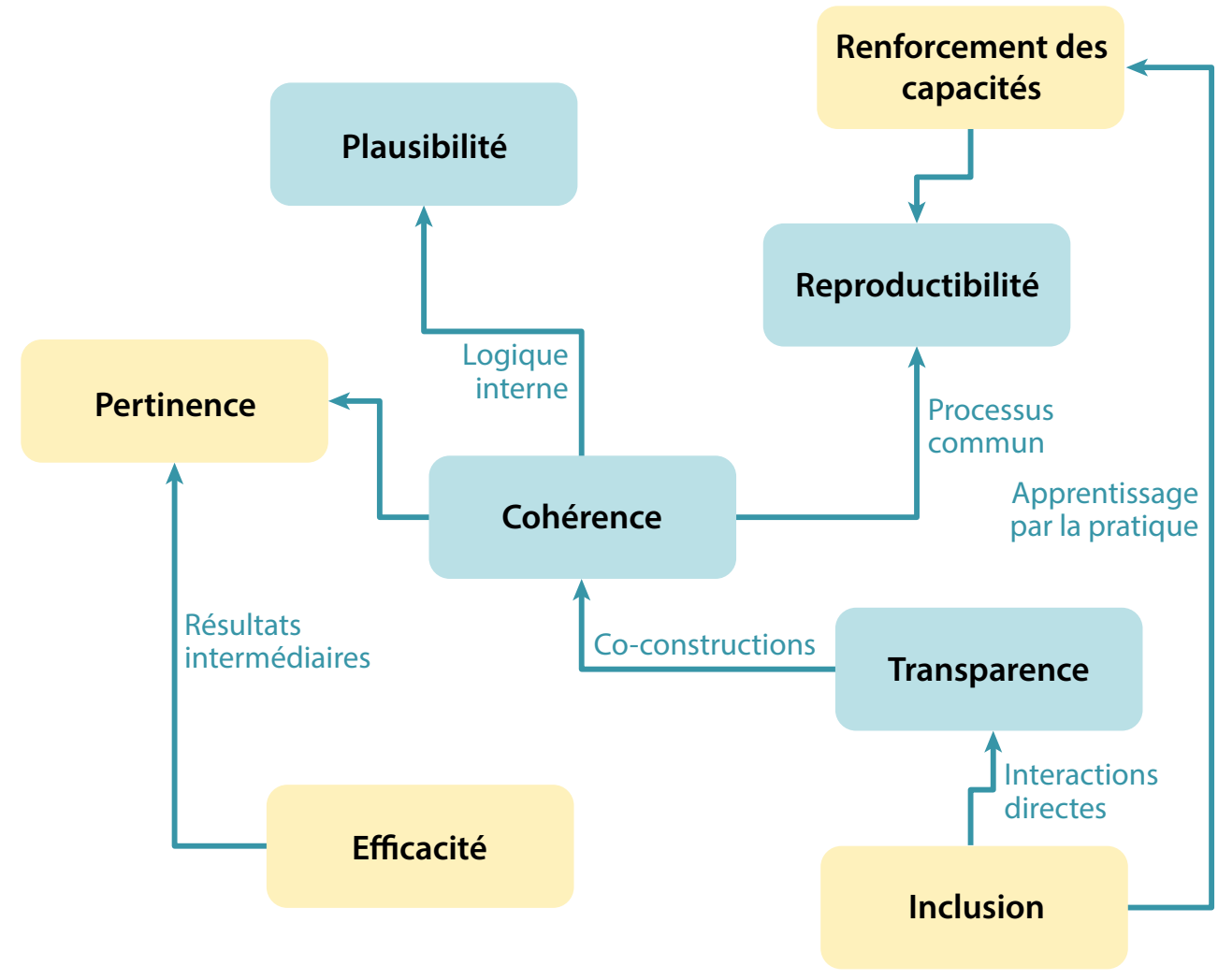

Figure 1. Les grands principes de la méthode PPA.

les facteurs essentiels qui permettront de mettre en place un avenir meilleur pour la sécurité foncière. Le débat sur les moteurs (ou facteurs) du changement est essentiel pour comprendre la problématique du régime foncier et de la sécurité foncière à divers niveaux.

La démarche PPA s'appuie sur deux catégories de principes (voir aussi la figure 1) qui correspondent aux principaux objectifs et aux grandes caractéristiques du processus et des résultats visés :

\subsubsection{Principes relatifs aux objectifs de la PPA}

- Efficacité. Obtenir des résultats dans un délai assez court. Pour l'organisation, il y a plusieurs solutions, mais deux choix principaux : soit un atelier sur cinq jours avec deux semaines de préparation et une à deux semaines pour la finalisation des résultats, soit un processus de quatre à cinq mois divisé en plusieurs points d'étape. 
- Inclusion. Prise en compte des connaissances des parties prenantes dans un cadre de référence exhaustif pour réfléchir à l'avenir. Cette méthode donne suffisamment de temps aux participants pour interagir. Pour les séances de réflexion, des techniques participatives permettent à tous d'exprimer leurs idées et de les voir prises en considération.

- Pertinence. Production de résultats pouvant être utiles pour l'action. La PPA est une méthode appliquée qui est destinée à livrer à l'usager une valeur ajoutée, un avantage direct issu de sa mise en ouvre.

- Renforcement des capacités. Il s'agit d'engager les participants non seulement dans une réflexion sur l'avenir, mais aussi dans le renforcement de leurs capacités grâce à un processus d'apprentissage par la pratique.

\subsubsection{Principes relatifs aux caractéristiques des résultats}

- Cohérence. Logique dans les résultats. Cette cohérence est assurée en suivant rigoureusement une suite d'étapes, chacune produisant des résultats qui viennent alimenter l'étape suivante.

- Reproductibilité. La méthode et le processus peuvent s'appliquer à d'autres sujets et d'autres contextes.

- Transparence. "Subjectivité transparente ", c'est-à-dire qu'il n’y a aucune hypothèse cachée ni falsification de résultats. Toutes les étapes sont clairement et systématiquement documentées et tous les résultats sont mis à disposition de tous les participants et du public.

- Plausibilité. Les scénarios formulés sont jugés raisonnables à cause des hypothèses de départ, de leur cohérence interne et du lien logique avec la réalité. Ce principe est étroitement lié au principe de cohérence.

\section{En bref}

\section{Analyse prospective participative}

L'analyse prospective participative consiste en un cadre de travail comportant plusieurs étapes qui sert à anticiper les changements dans les environnements instables avec le concours d'un certain nombre de parties prenantes. Elle permet de préparer ces parties prenantes à faire face à des évolutions très brusques et de mieux débattre des choix stratégiques. Elle renforce aussi la capacité à produire et à partager des informations utiles pour une prise de décisions efficace. 

pas sur des résultats satisfaisants et de qualité. Par ailleurs, les participants subissant une pression supérieure à ce qu'ils peuvent supporter n'adhéreront pas à la démarche proposée. La qualité et la pertinence ne doivent pas être sacrifiées pour raccourcir les délais.

\subsection{Impliquer les parties prenantes}

Trois groupes différents d'acteurs doivent participer à la mise en ouvre d'un processus de coélaboration, comme la méthode PPA, dans le domaine de la sécurité du foncier forestier : un groupe doté de responsabilités (ci-après désigné le "groupe d'encadrement "), un groupe d'intervenants directement impliqués dans le processus de coélaboration (ciaprès désigné le " groupe d'experts* »), et un groupe chargé de la mise en ouvre technique (ci-après désigné le « groupe de travail »).

\subsubsection{Constituer un groupe d'encadrement : du soutien à l'adhésion}

La principale difficulté de tout travail de prospective est d'éclairer l'action publique au-delà du cercle des intervenants (le groupe d'experts). Dans cette optique, le groupe d'encadrement veille ce que les principales parties prenantes soutiennent le processus de mise en œuvre et ne s'y opposent pas. Elles seront ainsi plus enclines à s'approprier les résultats et à les traduire en actes.

Le groupe d'encadrement est composé de hauts fonctionnaires ou de représentants des pouvoirs publics et d'organisations. Celles-ci peuvent être des organisations de la société civile (OSC) et des chefs
Encadré 1. Constitution d'un groupe d'encadrement en Indonésie

En Indonésie, un atelier régional s'est tenu à Lampung et à Maluku sur les premières phases du projet GCS-Tenure. Dans le cadre d'une réforme du foncier forestier en Indonésie, c'est à l'Agence des forêts, au niveau des provinces et des districts, qu'il incombe de mettre en œuvre les différents programmes de réforme. Des formateurs ont présenté cette démarche aux directeurs de I'Agence des forêts à Lampung et à Makula, de même au niveau des provinces et des districts.

Ces organismes publics se sont engagés dans une démarche PPA depuis le début. Ils ont aidé le formateur à choisir les membres du groupe d'experts qui ont été impliqués dans la mise en œuvre des réformes touchant les forêts. Ils ont aussi donné leur avis et formulé des propositions à la fin de chaque atelier PPA. Ce groupe d'encadrement s'appelle aussi « comité de pilotage ». Tous les membres du groupe d'experts ont réagi positivement à l'implication du groupe d'encadrement, qui est également instance de décision, dans le processus. Le groupe d'encadrement est censé mettre en œuvre le plan d'action prévu par le groupe d'experts au cours de la démarche PPA. 
coutumiers concernés par la sécurité du foncier forestier au sens large. Voici quelques critères de sélection des membres du groupe d'encadrement :

- Qui a les moyens ou le pouvoir d'encourager/de bloquer le processus de mise en œuvre?

- Qui doit s'approprier les résultats pour potentiellement faire évoluer la situation ?

- Qui a la capacité de traduire les résultats en actes ?

Qui a le pouvoir d'affecter ou non du personnel au groupe d'experts?

Les réponses à ces questions détermineront la composition du groupe d'encadrement. Chaque membre pressenti doit être approché individuellement, mais aussi par l'intermédiaire de sa hiérarchie avec l'objectif de l'informer et de l'inviter à prendre part à l'initiative. Le groupe d'encadrement doit aussi être tenu informé des progrès au fur et à mesure des étapes. Il peut émettre des commentaires et des suggestions, mais n’est pas autorisé à modifier les résultats présentés par le groupe d'experts.

Il revient aux membres du groupe d'encadrement de diffuser largement les résultats et de prendre des mesures relatives aux décisions, pour passer progressivement du soutien à l'adhésion.

\subsubsection{Constitution du groupe d'experts : de l'expertise individuelle aux connaissances collectives}

Le groupe d'experts émet des avis techniques lors de la démarche prospective et se mobilise tout au long du déroulement des travaux. Le groupe d'experts est composé de personnes issues de différents milieux et dotées d'expériences diverses. Le terme d'expert désigne ici une personne riche de compétences, de connaissances, de discernement ou d'un savoir-faire dans un domaine particulier grâce à la recherche scientifique, à son expérience, à sa perspicacité ou à son métier. Les réunions du groupe d'expert sont un espace où les quatre principes, exposés à la figure 2 , déterminent les échanges.

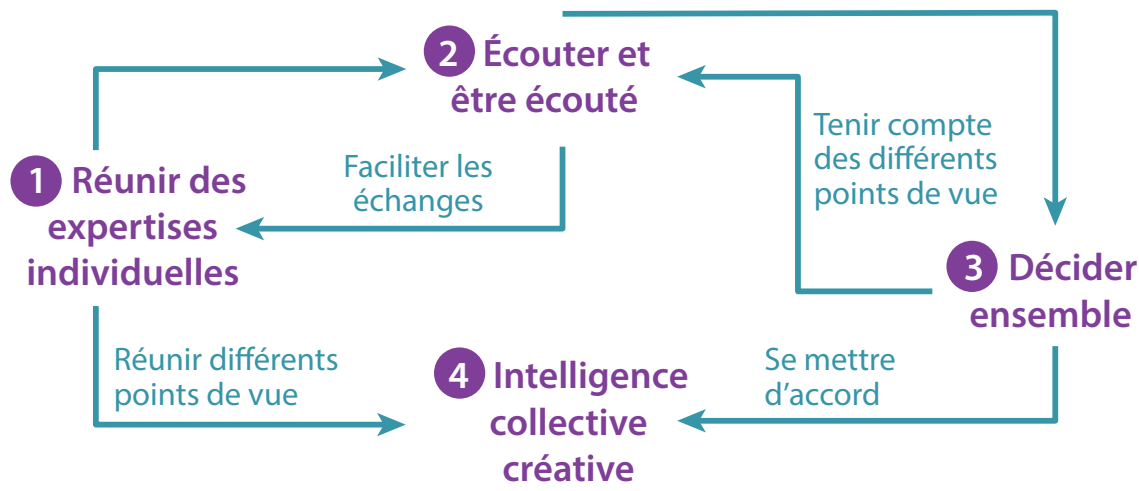

Figure 2. Principes régissant les relations dans le groupe d'experts. 
Pour que le groupe d'experts fonctionne bien, il convient de choisir ses membres avec soin. Voici les critères proposés pour le choix de ses membres :

- Connaissances. En réunissant suffisamment de participants divers, la démarche PPA peut tenir compte de toutes les dimensions d'un sujet. Ici, par connaissances, nous entendons la formation initiale et complémentaire, mais aussi l'expérience pratique. Un agriculteur, un professeur, un juriste, un commerçant ou un chef de village sont tous experts dans leur domaine : l'agriculture, l'enseignement, le droit et la réglementation, le marché et l'administration locale.

- Diversité socioéconomique. Il convient de veiller à un juste équilibre entre les sexes, les âges, et, le cas échéant, la diversité ethnique et le pouvoir économique. Les groupes d'experts ne doivent pas être composés à partir de l'élite. Les participants doivent au contraire refléter la diversité du territoire et de la population dont le futur est étudié de près.

- Caractéristiques individuelles. Les experts doivent être sélectionnés pour leur ouverture d'esprit dans le débat et leur tolérance vis-à-vis d'autres opinions. Les personnes étroites d'esprit doivent être exclues, même si elles ont de bonnes connaissances, car elles sont susceptibles d'entraver le travail du groupe ou de nuire à son objectivité.

- Inclusion. L'éducation, la maîtrise d'une langue précise (c.-à-d. parler, lire et écrire) et les autres différences culturelles ne sauraient constituer des critères de sélection. Sinon il y a le risque qu'aucun participant ne connaisse la situation de certains éléments précis du système*. En cas de besoin, les participants devront être aidés pour lire, écrire ou s'exprimer à l'oral dans une autre langue que la leur, afin de pouvoir pleinement s'impliquer dans les débats. Il faut le cas échéant prévoir une personne pour noter leurs idées sur des fiches ou lire tout haut les informations à débattre.

- Disponibilité. Tous les experts qui prennent part au premier atelier doivent être présents à tous les autres. Étant donné qu'ils s'engagent dans un processus d'élaboration de scénarios, ils ne peuvent pas manquer une réunion ou se faire remplacer par n'importe qui. Les participants doivent être d'accord sur cette règle dès le départ. L'arrivée d'un nouveau participant ou l'absence d'un membre lors d'une étape doivent rester exceptionnelles et ne pas mettre en péril les travaux du groupe.

- Expertise individuelle. Les participants ne sont pas sélectionnés en raison de leur appartenance à une organisation ou à un secteur spécifiques. Ils sont choisis pour un savoir pouvant permettre de mieux appréhender le futur de la sécurité du foncier forestier. Dès qu'ils sont invités à faire partie du groupe d'experts, on leur demande d'oublier leur fonction ou poste officiels pour se considérer mutuellement comme de simples participants.

Pour constituer un groupe d'experts, il faut dresser la liste des participants les plus évidents. Il s'agit de personnes connues et qui répondent aux critères énumérés cidessus. Quand cette première liste est établie, les personnes y figurant peuvent aider à en trouver d'autres, qui seront contactées et invitées selon les mêmes critères. 
Encadré 2. Constitution d'un groupe d'experts en Ouganda

En Ouganda, l'équipe du projet avec les comités de pilotage au niveau de trois districts ont constitué un groupe d'experts. Les critères de sélection étaient :

- connaissances/expérience/ intérêt pour les questions foncières, notamment dans les forêts ;

- être disponible le temps des deux ou trois jours d'ateliers PPA.

En raison de ces deux critères, de nombreuses femmes ont été mises à l'écart d'office puisque, en Ouganda, ce sont les hommes qui s'occupent des questions foncières, notamment dans les forêts. Dans une optique d'égalité entre les sexes, des ateliers PPA destinés uniquement aux femmes ont été organisés dans chaque district. En plus des critères de sélection évoqués ci-dessus, des chefs politiques et religieux et des leaders sur le plan culturel et socioéconomique ont été recherchés. Au total dans les trois districts, 144 personnes ont participé aux activités PPA, soit 32 femmes et 112 hommes. La moyenne par activité était de 5 femmes et 19 hommes.

Enseignement Les critères de sélection doivent permettre l'inclusivité des femmes.

\section{Encadré 3. Constitution d'un groupe d'experts au Pérou}

Dans la région de Madre de Dios, 10 personnes ont participé au premier atelier et 15 au second. Dans la région de Loreto, 30 personnes ont participé au premier atelier et 25 au second.

Enseignements de I'application de la PPA dans deux régions amazoniennes au Pérou:

- Diversité des experts : faire appel à des personnes dans diverses disciplines (gestion forestière, foresterie communautaire, éducation rurale, questions de santé, conservationa, délivrance de titres de propriété, droits des communautés, budget du gouvernement, projets productifs, professeurs d'université, membres des communautés).

- Débat ouvert: afin de rassembler le plus d'informations disponibles.

- Rencontrer très régulièrement les experts: pour faire les bons choix et pour que les experts répondent aux attentes avant, pendant et après I'atelier.

- La qualité prime sur la quantité : pour disposer d'experts souhaitant contribuer au débat.

\section{Encadré 4. Constitution d'un groupe d'experts en Indonésie}

Contexte de la PPA à Lampung et à Maluku. À Makulu, où la coutume est vivace, la plupart des communautés traditionnelles possèdent un vaste territoire forestier coutumier ; cependant, la réforme forestière n'a pas encore démarré. À Lampung, les migrants sont beaucoup plus nombreux. Cela fait longtemps que le régime foncier pose problème et que la forêt a cédé la place aux cultures de rapport ; des réformes du régime foncier ont été mises en œuvre avec succès en certains endroits.

Le choix des experts sur chacun de ces sites présentait donc un défi différent. Le groupe d'experts devait être composé de personnes de différents milieux et dotées d'expertises variées (délivrance de titres de propriété, foresterie communautaire, chef coutumier, université, gouvernements, ONG et secteur privé). II était souhaitable que ses membres soient ouverts d'esprit, désireux d'écouter, tolérants, actifs dans le processus, ni dominants ni égoïstes, respectueux des opinions d'autrui.

Les ateliers de lancement à Lampung et à Maluku ont permis de repérer des candidats potentiels en Indonésie grâce à la procédure de détermination des parties prenantes. La liste en a été transmise au groupe d'encadrement qui a aidé à sélectionner les participants. À Maluku, les trois ateliers PPA ont été suivis respectivement par 19,14 et 17 personnes. Les deux ateliers de Lampung ont réuni 19 et 17 personnes. 
Le nombre de membres approprié n'est pas défini à l'avance. S'il y a trop de participants, la méthode ne fonctionnera pas et le processus sera moins interactif. S'il y en a trop peu, les résultats seront moins pertinents et des biais seront introduits. D'après l'expérience, une vingtaine de personnes convient habituellement pour traiter des diverses composantes de la sécurité foncière et garantir de bonnes conditions de travail pour la réussite de la coélaboration. La mise en œuvre au Pérou a permis de s'apercevoir que la qualité des participants était plus importante que leur nombre. L'équipe du Pérou conseille de sélectionner les participants avec soin. Elle les a rencontrés plus d'une fois pour s'assurer de faire le bon choix.

Intégrer des personnes ayant des opinions différentes (p. ex., des parties prenantes ayant un point de vue plus ou moins inclusif sur le rôle des communautés dans les forêts). Dans le cas du Pérou, presque tous les participants s'accordaient sur le fait que les communautés devraient avoir accès aux forêts en affirmant qu'elles les protègent traditionnellement. Il a été noté avec intérêt qu'un certain nombre de participants considéraient que des règles plus sévères étaient nécessaires pour éviter d'aggraver la déforestation. Les conclusions de l'atelier ont permis de résoudre certains problèmes fonciers de la communauté, mais elles doivent représenter plus d'un seul angle de vue. Certains membres défendent l'intégrité des forêts, d'autres des intérêts privés, d'autres encore le gouvernement, etc.

\subsubsection{Constitution d'un groupe de travail : De l'animation à la coélaboration}

Pour appliquer la PPA à la sécurité du foncier forestier, il est nécessaire de faire appel à des personnes qualifiées. À cette fin, les trois ateliers en Indonésie, en Ouganda et au Pérou ont permis de former des animateurs pour le projet GCS. 
Au cours d'une session d'apprentissage par la pratique qui a duré cinq jours, les participants ont été formés à la PPA, ainsi qu'à des techniques d'animation spécifiques tout en renforçant leurs compétences relationnelles. Lobjectif était de leur fournir les connaissances pratiques et le savoir nécessaire dans le cadre de la coélaboration de scénarios pour l'avenir de la sécurité du foncier forestier au niveau local.

Pour diriger l'atelier, il faut au moins deux animateurs formés et neutres, n’appartenant pas aux parties prenantes. Leur rôle est le suivant :

- diriger et gérer la session étape par étape sans en modifier le contenu ;

- veiller à ce que tous les participants aient la même possibilité d'intervenir ;

- $\quad$ suivre toutes les étapes et appliquer les règles convenues ;

- parvenir à un accord avant de passer à l'étape suivante ;

- conserver une trace des décisions et des résultats intermédiaires ;

- gérer éventuellement les partis pris et les personnes qui accaparent le débat ; ceci devrait être évité par un choix judicieux des participants à qui les règles seront expliquées avec précision.

Le processus vise à transformer progressivement la dynamique pour passer de la réflexion avec les animateurs à la coélaboration. L’expérience montre que les participants sont souvent très volontaires et aptes à s'impliquer plus directement dans les différentes phases de travail. Les animateurs doivent surtout veiller à ce que les éléments énumérés ci-dessus restent au centre de la dynamique de travail.

Compte tenu de l'abondance des informations produites, les deux animateurs devraient être aidés par d'autres intervenants, pour composer un vrai groupe de travail.

Les animateurs et certains intervenants doivent être capables de se servir d'un ordinateur portable sur lequel est enregistré le logiciel de l'analyse structurelle et de l'analyse d'incompatibilité. Il est nécessaire qu'ils sachent utiliser des logiciels de base comme un outil de traitement de texte, un tableur et un logiciel de création de diapositives (facultatif). 


\section{Encadré 5. Constitution d'un groupe de travail : le cas du Pérou}

Au Pérou, nous avions une équipe d'au moins trois personnes :

- un animateur impliqué dans toutes les activités du projet de recherche et qui connaissait les principaux enjeux de la réforme foncière du pays ;

- un animateur qui coordonnait la méthodologie PPA et participait à tous les ateliers ;

- un animateur par région qui connaissait le contexte local et régional et rencontrait très régulièrement le groupe d'experts et le groupe d'encadrement.

\section{Enseignements La} coordination entre les membres du groupe de travail est déterminante pour la réussite de l'atelier. Les bonnes relations permettent de mieux respecter le calendrier et améliorent le dialogue avec les experts.
Encadré 6. Constitution d'un groupe de travail : le cas de l'Indonésie

En Indonésie, deux membres du projet GCS-Tenure ont animé les ateliers PPA à Lampung. À Maluku, les ateliers ont été animés par trois personnes (une du projet GCS-Tenure et deux travaillant à Maluku chez un de nos partenaires dans le projet). Tous les animateurs avaient été formés à Bogor avant le démarrage des ateliers. Ils savaient comment appliquer la PPA dans le contexte d'une réforme du foncier forestier (principaux enjeux, contraintes et difficultés) ; ils étaient rompus aux activités des projets de recherche dans différentes régions et veillaient à l'équité entre les participants durant le processus. Ils n'influençaient pas les participants et ne leur fournissaient pas les réponses. Ils étaient attentifs à faire participer toutes les personnes présentes. De temps à autre, ils encourageaient les participants à animer certaines étapes du processus pour leur offrir une expérience nouvelle.
Encadré 7. Constitution d'un groupe de travail : le cas de l'Ouganda

En Ouganda, les quatre animateurs de l'équipe (trois femmes et un homme) ont été formés lors de séances préalables. Cependant, dans chaque district étudié, deux autres personnes ont reçu une formation et certains habitants sont venus aider, surtout pour la traduction (différentes langues locales étant pratiquées dans les trois districts étudiés et certains participants ne comprenant pas l'anglais). Deux animateurs s'assuraient le concours d'un traducteur pour chaque session. Les deux autres animateurs notaient le déroulement du processus. 


\subsection{Mettre en place la logistique et l'organisation}

\subsubsection{Mettre en œuvre les ateliers pour le groupe d'experts}

Option 1 : une séquence de deux ou trois ateliers d'une durée de deux ou trois jours chacun sur une période pouvant aller d'un mois à deux mois. Ainsi, les participants se souviennent encore de ce qu'ils ont fait précédemment. Option $2:$ un atelier de six jours qui regroupe toutes les étapes. Le tableau 1 présente les éléments essentiels d'une réunion d'un groupe d'expert pour élaborer des scénarios.

Ces deux options ont leurs avantages et leurs inconvénients. Notre expérience montre que l'option 1 livre de meilleurs résultats. Dans celle-ci, le groupe d'experts et le groupe de travail disposent de temps pour réfléchir aux résultats obtenus et préparer les données qui serviront à l'étape suivante. L'analyse est plus approfondie. La difficulté est de pouvoir conserver les mêmes personnes dans le groupe d'experts sur une période un peu longue. Cependant, on contourne cela en général en sélectionnant les membres en partie pour leur disponibilité à long terme. Il n'est pas aisé de trouver des personnes qui peuvent s'engager pendant six jours d'affilée et, par définition, l'option 1 évite ce problème.

Lavantage de l'option 2 est de " pouvoir faire le travail » rapidement. Mais les membres du groupe d'experts sont obligés d'être disponibles pendant une semaine complète. L'autre contrainte pour les participants est de parvenir à des résultats le dernier jour. Généralement, cette approche ne permet pas d'élaborer complètement des scénarios ni de s'engager dans un bon plan de travaux grâce à l'analyse rétrospective (processus inversé partant de la définition d'un futur plausible pour déterminer ce qui doit se passer pour que ce futur survienne dans le prolongement du présent). D’autres réunions s'avèrent souvent nécessaires après l'atelier de six jours.

Encadré 8. Avantages et inconvénients de l'organisation d'une série d'ateliers

En Ouganda, au Pérou et en Indonésie, les participants ont considéré qu'il était difficile d'assister à l'atelier plus de deux ou trois jours, surtout les représentants des autorités. Les travaux ont donc été organisés en deux ateliers de trois jours chacun (Lampung et Ouganda), trois ateliers de deux jours (Maluku) et deux ateliers de deux jours (Pérou).
Le personnel du GCS en Indonésie, en Ouganda et au Pérou a préféré une solution intermédiaire avec des organisations différentes (voir les encadrés 5, 6 et 7). Cette solution réduit les contraintes et bénéficie des avantages des deux options :

Le groupe de travail dispose d'un temps suffisant pour évaluer les résultats, préparer le matériel et consulter le groupe d'encadrement. D'un autre côté, cela représente beaucoup de travail et plus de responsabilité pour le groupe de travail. Dans tous les cas, ce groupe reste pratiquement jusqu'à minuit 
Tableau 1. La réunion du groupe d'experts pour élaborer des scénarios en bref.

\begin{tabular}{ll}
\hline Caractéristiques & Besoins \\
\hline Groupe d'experts (20 experts) $^{\text {a }}$ & Accord/implication des experts \\
Équilibre dans le choix des experts $_{\text {Animateurs neutres (groupe de travail) }}$ & Budget pour l'organisation \\
Parvenir à un consensus & Matériel (tableaux, chevalet de conférence, \\
Règle pour prendre les décisions & aimants, etc.) \\
Secrétariat de la réunion & Espace de réunion et de travail avec prise \\
Présence permanente des experts & électrique, ordinateur portable, projecteur \\
& Secrétaire (groupe de travail) \\
\hline
\end{tabular}

a Dans l'idéal, le groupe d'experts comprend 17 à 20 personnes. Cependant, il a été parfois plus important (27 personnes, Loreto au Pérou) ou plus restreint (10 à 15 personnes, Madre de Dios au Pérou)

tous les soirs pour terminer les tâches en cours et disposer des résultats le lendemain. Ces options ont été choisies en raison des contraintes de ressources qui pesaient sur le projet pour la mise en œuvre de la PPA.

La mobilisation de personnes dans un processus conduisant à une compréhension partagée et à une action commune pour la réforme du régime foncier et la sécurité foncière à l'avenir ne se réduit pas à l'utilisation d'outils de prospective. Il s'agit davantage de faire évoluer le point de vue des parties prenantes et de les transformer en actes. Il est donc nécessaire d'allouer des ressources pour le bon déroulement du processus.

Dans tous les cas, avant de démarrer la série d'ateliers, le groupe de travail doit s'assurer de la disponibilité et de l'adhésion des membres du groupe d'experts, ainsi que de l'intérêt et de l'engagement du groupe d'encadrement. Cette vérification est indispensable pour que les membres du groupe d'experts puissent coélaborer les scénarios. Les membres du groupe d'encadrement et du groupe d'experts ayant été choisis, ayant reçu les instructions nécessaires et étant d'accord sur la démarche de mise en ouvre, il convient de planifier avec soin la logistique.

\subsubsection{Matériel nécessaire et organisation pratique}

Les techniques de visualisation permettent aux personnes de participer. On peut utiliser divers supports pour présenter les résultats obtenus lors de chaque étape (panneau de polystyrène, chevalet de conférence, murs, écran) et des fournitures pour présenter les résultats (fiches de couleur, marqueurs, ruban adhésif, aimants, colle repositionnable). 
La salle de réunion doit être équipée d'une source d'électricité, d'au moins un ordinateur portable et, si possible, d'un appareil pour projeter les résultats sur un écran ou sur le mur.

\section{Encadré 9. Exemple de mise en œuvre d'ateliers PPA}

Au Pérou, deux ateliers de deux journées chacun ont été organisés ; en Ouganda, il y a eu deux ateliers de trois jours et en Indonésie trois ateliers de deux jours et deux ateliers de trois jours. L'intensité des travaux dépend des objectifs. Chaque groupe de travail fait des heures supplémentaires pendant l'atelier pour parvenir à son objectif journalier afin de ne pas prendre de retard. II s'engage aussi à participer à la totalité du processus. Si l'atelier se déroule de manière appropriée avec des participants bien choisis, il aboutit aux résultats attendus. Dans le cas de l'Ouganda, afin de pouvoir impliquer les représentants des autorités totalement sans interruption au cours des trois jours, le lieu retenu pour l'atelier se trouvait loin du district ciblé. Cela leur a permis de se concentrer sur l'exercice sans être dérangés par les besoins du service.

En général, toutes les étapes de la PPA peuvent s'articuler ainsi :

Option A : Atelier 1 (Jour 1 - Identifier et définir les facteurs de changement ; Jour 2 - Analyser les variables d'influence). Atelier 2 (Jour 1 - Choisir les facteurs et définir leur configuration, et élaborer les scénarios [continués par le groupe de travail après l'atelier]) ; Jour 2 - Réaliser l'analyse rétrospective et élaborer les plans d'action.

Option B : Atelier 1 (Jour 1 - Définir le système et déterminer les facteurs de changement ; Jour 2 - Définir les facteurs de changement et présenter l'analyse des variables d'influence). Atelier 2 (Jour 1 - Analyser l'influence des variables; Jour 2 - Définir les principaux facteurs). Atelier 3 (Jour 1 - Déterminer la configuration des variables; Jour 2 - Élaborer les scénarios).

Option C : Atelier 1 (Jour 1 - Définir le système et déterminer les facteurs de changement ; Jour 2 - Définir les facteurs de changement et présenter l'analyse des variables d'influence; Jour 3 - Analyser l'influence des variables). Atelier 2 (Jour 1 - Analyser l'influence des variables ; Jour 2 - Définir les principaux facteurs et déterminer la configuration des variables; Jour 3 - Élaborer les scénarios).

\section{Enseignements}

Cette approche peut être adaptée au contexte local. Si l'on impose un processus inapproprié aux membres du groupe d'experts, les résultats finaux de la PPA s'en ressentiront. 
La taille de cette salle doit permettre d'accueillir tous les participants (ou alors prendre des dispositions à cet effet). Ceux-ci doivent pouvoir facilement voir les résultats et échanger entre eux. Une disposition en $U$ répond à ces deux besoins (voir la figure 3)

S'il n'y a pas de tableau blanc, on peut utiliser les murs, surtout s'il n'y a que deux ateliers de deux jours et que le travail de groupe est nécessaire. Le choix du lieu de l'atelier est déterminant. La pièce doit être organisée bien à l'avance.

\section{Ressources financières}

Les participants peuvent être dédommagés en contrepartie de leur présence : frais de déplacement, repas et logement. Les conditions de ce dédommagement doivent être claires pour éviter tout malentendu. Les règles de ce dédommagement seront les mêmes pour tous les participants qui en auraient besoin.

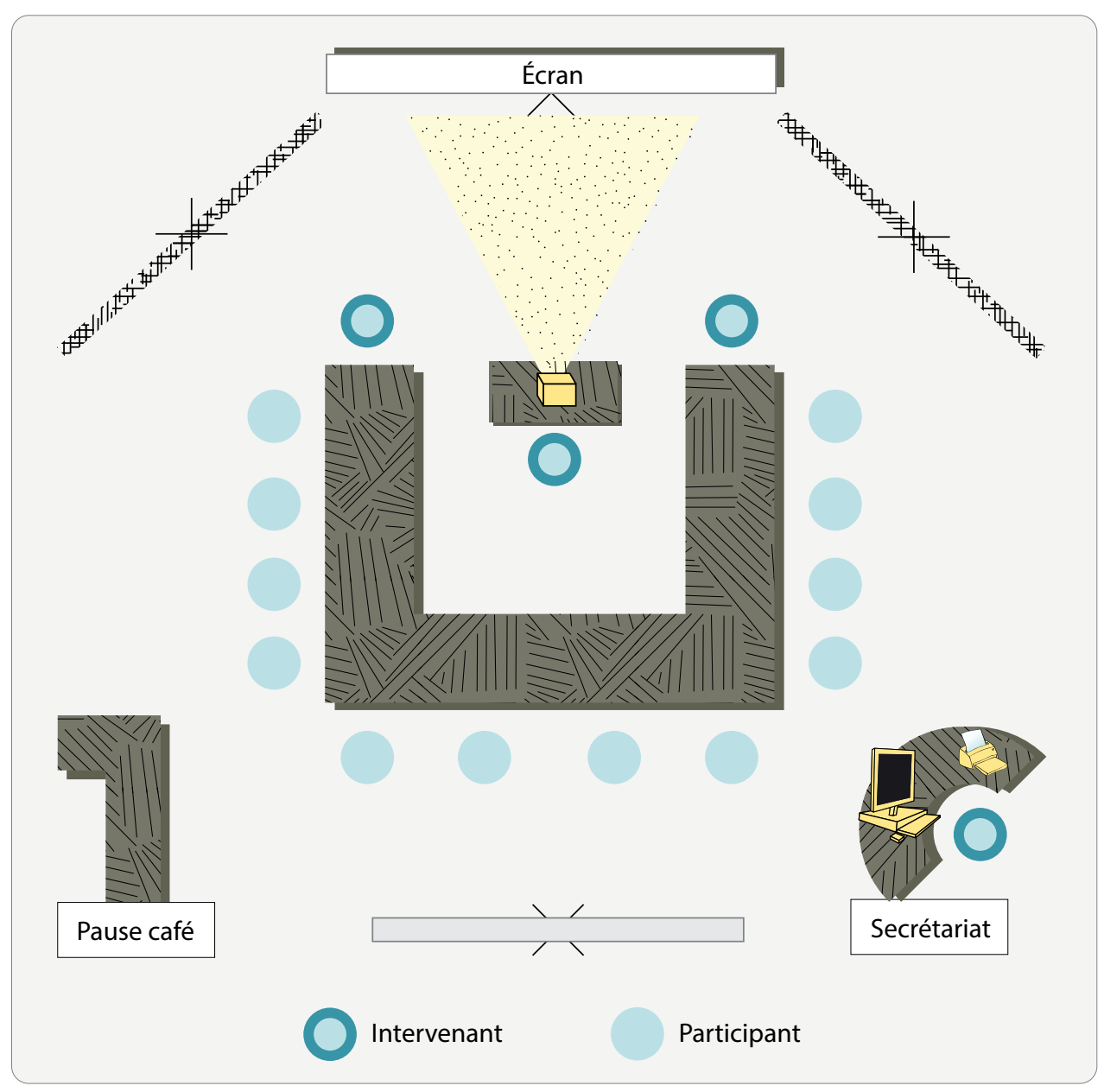

Figure 3. La pièce doit pouvoir accueillir tous les participants. 
Le budget d'organisation d'un atelier sur six jours ou d'une série d'ateliers sur trois jours est variable en fonction du pays. Il dépend du lieu et de la logistique. Cependant, lors de la préparation du budget, il convient de prendre en compte les éléments suivants :

- Penser à un soutien logistique au niveau local. Le travail de l'animateur pendant les ateliers est très intense et nécessite un soutien sur le plan de la logistique et de l'organisation.

- Frais de déplacement de l'équipe d'animateurs.

- Essayer de trouver une imprimante pour l'équipe sur place. Si ce n'est pas possible, veiller à que cette imprimante et/ou ce photocopieur se trouvent près des ateliers.

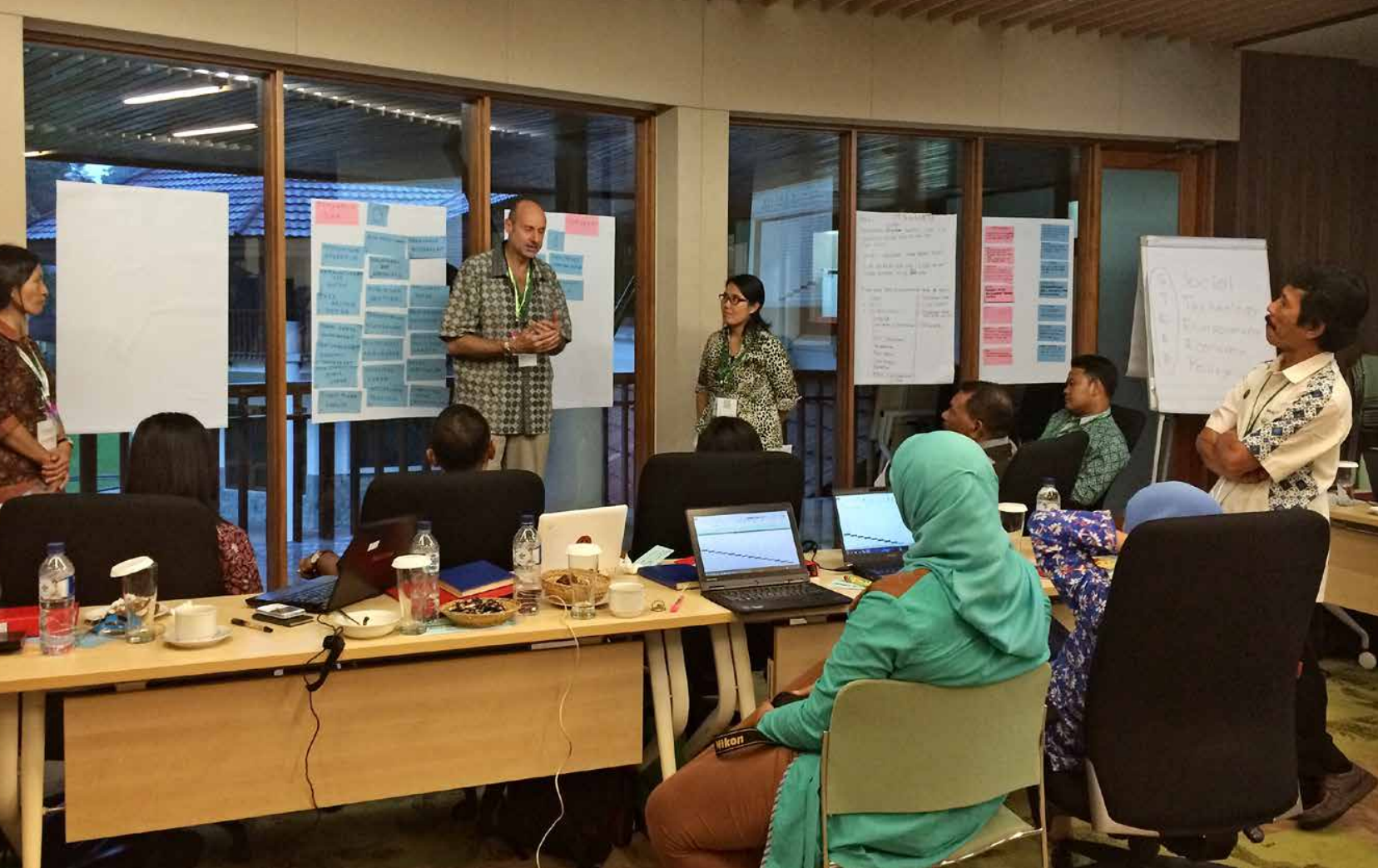





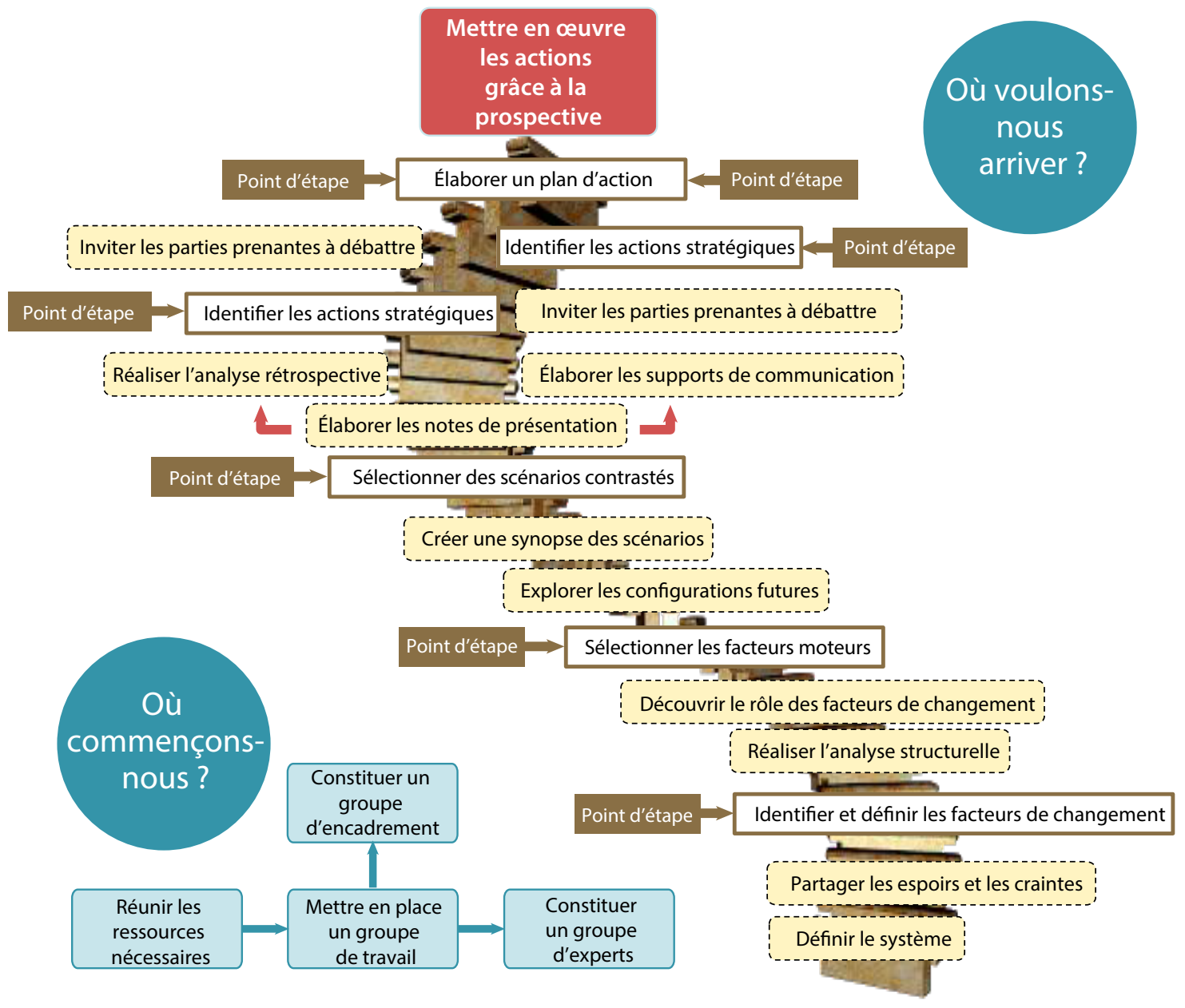

Figure 4. Susciter la compréhension partagée et l'action commune pour l'avenir des réformes du foncier et de la sécurité foncière dans les forêts.

\section{1 Étape 1 : Définir le système}

La première étape du processus est de définir précisément la question à traiter au moyen de la prospective. Il y a quatre composantes dans cette question : objet, lieu, durée et personnes concernées. Ces quatre composantes définissent ce qu'on appelle le « système ». Ce sont les transformations plausibles de ce système qui seront explorées grâce à cette démarche prospective. 


\section{Objet}

La question est la suivante : Quels seraient les différents avenirs possibles de la sécurité du foncier forestier?

\section{Lieu}

- Il convient de définir clairement l'espace géographique concerné. La sécurité du foncier forestier étant liée à un lieu précis, ce type de prospective s’applique en général à un " territoire ». Le territoire correspond à une zone locale (village, district, commune ou petite unité agro-éco-sociologique).

- Le périmètre géographique de la zone de travail doit donc être bien défini. C'est généralement l'objet lui-même qui détermine les limites de la zone.

- Parfois, une unité administrative peut servir à définir la localisation (p. ex., un district dans le cas d'une démarche prospective concernant la planification de l'utilisation des terres en Indonésie).

- Il peut aussi s'agir d'une limite physique (p. ex., l'île de Mayotte ou l'île de Tanimbar en Indonésie) ou d'un écosystème (p. ex., un bassin hydrographique). Il faut savoir que les limites administratives ne sont pas toujours les plus pertinentes pour définir la dimension spatiale d'un système, surtout lorsque les forêts recouvrent plusieurs unités administratives.

\section{Durée}

- Il s'agit à la fois de l'horizon temporel* de la démarche prospective (l'échéance la plus lointaine prévue dans le cadre du projet) et du calendrier* (la totalité de la période considérée).

- Le calendrier peut être envisagé dans une fourchette comprise entre 10 et 50 ans. Une durée inférieure à 10 ans n'est pas suffisante pour explorer les changements significatifs et les ruptures* potentielles. Inversement, une durée supérieure à 25 ans ne favorise pas l'adhésion des acteurs locaux, surtout pour la prise de décisions. Un calendrier s'étalant sur plus de 25 ans est aussi plus abstrait et l'on peut douter de la crédibilité du résultat. Dans le cadre d'une démarche prospective concernant l'avenir de la sécurité du foncier forestier, un calendrier sur 10 à 20 ans est recommandé. La durée dépendra du contexte local (p. ex., envisager une planification nationale à moyen ou long terme pour suivre la sécurité foncière).

- Un calendrier sur 10 à 20 ans correspond aussi à l'arrivée d'une nouvelle génération dont l'avenir préoccupe la génération actuelle.

- Il convient de noter que ce type de réflexion est différent de celui d'une planification stratégique* ou d'une politique dont le calendrier est d'environ cinq ans. 


\section{Personnes concernées}

- Le système est pleinement défini lorsque toutes les parties prenantes sont identifiées. Par parties prenantes, on entend les diverses personnes dont l'avenir est lié à l'avenir du territoire et qui sont affectées par l'objet étudié dans le cadre de la démarche prospective.

- Dans la démarche prospective sur l'avenir de la sécurité du foncier forestier au niveau local, les parties prenantes sont généralement tous les habitants du territoire, ainsi que toutes les personnes qui y travaillent. Dans le cadre du projet GCS-Tenure, les communautés locales doivent aussi être intégrées au processus.

- La liste des parties prenantes doit être bien établie afin de ne pas oublier d'acteurs importants. Les connaissances de ces différents types de parties prenantes sur le territoire doivent se retrouver dans le groupe de personnes qui seront mobilisées pour la mise en œuvre locale du processus d'élaboration des scénarios.

\section{ITADORES (3)}

\section{personas} apacitadas en el uso de la nerodologia - con conocimientos sobte Tenencia de rierras y acooros legales ientrales. ervivido reto

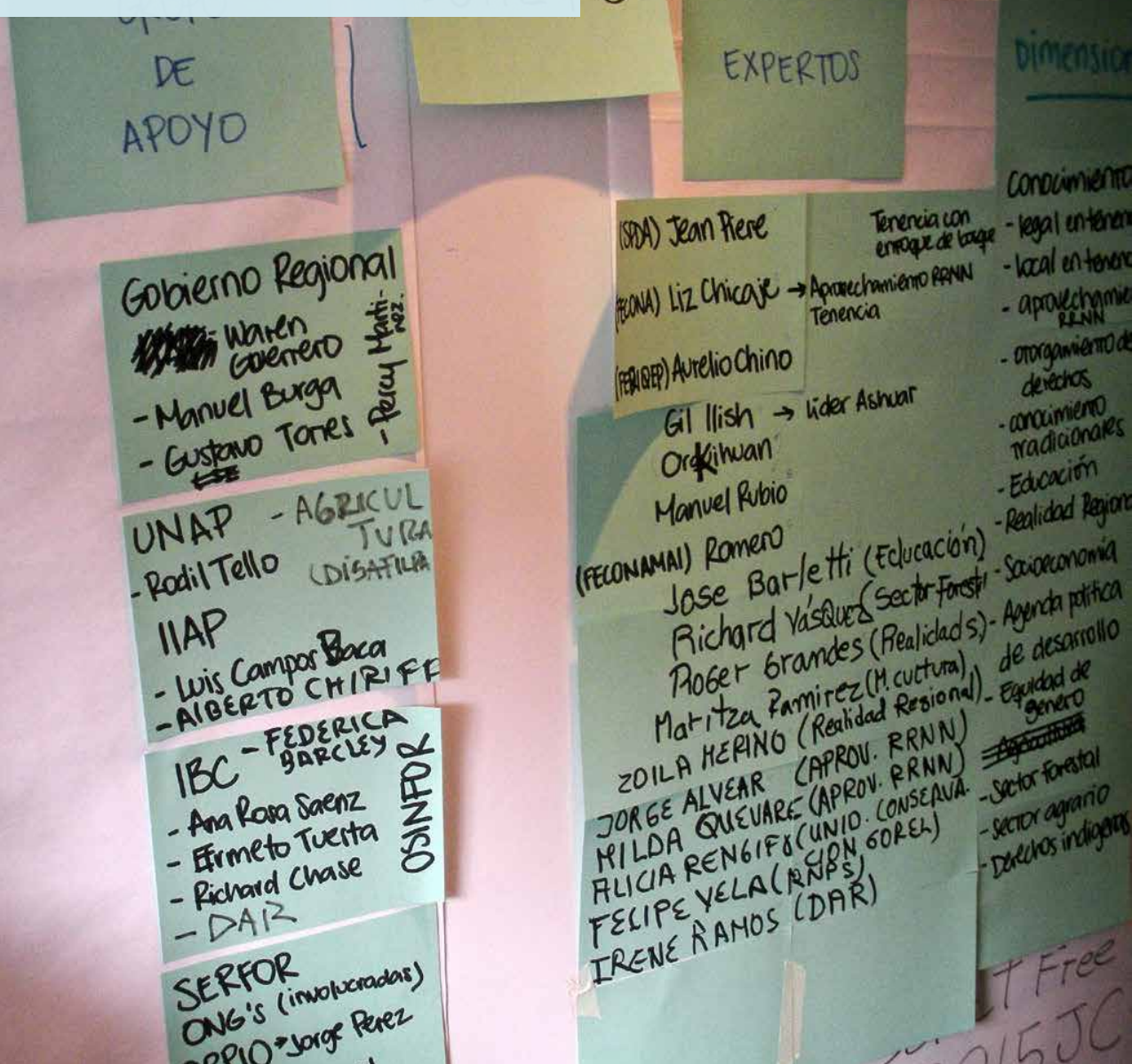


La première tâche du groupe d'experts est de définir le système et de parvenir à s'accorder sur sa définition. Il faut que les membres soient d'accord sur ce qu'ils considéreront collectivement comme le système de sécurité du foncier forestier dont ils vont explorer l'avenir. Ils devront aussi au préalable tous s'accorder sur une règle collective pour la prise de décisions. Ils suivront cette règle pour parvenir à des accords et pour avancer en cas d'opinions divergentes entre les participants. Les membres sont libres de choisir la règle qu'ils souhaitent, mais doivent tous être d'accord sans exception pour respecter cette règle par la suite.

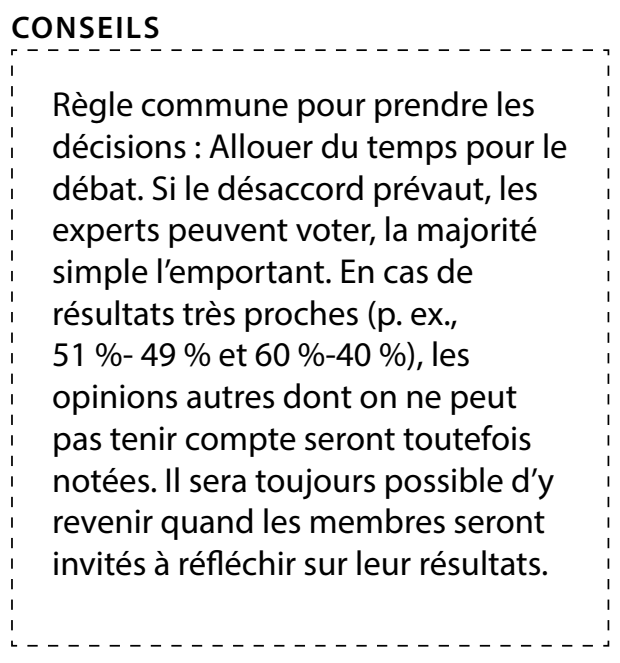

\section{2 Étape 2 : Identifier et définir les facteurs de changement}

Une fois que le système est clairement posé, le premier point d'étape est une liste de facteurs de changement bien définis. Un facteur de changement peut transformer le système de manière importante à l'avenir, quelle que soit la direction prise.

\subsubsection{Comprendre le système, voir loin}

Quand les membres du groupe d'experts identifient des facteurs de changement, ils peuvent avoir des difficultés à se projeter dans l'avenir et à l'horizon temporel qu'ils ont décidé. De même, il n'est pas toujours facile pour les personnes d'avoir une représentation mentale claire du système. Pour résoudre ces difficultés, un exercice pratique est particulièrement utile.

Il s'agit, en deux tours, de demander à chaque membre d'indiquer sa plus grande crainte et son plus grand espoir pour l'avenir de la sécurité du foncier forestier à (nom du lieu) d'ici (échéance convenue).

Chaque participant écrit une courte phrase ou quelques mots sur des fiches de couleur (des couleurs différentes peuvent être utilisées pour les espoirs et les craintes). Quand les fiches sont remplies, elles sont affichées sur le support et les participants précisent en quelques mots leurs idées. L'animateur prend les fiches pour les rassembler sous les « craintes » ou sous les « espoirs". 
Toutes les informations sont notées et restent affichées pour que les participants puissent les consulter pendant les sessions de travail. On leur demandera immédiatement après de déterminer les facteurs de changement (qui sont susceptibles de transformer significativement le système à l'avenir). La réponse à cette question n'est pas toujours simple. Cependant, quand les membres ont identifié leurs craintes et leurs espoirs, il est plus facile de travailler sur ces facteurs (parce qu'ils sont, pour certains, la cause des craintes et des espoirs indiqués).

\subsubsection{Dresser la liste des facteurs de changement}

Les facteurs de changement à identifier sont ceux qui ont pesé par le passé, influent sur le présent et influeront à l'avenir sur l'évolution du territoire. Voici le processus à suivre pour établir la liste des facteurs de changement potentiels :

- Utiliser des fiches de couleur pour que les personnes s'expriment librement et que la contribution des membres du groupe soit équilibrée.

- Inviter les participants à écrire séparément les facteurs de changement qu'ils considèrent importants, un par fiche.

- Ramasser, lire et afficher toutes les fiches sur un support visible de tous sans qu'il y ait besoin de les relire.

\section{CONSEILS}

Mettre à profit ce court échange à bâtons rompus comme moyen de briser la glace pour que les participants fassent connaissance. Dans ce but, ceux-ci se présentent en indiquant les connaissances dont ils disposent, l'expérience et l'expertise liées à l'avenir de la sécurité du foncier forestier.

Ensuite, ils expliquent brièvement leurs espoirs et leurs craintes. Trois minutes par personnes sont suffisantes pour se présenter et expliquer ses espoirs et ses craintes. L'un des animateurs fera le gardien du temps pour s'assurer que les personnes ne dépassent pas le temps imparti.

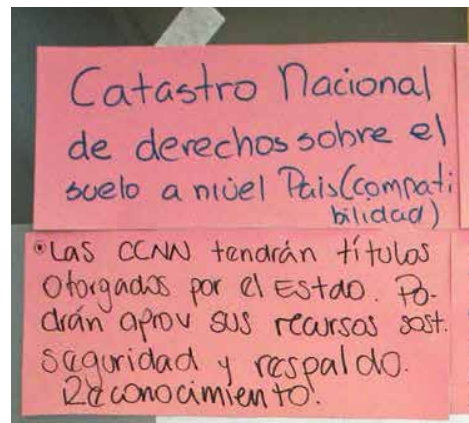

DERECMOR ESTATUTARIOI Y CONSUETUDINARROT BIEN IDENTI FICDDOY $y$ BIEN AURADOT CORO DASE DEL DESARRULCO INTEGRA Que la tierra sea de quien hace uso de ella sosteniblemente

Tenencia ordenada y legal del bosque respetando los de rechos de las poblaciones en base a una legis ación a decua a una legislacion da Existe un adecuado OT. Los trámites soll rápidos, estable Adecuada revisión del expe diente técnico.

- Concimiento de derechos de cell $\rightarrow$ titulos - Manejo local sostanible - Planes de mancio locales - Mejor distribución de bencfio
Que existan leyes bient? establecidas sobre tenenci manejo de recursos pores ann y essas se cumplan.

UN SISTEMA IE TENENCI FORESTAL SIN RIEGSD DE COMERCIO JE TEERRAS QUE AFECTE SUS CAPACIDADES ECOSISTÉMICAS, Y CON RES A COS DERECHOS DE COM. NATTI

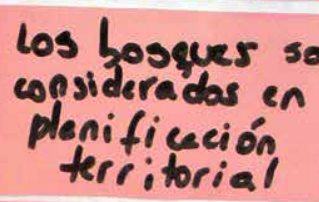

Tener un proceso de veforma de tenencia clara y conderi gavantizando sequridad à los pstreadies

Affichage des espoirs au Pérou 
- Règle 2 : Une phrase n'est pas un facteur de changement; il faut reformuler.

- Règle 3 : Un énoncé négatif n'est pas un facteur de changement ; il faut reformuler.

- Règle 4 : La référence à un élément matériel n’est pas un facteur de changement ; il faut reformuler.

- Règle 5 : Les facteurs de changement évoqués pour lesquels il serait impossible d'identifier des configurations futures différentes ne peuvent être conservés. La question très utile est la suivante : "Est-ce un facteur de changement ou une configuration future ? " Une configuration décrit la situation éventuelle d'un facteur à l'avenir, avec ses caractéristiques.

Les fiches qui respectent ces cinq règles ne font pas l'objet d'un débat. Toutes les autres doivent être discutées. La figure 5 présente des exemples de reformulation des facteurs de changement.

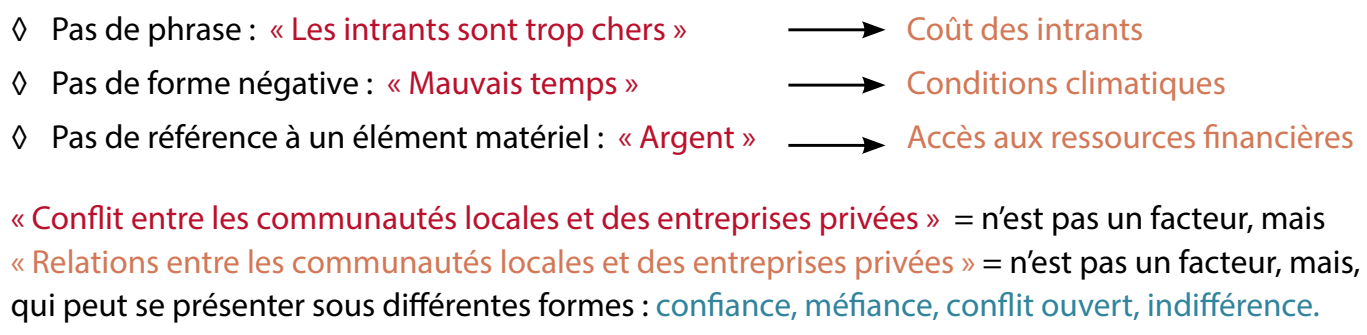

Figure 5. Exemples de reformulation des facteurs de changement.

\subsubsection{Reformuler l'énoncé des facteurs de changement}

La façon dont un facteur de changement est formulé permet souvent de mieux comprendre la réalité, ainsi que les points de vue et les attentes de chaque participant. Les participants tendent à expliquer la réalité en écrivant par exemple : "Le gouvernement n'a pas la volonté de s'occuper de nous ", "Les lois ne correspondent pas à la réalité ", "Éducation interculturelle ". L'animateur doit noter ces points de vue parce qu'ils sont utiles pour les étapes ultérieures. La liste finale des facteurs de changement est ensuite établie. Il convient ensuite de la réviser pour distinguer les facteurs internes des facteurs externes. Voici la définition de ces deux types de facteurs :

- Les facteurs externes ne peuvent pas être contrôlés par les acteurs directement impliqués dans le système. Ils sont propres au contexte ou à l'environnement dans lequel s'inscrit le système.

- Les facteurs internes peuvent être contrôlés par les acteurs du système. Certains acteurs ou l'ensemble de ceux-ci ont le pouvoir de modifier la configuration de ces facteurs à l'avenir.

On décide qu'un facteur est interne ou externe en fonction de la façon dont le système a été défini et, en particulier, de son périmètre géographique et de ses acteurs. Un même facteur de changement peut être considéré interne au niveau national 
(p. ex., " orientations politiques nationales relatives à la gestion forestière »), mais externe au niveau d'un bassin versant (car les acteurs locaux n'ont aucune possibilité de contrôle ou d'influence). Les tableaux 2 et 3 exposent des exemples de facteurs internes et externes observés dans les ateliers de formation.

Établir deux listes finales : l'une pour les facteurs internes et l'autre pour les facteurs externes. Les tableaux 2 et 3 exposent des exemples de facteurs internes et externes observés lors de l'atelier de formation de Bogor.

Tableau 2. Facteurs internes évoqués lors de la formation des animateurs à Bogor (Indonésie).

\begin{tabular}{|c|c|}
\hline Nom du facteur & Définition \\
\hline $\begin{array}{l}\text { Mise en œuvre des } \\
\text { politiques publiques }\end{array}$ & $\begin{array}{l}\text { Mise en œuvre par le gouvernement local des politiques du } \\
\text { gouvernement central relatives à l'utilisation des espaces } \\
\text { boisés }\end{array}$ \\
\hline Gouvernance locale & $\begin{array}{l}\text { Gouvernance politique, économique et administrative } \\
\text { assurée par le gouvernement local dans le domaine du } \\
\text { foncier forestier }\end{array}$ \\
\hline Processus d'autorisation & $\begin{array}{l}\text { Processus relatif à l'obtention d'un permis d'exploitation des } \\
\text { forêts par le gouvernement, y compris les procédures de } \\
\text { gouvernance et la communication des informations }\end{array}$ \\
\hline Liens entre les secteurs & $\begin{array}{l}\text { Relations entre les secteurs du gouvernement local lors de la } \\
\text { détermination du mode de gestion durable des forêts }\end{array}$ \\
\hline Répartition du pouvoir & $\begin{array}{l}\text { Répartition du pouvoir entre les parties prenantes lors de } \\
\text { l'obtention des avantages tirés des forêts }\end{array}$ \\
\hline $\begin{array}{l}\text { Connaissance du } \\
\text { gouvernement local }\end{array}$ & $\begin{array}{l}\text { Connaissance de l'appareil régional sur la politique publique } \\
\text { d'accès au domaine forestier }\end{array}$ \\
\hline Aide aux organisations & $\begin{array}{l}\text { Capacité du gouvernement/des parties prenantes à } \\
\text { accompagner les institutions des communautés forestières }\end{array}$ \\
\hline $\begin{array}{l}\text { Dépendance vis-à-vis de } \\
\text { la forêt }\end{array}$ & $\begin{array}{l}\text { Dépendance de la population vis-à-vis de la forêt (comme } \\
\text { principale source de moyens de subsistance) }\end{array}$ \\
\hline Accès aux capitaux & $\begin{array}{l}\text { Accès aux capitaux pour les groupes qui disposent d'un droit } \\
\text { légal d'usage sur la forêt (facilité d'accès et confiance) }\end{array}$ \\
\hline $\begin{array}{l}\text { Investissements du } \\
\text { secteur privé }\end{array}$ & $\begin{array}{l}\text { Investissements de capitaux par le secteur économique dans } \\
\text { l'exploitation de produits forestiers }\end{array}$ \\
\hline Budget du gouvernement & $\begin{array}{l}\text { Ressources financières allouées par le gouvernement local à } \\
\text { la réforme du foncier forestier }\end{array}$ \\
\hline Croissance économique & Croissance économique liée au besoin en terres forestières \\
\hline $\begin{array}{l}\text { Croissance } \\
\text { démographique }\end{array}$ & $\begin{array}{l}\text { Croissance démographique liée au besoin en terres } \\
\text { forestières }\end{array}$ \\
\hline
\end{tabular}


Tableau 2. Suite

\begin{tabular}{|c|c|}
\hline Nom du facteur & Définition \\
\hline $\begin{array}{l}\text { Participation de la } \\
\text { communauté }\end{array}$ & $\begin{array}{l}\text { Community participation in the implementation of forest } \\
\text { tenure reform }\end{array}$ \\
\hline Capacité de gestion & $\begin{array}{l}\text { Capacité de la communauté à développer l'exploitation de } \\
\text { produits forestiers }\end{array}$ \\
\hline « Hak Ulayat » & $\begin{array}{l}\text { Droits sur les terres forestières appartenant depuis des } \\
\text { générations aux populations autochtones }\end{array}$ \\
\hline $\begin{array}{l}\text { Institutions } \\
\text { communautaires }\end{array}$ & $\begin{array}{l}\text { Capacité des institutions communautaires à mettre en } \\
\text { œuvre la politique de gestion des terres et l'exploitation des } \\
\text { produits forestiers }\end{array}$ \\
\hline $\begin{array}{l}\text { Droits/accès à la terre et à } \\
\text { la forêt }\end{array}$ & $\begin{array}{l}\text { Droit d'usage et accès des parties prenantes à la terre et à la } \\
\text { forêt }\end{array}$ \\
\hline Capacité d'organisation & Capacité des communautés à s'organiser \\
\hline Attitude du secteur privé & $\begin{array}{l}\text { Attitude du secteur privé envers la réglementation et la } \\
\text { politique concernant le régime foncier forestier }\end{array}$ \\
\hline $\begin{array}{l}\text { Sensibilisation de la } \\
\text { population }\end{array}$ & $\begin{array}{l}\text { Connaissance par la population de ses droits et obligations } \\
\text { vis-à-vis de l'usage des forêts }\end{array}$ \\
\hline $\begin{array}{l}\text { Définition des limites } \\
\text { traditionnelles }\end{array}$ & $\begin{array}{l}\text { Definition by indigenous peoples of boundaries of their } \\
\text { territories based on natural boundaries recognized by them } \\
\text { for generations }\end{array}$ \\
\hline Conversion des terres & $\begin{array}{l}\text { Conversion des terres forestières, qui étaient auparavant } \\
\text { des forêts appartenant à l'État et qui ne le sont plus, liée au } \\
\text { processus de détermination de statut par KLHKa }\end{array}$ \\
\hline $\begin{array}{l}\text { Information et } \\
\text { communication }\end{array}$ & $\begin{array}{l}\text { Système d'information et de communication grâce au } \\
\text { référencement géospatial (cartographie des zones boisées) }\end{array}$ \\
\hline
\end{tabular}

a Kementerian Lingkungan Hidup dan Kehutanan (KLHK) / Ministère de l'Environnement et des Forêts (MoEF)

Tableau 3. Facteurs externes évoqués lors de la formation des animateurs à Bogor (Indonésie).

\begin{tabular}{ll}
\hline Nom du facteur & Définition \\
\hline $\begin{array}{l}\text { Politiques } \\
\text { nationales }\end{array}$ & $\begin{array}{l}\text { Politiques du gouvernement central concernant les droits de la } \\
\text { population relatifs à l'accès et à l'usage de la forêt }\end{array}$ \\
Application de la loi & $\begin{array}{l}\text { Application de la loi concernant le statut des terres du domaine } \\
\text { public précédemment certifiées avant la mise en place de la } \\
\text { nouvelle réforme foncière }\end{array}$ \\
Autorisation & $\begin{array}{l}\text { Processus par lequel le gouvernement central (ministère LHK) } \\
\text { octroie à la population des droits d'accès et de gestion de la } \\
\text { forêt dans le cadre de zones spécifiques (HKm, HD, HTR et autres } \\
\text { programmes CBFM) }\end{array}$ \\
\hline
\end{tabular}


Tableau 3. Suite

\begin{tabular}{|c|c|}
\hline Nom du facteur & Définition \\
\hline $\begin{array}{l}\text { Gouvernance } \\
\text { centrale }\end{array}$ & $\begin{array}{l}\text { Gouvernance politique, économique et administrative assurée par le } \\
\text { gouvernement central concernant le régime foncier forestier }\end{array}$ \\
\hline $\begin{array}{l}\text { Réglementation des } \\
\text { forêts d'État }\end{array}$ & $\begin{array}{l}\text { Dispositions prises par le ministère LHK pour définir le statut et } \\
\text { réglementer le fonctionnement des forêts d'État }\end{array}$ \\
\hline Budget national & $\begin{array}{l}\text { Budget alloué par le gouvernement pour la réforme du foncier } \\
\text { forestier }\end{array}$ \\
\hline $\begin{array}{l}\text { Conditions } \\
\text { géographiques }\end{array}$ & $\begin{array}{l}\text { Paramètres géographiques d'une région constituée d'îles affectant } \\
\text { la sécurité du foncier forestier (à comparer à la situation d'une } \\
\text { zone continentale); cela concerne les éléments biophysiques et } \\
\text { socioculturels comme l'accessibilité }\end{array}$ \\
\hline
\end{tabular}

\subsubsection{Définir les facteurs de changement}

Pour chaque facteur de changement identifié, les participants doivent s'accorder sur une définition commune et claire. Cette définition est en général une phrase expliquant la nature du facteur. Cette étape est extrêmement importante, car elle conduit à un point d'étape. Les définitions seront systématiquement utilisées à l'étape suivante lorsque l'on procédera à l'analyse structurelle. La qualité de cette analyse dépend de la qualité et de la précision de la définition. La qualité de cette définition sera aussi déterminante lors de l'étape après la suivante pour identifier la future configuration des facteurs de changement. Cette définition doit permettre de cerner les configurations plausibles de ce facteur à l'avenir.

Noter toutes les définitions afin de pouvoir les utiliser lors des étapes suivantes. Fournir à tous les participants un tableau exhaustif des facteurs internes et externes dont ils ont convenu, avec leur définition complète.
- Choisir entre 20 et 60 facteurs de changement internes (le logiciel peut en gérer 73).

- Prendre le temps de réfléchir collectivement au résultat obtenu et s'assurer qu'on n'a oublié aucun facteur important.

- S'assurer qu'aucun élément important ne manque (social, technique, économique,

- Veiller à ce que cette définition soit suffisamment détaillée (voir les exemples de définition dans le tableau 2). environnemental, politique, etc.). 


\section{Encadré 10. Les facteurs de changement dans le cas du Pérou}

Les participants de Madre de Dios ont identifié 27 facteurs, et ceux de Loreto 42.

\section{Enseignements :}

- Définir les facteurs lors du débat sur les fiches complétées par les participants. Quand les fiches sont regroupées et que les participants décident si certaines se ressemblent ou non, cela suscite souvent un débat. Un membre du groupe de travail doit prendre des notes. Elles seront essentielles lors de la délibération qui s'ensuivra sur la définition des facteurs.

- Faire préciser leurs idées aux participants en leur demandant à chaque fois que c'est nécessaire : "Que signifie ce facteur pour vous ? „C'est important pour mettre en évidence les problèmes qu'ils veulent signaler. Le temps est ainsi mieux employé. Au Pérou, à la fin du premier jour de l'atelier, le groupe de travail devait encore affiner ces définitions.

- Donner aux participants la liste des facteurs avec leur définition et les inviter à commenter.

\section{3 Étape 3 : Identifier et choisir les facteurs moteurs}

Quand les facteurs internes ont été identifiés et clairement définis, analyser systématiquement les relations qui existent entre eux. Ceci implique d'évaluer I'influence mutuelle directe des facteurs internes. L'existence ou l'absence d'influence directe entre les facteurs entraîne une classification de ceux-ci, qui contribue à structurer le système. Pour cette raison, ce travail s'appelle aussi « analyse structurelle ». Les résultats sont entrés dans une matrice liée à des graphiques et des tableaux qui fournit immédiatement les informations nécessaires pour choisir les facteurs moteurs. Les facteurs moteurs sont les plus influents et puissants du système.

\subsubsection{Mesurer les influences directes}

La compréhension des relations entre les facteurs est capitale pour l'élaboration de scénarios multidimensionnels du futur de la sécurité du foncier forestier. Cela nous permet de comprendre les forces dynamiques du système, la façon dont il évolue, et aussi de comprendre la nature des interactions en jeu et les relations entre les composantes essentielles de la sécurité du foncier forestier d'un point de vue dynamique. Les membres du groupe d'experts débattent des influences directes au cours d'une réunion dédiée à cette question dans le cadre de l'analyse structurelle. 
L'évaluation des influences directes entre les facteurs est basée sur un code de notation convenu. Un système binaire représente l'influence directe entre deux facteurs $\{0 ; 1\}$. En l'absence d'influence directe d'un facteur $A$ sur un facteur $B$, la relation $A B$ est égale à zéro $(\mathrm{AB}=0)$; avec un lien direct d'influence de $\mathrm{A}$ vers $\mathrm{B}$, la relation est de 1 $(\mathrm{AB}=1)$. La relation entre $\mathrm{B}$ et $\mathrm{A}$ est aussi évaluée et peut être égale à 0 ou $1(\mathrm{BA}=0$ ou $\mathrm{BA}=1)$.

Il y a une influence directe du facteur A sur le facteur B si tout changement touchant le facteur A provoque un changement du facteur $B$, et que ce changement peut être clairement et logiquement expliqué. Pour s'assurer que l'influence repérée entre le facteur A et le facteur B est directe, une explication est nécessaire. Cette explication élimine les trois principales erreurs énumérées ci-après.

- Confusion dans la direction du lien de causalité* : Une personne pense que $\mathrm{A}$ influence $\mathrm{B}$, alors que c'est le contraire. Cela arrive fréquemment au début de l'analyse d'influence/ dépendance $(\mathrm{I} / \mathrm{D})$.

- Cependant, en général, les participants eux-mêmes le corrigent rapidement. Il est aussi possible que deux facteurs s'influencent directement réciproquement.

- Influence indirecte : $A$ influence $B$ et $B$ influence $C$, par conséquent $\mathrm{A}$ influence $\mathrm{C}: \mathrm{Il}$ s'agit d'une influence indirecte qui ne doit pas être prise en compte pour la raison indiquée plus haut. Lorsqu'il y a une relation causale indirecte entre deux facteurs, il est généralement possible d'identifier le facteur intermédiaire qui les relie. Si ce facteur

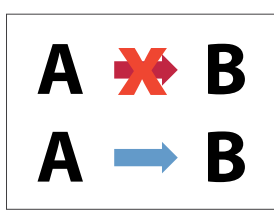
fait partie du système (déjà dans la liste), il s'agit d'un cas de transitivité. Cependant, si le facteur n'est pas pris en compte, la question de sa prise en compte doit être débattue. Si les experts sont d'accord pour l'inclure, la liste est modifiée en conséquence et l'analyse I/D est entreprise avec ce facteur supplémentaire.

- Covariation : quelqu'un pense que deux facteurs sont liés parce qu'ils évoluent de façon similaire, mais un autre facteur influence ces deux facteurs simultanément : $\mathrm{C}$ influence à la fois $\mathrm{A}$ et $\mathrm{B}$, mais $\mathrm{A}$ et $\mathrm{B}$ ne sont pas directement liés.

Quand l'influence directe d'un facteur sur tous les autres a été

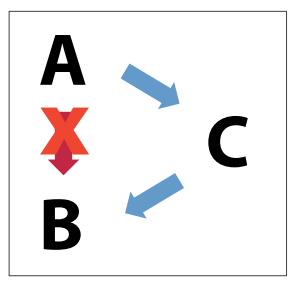
débattue et que les parties sont d'accord, le groupe de travail prépare immédiatement les résultats dans la matrice d'influence/dépendance (matrice I/D). Cette matrice est un tableau de $\mathrm{n}$ lignes et $\mathrm{n}$ colonnes, dans lequel $\mathrm{n}$ est le nombre total de facteurs (voir la section 3.3.1). 
Chaque facteur fait l'objet d'un débat jusqu'à ce que la matrice soit complétée avec les valeurs convenues.

Ce processus est assez long au début, mais avec la pratique, les experts y passent moins de temps. Les principales tâches de l'animateur sont les suivantes :

- Aider le groupe d'experts à ne considérer que les relations directes causales. L'animateur doit toujours être concentré sur les trois sources d'erreur évoquées ci-dessus. En cas de doute, l'animateur doit questionner le groupe sur l'existence d'une influence directe ou indirecte, ou d'une influence inverse ou d'une coévolution. Il ne s'agit pas d'insister pour que le groupe accepte l'opinion de l'animateur, mais de veiller à ce que ce groupe décide en fonction d'arguments logiques et acceptables.

- Veiller à chaque fois que la délibération sur les influences directes s'appuie systématiquement sur la définition du facteur et pas simplement sur son nom court. En se basant sur la définition, il est plus facile de répondre à la question qui est plus précise. En se reportant systématiquement à la définition tout au long de l'analyse structurelle, les réponses seront plus précises. Cela évite la confusion et les malentendus à cause des divergences d'interprétation pouvant survenir si l'on se base uniquement sur le nom des facteurs (voir l'exemple ci-dessous).

\section{Encadré 11. Se baser sur la définition et pas sur le nom de la variable : exemple}

Prenons un exemple dans le tableau 2 avec deux facteurs (" gouvernance locale » et " Attitude du secteur privé ») évoqués lors de la formation sur la sécurité du foncier forestier en Indonésie.

Incorrect : La "gouvernance locale » a-t-elle une influence directe sur "l'attitude du secteur privé»?

Correct : La gouvernance politique, économique et administrative par le gouvernement local dans le domaine du régime foncier forestier a-t-elle une influence directe sur I'« attitude du secteur privé envers la réglementation et la politique concernant le foncier forestier »?

En d'autres termes, cela revient à poser la question suivante : Si la gouvernance politique, économique et administrative par le gouvernement local dans le domaine du régime foncier forestier devait changer de manière significative, cela transformerait-il directement l'attitude du secteur privé envers la réglementation et la politique concernant le régime foncier forestier? 


\subsubsection{Mise en œuvre}

Le temps nécessaire pour achever le processus dépend du nombre de facteurs de changement. Les participants ont généralement besoin de temps pour manier le concept d'influence directe et l'appliquer. Comme il s'agit d'un processus d'apprentissage par la pratique, l'analyse avance progressivement plus vite. L'expérience montre que, après une demi-journée d'apprentissage où l'on peut débattre au mieux de deux ou trois facteurs, la « vitesse de croisière " de l'analyse structurelle est d'environ 30 minutes par facteur pour un système qui en comprend 50 (l'analyse de certains facteurs demande cependant plus de 30 minutes; voir l'encadré 12). Cela signifie qu'il faut prévoir une session de trois jours pleins. Lorsque c'est absolument nécessaire, le groupe d'experts peut être scindé en deux sous-groupes qui traiteront chacun de la moitié des facteurs. Cependant, cela ne peut être qu'un «Plan B » quand il n'y a aucune possibilité d'obtenir des résultats dans le temps imparti. La durée de la réunion sur l'analyse structurelle doit être prévue à l'avance pour s'assurer que la totalité du groupe peut y prendre part. Le débat sur les influences directes nécessite d'utiliser des supports comme celui présenté ci-dessous.

Utiliser le support ainsi :

- Préparation : Donner un nom court à tous les facteurs choisis (un "diminutif »). Écrire ces noms sur des fiches. Placer ces fiches avec le nom des facteurs (p. ex., Facteur A) sur le support.

- Étape 1 : Discuter ensemble de chaque facteur un par un. Poser la question suivante à l'ensemble des participants : "Le facteur A influence-t-il directement le facteur B ? » Si la réponse est non, le facteur B est placé dans la colonne « 0 ». Si la réponse est oui, le facteur $B$ est placé dans la colonne " 1 ».

- Étape 2 : Inscrire les résultats dans la matrice. Voir la figure 6 qui présente un exemple de cas rencontré lors de l'analyse I/D.

- Étape 3 : Placer en haut la fiche avec le nom du facteur suivant (p. ex., facteur B). Les participants répètent le processus jusqu’à ce que tous les facteurs aient été analysés.

\section{Encadré 12. Organiser l'analyse des}

L'analyse des influences mutuelles ne prend pas le même temps selon le type de facteur. Au Pérou, certains facteurs d'ordre juridique ont exigé plus de débat que d'autres. Au Pérou et en Indonésie, il a été nécessaire de scinder le groupe d'experts en deux ou trois sousgroupes. Au Pérou, le groupe d'experts scindé en deux a terminé l'analyse I/D de 42 facteurs en une journée (au moins huit heures de véritable travail). En Indonésie, les groupes supplémentaire s'est avérée nécessaire pour vérifier les résultats des groupes. La principale raison pour répartir le travail entre des sousgroupes est que certains facteurs demandent plus de 30 minutes de débat. Étant donné la durée de l'atelier, le groupe a été divisé afin de terminer à I'heure. 


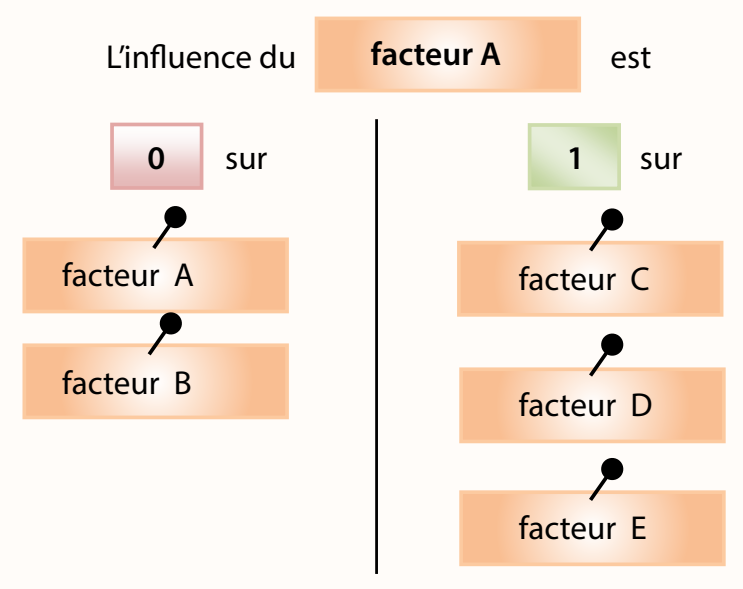

Figure 6. Exemple de cas rencontré lors de l'analyse I/D.

En cas de désaccord, appliquer la règle de prise de décisions adoptée au début de la démarche prospective.

Certaines techniques d'animation peuvent accélérer la délibération, notamment cellesci pour favoriser le débat et aider les participants à décider :

- Préparation : Préparer trois grands supports verticaux sur lesquels on pourra afficher toutes les fiches. En haut des supports, écrire « 0 » sur le premier, «Espace de débat » sur le deuxième et « 1 » sur le troisième.

- Étape 1 : Choisir le facteur dont vous souhaitez débattre (p. ex., facteur 1) et le placer en haut de l'« Espace de débat ». Mettre toutes les fiches restantes dans l'espace « $0 »$.

- Étape 2 : Lors d'un premier tour, inviter tous les participants à choisir les fiches correspondant aux facteurs qui, de leur avis, sont directement influencés par le facteur 1 et ensuite les déplacer dans l'espace " 1 ». C'est une décision individuelle. Cependant, la personne doit justifier sa décision de déplacer la fiche de « 0 » à " 1 ». Cette opération ne doit pas durer plus de trois minutes.

- Étape 3 : Lors d'un second tour, inviter les participants à revoir individuellement les propositions faites lors du premier tour. S'ils ont des doutes ou ne sont pas d'accord avec les déplacements proposés, ils placent les fiches en question dans l'«Espace de débat ». Prévoir trois minutes pour cela. Discuter des fiches placées dans l'« Espace de débat " pour décider collectivement s'il faut les mettre en « 1 » 
ou en « 0 » puis les déplacer en conséquence. Prévoir 10 minutes pour cette opération, pas plus.

- Étape 4 : Inviter les participants à revoir l'espace « 0 » pour s'assurer que, à la lumière de leur délibération, il n'y a pas d'autre fiche à déplacer (bien entendu, il n'y a pas lieu de rediscuter des fiches qui viennent d'être déplacées de l' "Espace de débat » vers « $0 »)$. Débattre collectivement de tout nouveau déplacement de fiche et décider de l'endroit où elle doit aller. Prévoir 5 minutes pour cela, pas plus.

- Étape 5 : Inviter tous les participants à revoir le tout et à s'accorder sur le résultat. Inscrire les résultats dans la matrice. Rassembler toutes les fiches dans un espace (c'est plus rapide de choisir celui où il y en a le plus) et nommer cet espace " 0 » avant de recommencer le processus.
CONSEILS

- Les participants apprécient beaucoup le processus interactif de l'« Espace de débat ». Ils se sentent plus directement impliqués dans la décision et cela fait gagner beaucoup de temps. Cependant, cet espace ne peut pas être utilisé dès le départ. Les participants doivent avoir acquis les bases et pratiquer un peu avant d'être capables de décider des influences directes individuellement et ensuite collectivement.

- Utiliser ce processus de manière encore plus interactive en offrant aux participants d'animer le débat de groupe. Les animateurs « officiels » veillent à ce que l'atelier suive les principes convenus.

- En cas de désaccord et s'il y a peu d'écart lors du vote sur les influences directes, changer la valeur entrée dans la matrice (si on le souhaite) pour réaliser un test de sensibilité. À cet effet, mettre en surbrillance la cellule correspondante en jaune. Cela permet de raccourcir le débat, mais il ne faut pas y recourir systématiquement. Une trop grande variation pourrait affecter la sensibilité des résultats.

Parfois, lors de la mesure de l'influence, on remarque que certains facteurs manquent. Par exemple, nous avons un facteur A qui influence indirectement les facteurs B, C, D et $\mathrm{E}$. L'influence est indirecte, car la séquence correcte est $\mathrm{A}$ influence $\mathrm{X}$ et $\mathrm{X}$ influence B, C,et E. Mais nous ne connaissons pas le facteur X. Ainsi, le facteur A, qui devrait être un facteur moteur devient un facteur d'effet. Exemple observé au Pérou :

"Qualité de l'éducation » : ce facteur a été considéré très important, mais le raisonnement était le suivant : "Si nous améliorons la qualité de l'éducation, la sécurité foncière se verra dans 10 ans (quand les enfants éduqués seront adultes). On a donc considéré qu'il influençait indirectement de nombreux facteurs. Par conséquent, dans le graphique des influences directes, il apparaissait comme " facteur d'effet ». Enfin, malgré les résultats, les participants ont convenu de le mettre dans la catégorie des facteurs moteurs. 
Enseignements : Il doit y avoir consensus sur la signification de "direct ». Direct signifie que si je change A, B change aussi relativement rapidement. Il peut y avoir plus de facteurs intermédiaires comme le facteur $\mathrm{X}$ mentionné auparavant, si les experts ont défini les facteurs plus spécifiquement.

\subsubsection{Enregistrer les données et procéder au calcul automatique}

La matrice I/D peut être un grand tableau. Si 50 facteurs ont été identifiés, la matrice I/D est un tableau carré de 2500 cellules! Chaque cellule sera remplie avec les résultats de l'analyse structurelle, sauf les cellules de la diagonale correspondant à l'influence d'un facteur sur lui-même (par définition, cela ne peut pas être compté comme influence directe).

L'analyse du contenu de la matrice repose par conséquent sur un calcul automatisé. À cette fin, un simple programme informatique permet de visualiser les résultats. La manipulation du logiciel exige un minimum d'expertise, car presque tous les calculs et affichages sont déjà dans la feuille de calcul grâce aux liens automatiques entre différentes feuilles de calcul. En fait, seules deux opérations sont nécessaires : i) entrer le nom des facteurs une fois et ii) remplir la matrice des influences directes une fois. La structure et les fonctions des feuilles de calcul sont décrites ci-après.

\section{La feuille de travail « Variables' influence »}

La matrice d'influence directe se trouve sur la feuille de travail intitulée "Variables' influence ». Elle doit être remplie avec les résultats du travail réalisé avec les experts au cours de l'atelier/des ateliers. Chaque facteur est d'abord enregistré par son nom court dans la première colonne de cette matrice (colonne B). C'est à faire seulement une fois pour chaque facteur.

Le nom de chaque facteur apparaîtra automatiquement sur la première ligne de titre horizontal de la matrice (voir la figure 7 ci-dessous basée sur un exercice de prospective appliqué à l'avenir des zones rurales en Afrique). La valeur correspondant à l'influence de chaque facteur sur les autres est inscrite dans la matrice, comme convenu par les experts au cours de l'atelier. La matrice est remplie ligne par ligne, et pour chaque ligne, de la gauche vers la droite. Une chaîne de liens automatiques entre les différentes matrices, tableaux et graphiques permet d'afficher immédiatement tous les résultats (voir la figure 8 ci-dessous).

La feuille de travail "Variables' influence » affiche aussi quatre tableaux supplémentaires qui mesurent le poids de chaque facteur dans le système. Ces tableaux se trouvent en bas de la matrice :

- Un tableau des influences directes globales

- Un tableau des dépendances directes globales

- Un tableau des forces directes globales

- Un tableau des forces directes pondérées 


\section{La feuille de travail « Variable's total influence »}

Cette feuille de travail présente d'abord une matrice des influences indirectes entre les facteurs. Cette matrice est directement liée à la matrice de la première feuille de travail. Il n'est pas besoin de la remplir. Quand la matrice des influences directes est complètement remplie, celles des influences indirectes est aussi complètement remplie sans intervention supplémentaire.

Dans cette matrice des influences indirectes, on trouve quatre tableaux semblables à ceux de la première feuille de travail. Ils calculent automatiquement les valeurs suivantes :

- Influence indirecte globale

- Dépendance indirecte globale

- Force indirecte globale

- Force pondérée indirecte globale

Sous ces quatre tableaux, il y a une matrice des infuences totales de chaque facteur, dans laquelle viennent s'ajouter automatiquement les influences directes et indirectes. De même, dans cette matrice, on peut trouver les quatre tableaux suivants :

- Influence totale globale

- Dépendance totale globale

- Force totale globale

- Force totale globale pondérée

Les participants de l'atelier de Lampung en Indonésie ont été répartis en trois groupes qui ont dirigé la session chacun leur tour.

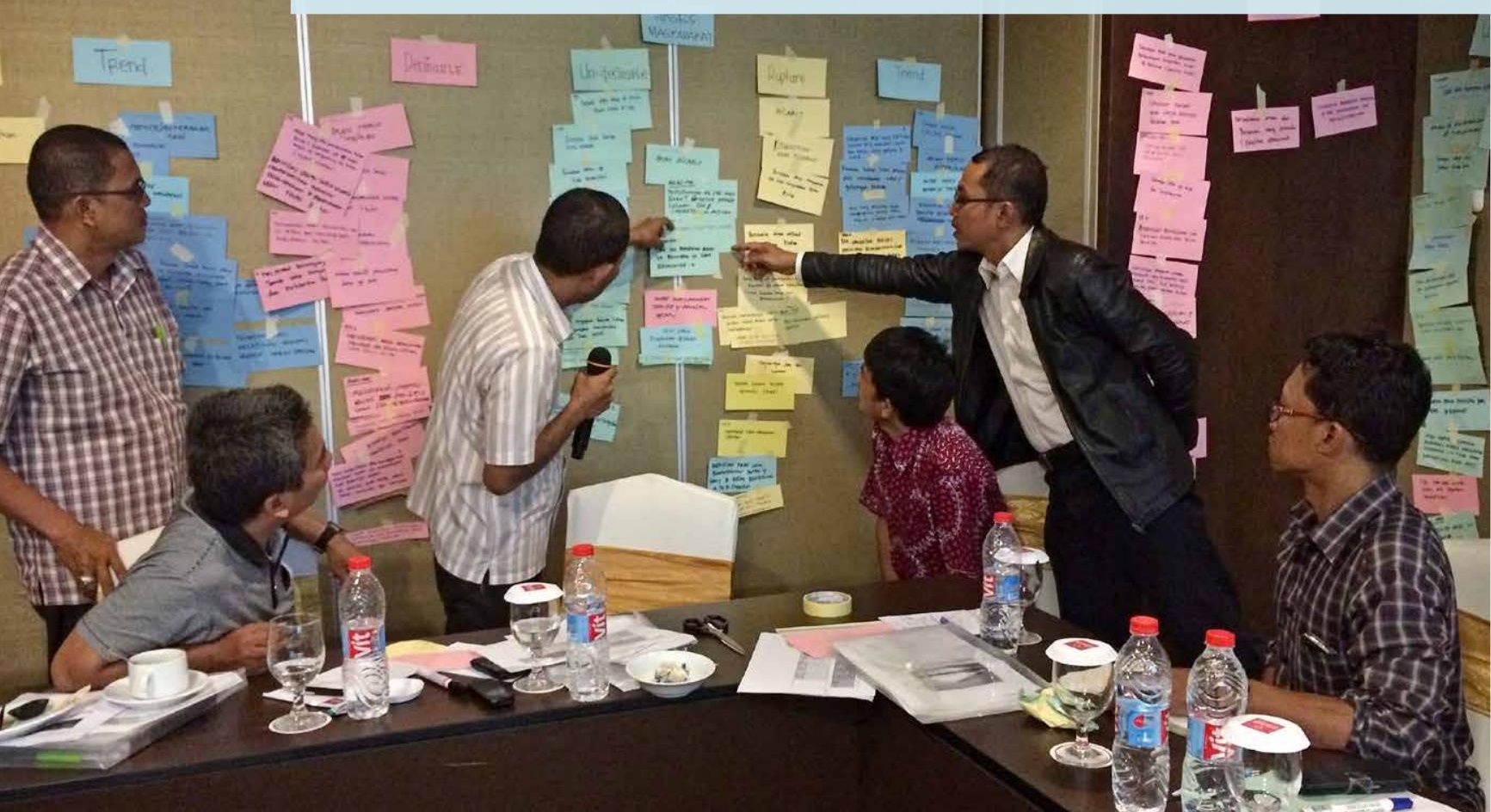




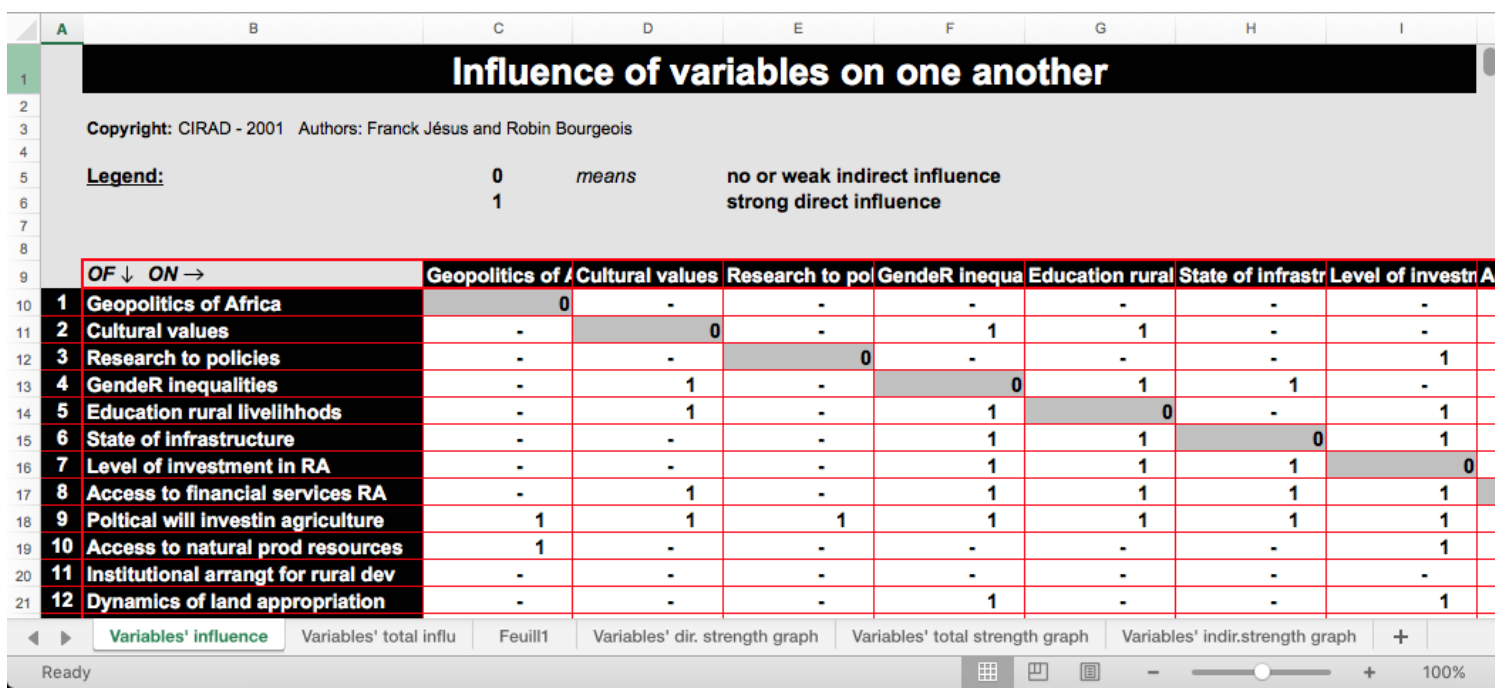

Figure 7. Feuille de travail exposant l'influence mutuelle des variables (facteurs).

\section{La feuille de travail « Variables' dir. strength graph »}

Cette feuille de travail présente un graphique dans lequel chaque facteur est situé le long de deux axes mesurant l'influence directe (axe vertical) et la dépendance directe (axe horizontal). Ce graphique permet de visualiser la position de chaque facteur et d'interpréter son rôle dans le système.

À cette fin, le graphique est divisé en quatre quadrants séparés par deux lignes en pointillé, l'une horizontale, l'autre verticale. Ce graphique se remplit automatiquement lorsque la matrice des influences directes est renseignée.

\section{La feuille de travail « Variables' indir. strength graph »}

De même, cette feuille comporte un graphique dans lequel chaque facteur se trouve le long de deux axes représentant l'influence indirecte et la dépendance indirecte. $\mathrm{Ce}$

\section{CONSEILS}

- Sauvegarder régulièrement la matrice sous différents noms au cours de l'analyse structurelle afin de toujours disposer d'une version de secours en cas de fausse manœuvre.

- Veiller à ce que la matrice finale soit stockée au moins sur trois disques durs indépendants. II ne faudrait pas perdre le fruit du travail de 20 personnes pendant trois jours intensifs ! 


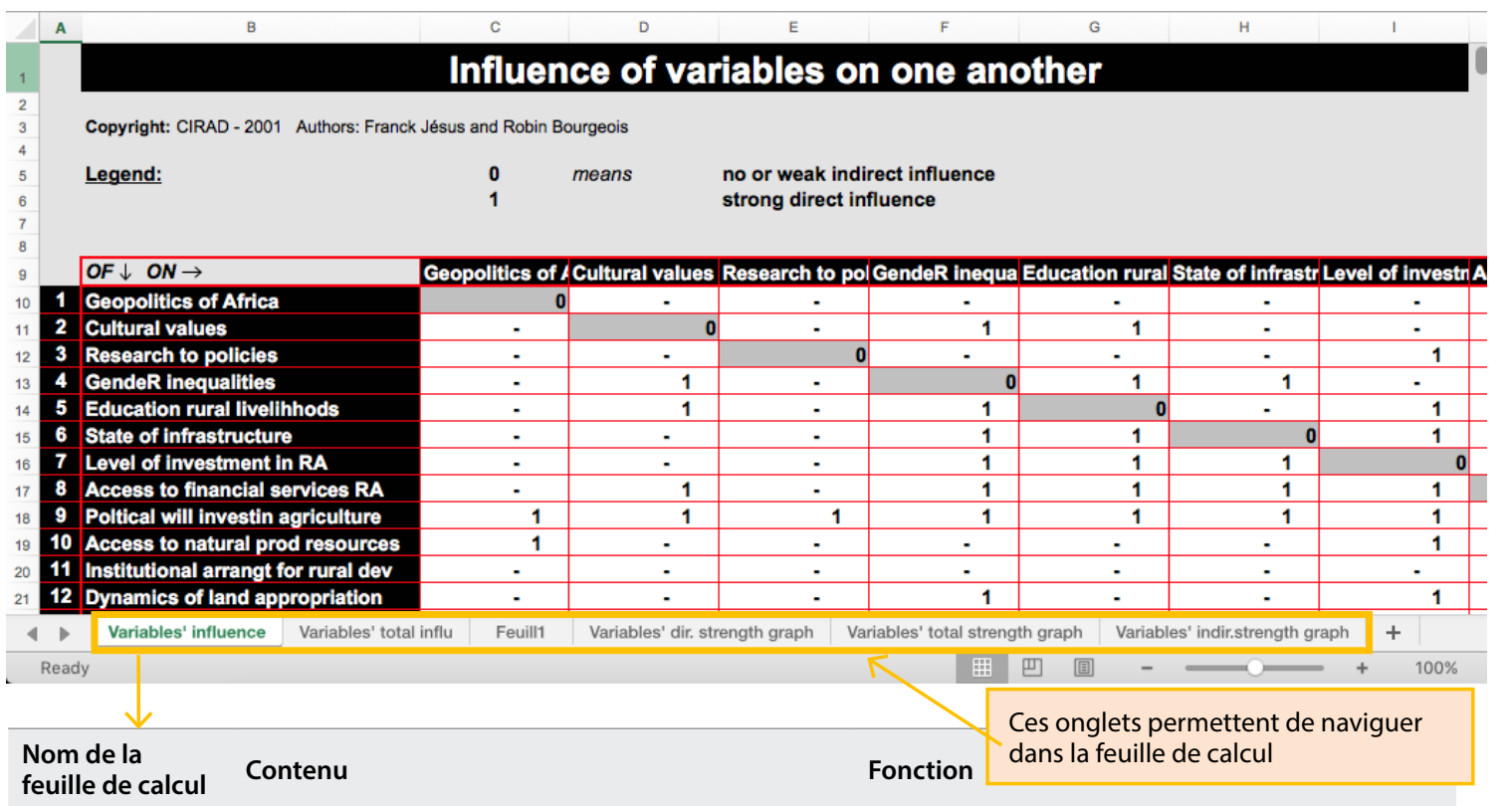

«Variables' Matrice dans laquelle sont entrés les noms des influence » facteurs et les valeurs de leur influence directe

Quatre tableaux situés en bas de la matrice pour exposer : I'influence directe, la dépendance directe, la force directe et la force directe pondérée de chaque facteur

« Variables' total influ »

"Feuill1"

«Variables' dir.

strength graph »

Matrice « Forces indirect influence on one another » dans laquelle les valeurs d'influence indirecte sont automatiquement calculées

Quatre tableaux situés en bas de la matrice pour exposer : I'influence indirecte, la dépendance indirecte, la force indirecte et la force indirecte pondérée de chaque facteur

Une matrice « Variables' total influence on one another » est située sous ces tableaux dans laquelle les valeurs directes et indirectes sont additionnées

Quatre tableaux situés en bas de la matrice pour exposer: I'influence globale, la dépendance globale, la force globale et la force globale pondérée de chaque facteur

Matrice à remplir manuellement

Graphique présentant la position de chaque facteur le long de deux axes selon leur influence directe et leur dépendance directe pondérées

«Variables' total Graphique présentant la position de chaque strength graph » facteur le long de deux axes selon leur influence totale et leur dépendance totale pondérées

«Variables' indir. Graphique présentant la position de chaque facteur strength graph » le long de deux axes selon leur influence indirecte et leur dépendance indirecte pondérées
Enregistre les valeurs d'influence directe et des données provenant d'autres matrices

Mesure le rôle direct de chaque facteur par trois indicateurs : dans quelle mesure il affecte le système, dans quelle mesure le système l'affecte et un classement correspondant à sa puissance relative

Produit les valeurs des influences indirectes en multipliant la première matrice par elle-même. Mesure le rôle indirect de chaque facteur par trois indicateurs : dans quelle mesure il affecte le système, dans quelle mesure le système l'affecte et un classement correspondant à sa puissance dans le système

Produit les valeurs d'influence totale en additionnant les valeurs directes et indirectes Évalue le rôle total de chaque facteur par trois indicateurs : dans quelle mesure il affecte le système, dans quelle mesure le système l'affecte et un classement correspondant à sa puissance dans le système

Utilise l'analyse des influences/dépendances indirectes aux niveaux plus hauts

Permet de visualiser la position des facteurs et détermine leur rôle actuel selon leur localisation dans les quatre quadrants

Permet de visualiser la position des facteurs et détermine leur rôle selon leur localisation dans les quatre quadrants du graphique

Permet de visualiser la position des facteurs et détermine leur rôle futur ou potentiel selon leur localisation dans les quatre quadrants

Figure 8. Résumé de la structure du logiciel. 
graphique permet de visualiser la position de chaque facteur et d'interpréter son rôle dans le système en fonction de son influence indirecte. À cette fin, le graphique est également divisé en quatre quadrants séparés par deux lignes en pointillé, l'une horizontale, l'autre verticale. Ce graphique se remplit automatiquement lorsque la matrice des influences directes est renseignée.

\section{La feuille de travail « Variables' total strength graph »}

Cette feuille comporte un graphique dans lequel chaque facteur se trouve le long de deux axes représentant l'influence totale (directe + indirecte) et la dépendance totale. Ce graphique permet de visualiser la position de chaque facteur et d'interpréter son rôle dans le système en fonction de son influence totale. Le graphique est divisé en quatre quadrants séparés par deux lignes en pointillé, l'une horizontale, l'autre verticale. Ce graphique se remplit automatiquement lorsque la matrice des influences directes est renseignée.

\section{La feuille de travail « Feuill1 »}

Dans cette feuille de travail, il faut remplir manuellement un tableau pour analyser les influences indirectes fortes entre les facteurs. Ce processus, qui est facultatif, contrôle la stabilité du système des influences indirectes et permet de repérer les problèmes relatifs aux facteurs.

\subsubsection{Utilisation du logiciel d'analyse structurelle : Compétences techniques}

Cette partie présente l'utilisation du logiciel en analyse structurelle de façon exhaustive et didactique.

\section{Entrer le nom des variables}

Avant d'entrer le nom des facteurs dans la matrice, affecter un acronyme à chacun, facile à comprendre et lisible dans les graphiques et les tableaux. Chaque acronyme est précédé d'un numéro pour l'identifier (voir le tableau 4 )

Dès que c'est fait, ouvrir le fichier Excel et choisir la première feuille de travail ("Variables' influence »). Positionner le curseur sur la première cellule de la colonne de couleur sombre sur la gauche de la matrice (cellule B11) et entrer le numéro et le nom du premier acronyme. Note : Un signe " - "st affecté à la cellule par défaut. Ne pas effacer ce signe. Le numéro et l'acronyme remplacent le signe " - ". Quand l'acronyme est entré dans la cellule, appuyer sur Entrée et aller à la cellule suivante du dessous. Répéter le processus jusqu'à ce que tous les acronymes soient entrés dans la première colonne de la matrice. 
À ce stade, on peut voir que les acronymes qui viennent d'être entrés se sont propagés dans les en-têtes des colonnes de la matrice, des autres matrices et de tous les autres tableaux de la matrice.

\section{Entrer des données dans la matrice}

Entrer les résultats de l'analyse des influences directes de chaque facteur horizontalement de gauche à droite, ligne par ligne. Entrer tous les résultats de l'analyse des influences directes du premier facteur sur tous les autres facteurs.

à la première ligne (ligne 11) de gauche à droite en commençant par la cellule D11 (qui concerne l'influence du facteur sur lui-même, égale à 0 par définition. Ne pas modifier les cellules diagonales grisées). Enregistrer et répéter l'opération pour le deuxième facteur, en commençant par la cellule $\mathrm{C} 12$ sur la gauche (sauf la D12, cellule de la diagonale en grisé), puis enregistrer. Répéter le processus jusqu'au dernier facteur de la matrice, enregistrer le résultat et faire une copie de sauvegarde du fichier.

La matrice est protégée. Cela signifie qu' on ne peut saisir que 0 ou 1 . Toute autre saisie déclenchera l'affichage d'un avertissement. Si l'on était sur le point de saisir des données erronées, fermer la fenêtre et entrer 0 ou 1 . Attention : toutes
Tableau 4. Variables d'une analyse structurelle.

\begin{tabular}{|c|c|}
\hline Acronyme & Numéro et nom du facteur \\
\hline 1. Cult_Norm & $\begin{array}{l}\text { 1. Normes et croyances } \\
\text { culturelles }\end{array}$ \\
\hline 2. R_Women & 2. Rôle des femmes \\
\hline 3. R_Youth & 3. Rôle des jeunes \\
\hline 4. P_Com_At & $\begin{array}{l}\text { 4. Attitude de la population/ } \\
\text { communauté }\end{array}$ \\
\hline 5.FTS_Gov & $\begin{array}{l}\text { 5. Gouvernance de la sécurité } d u \\
\text { foncier forestier }\end{array}$ \\
\hline 6. Pop_Press & 6. Pression démographique \\
\hline 7.Com_Cap & $\begin{array}{l}\text { 7. Capacités de gestion de la } \\
\text { communauté }\end{array}$ \\
\hline 8. FP_Stand & $\begin{array}{l}\text { 8. Normes en matière de produits } \\
\text { forestiers }\end{array}$ \\
\hline 9. Com_FTA & $\begin{array}{l}\text { 9. Sensibilisation de la } \\
\text { communauté au foncier forestier }\end{array}$ \\
\hline 10. S\&W_Ress & 10. Ressources en eau et du sol \\
\hline 11. For_Ress & 11. Ressources forestières \\
\hline 12. Acc_Land & 12. Accès à la terre \\
\hline 13. Acc_Fund & $\begin{array}{l}\text { 13. Accès à des ressources } \\
\text { financières }\end{array}$ \\
\hline 14. Acc_Input & 14. Accès aux intrants \\
\hline 15. Inc_Dist & 15. Répartition des revenus \\
\hline 16. FP_Price & 16. Prix des produits forestiers \\
\hline 17. Poverty & 17. Pauvreté \\
\hline 18. Ec_Int_FL & $\begin{array}{l}\text { 18. Intérêt économique des } \\
\text { terres boisées }\end{array}$ \\
\hline 19. Oil\&Gas & 19. Industrie pétrolière et gazière \\
\hline 20. For_Serv & 20. Paiement services forestiers \\
\hline 21. ForAg_Fund & $\begin{array}{l}\text { 21. Financement des agences } \\
\text { des forêts }\end{array}$ \\
\hline 22.Pol_Will & 22. Volonté politique \\
\hline 23.Pol_Inf & 23. Influence politique \\
\hline 24. LU_Pol & $\begin{array}{l}\text { 24. Politique d'utilisation des } \\
\text { terres }\end{array}$ \\
\hline 25. Land_Reg & 25. Enregistrement des terres \\
\hline 26. Imp_Strat & 26. Stratégies de mise en ceuvre \\
\hline
\end{tabular}


les cellules sont formatées par défaut avec la valeur 0 , qui apparaît comme «- ". Si l'on saisit des données erronées, qu'on les efface en laissant la cellule vide sans restaurer la valeur originale de 0 , cela empêchera le calcul automatique des influences indirectes. Le graphique des influences indirectes n'affichera aucune variable et le tableau des influences indirectes affichera des signes d'erreur. Si cela se produit, c'est qu'il y a au moins une cellule vide dans la matrice. Il faut la trouver pour corriger le problème. Par défaut, la valeur de toutes les cellules est de 0 . Par conséquent, tout problème dans la matrice des influences indirectes proviendra d'une erreur de saisie de données.

Les cellules $\mathrm{K} 7$ et L7 au-dessus de la matrice sont des cellules de contrôle qui signalent les cellules vides. Si la matrice comporte au moins une cellule vide, la L17 devient rouge et indique le nombre de cellules vides. Par ailleurs, toutes les cellules de la matrice ont été formatées pour devenir rouges si elles restent vides; cela permet de repérer aisément d'où viennent les problèmes signalés par la cellule L17. Grâce à ces cellules de contrôle, les influences indirectes peuvent être automatiquement calculées et les résultats peuvent s'afficher.

\section{Adapter la taille de la matrice}

La matrice des influences directes est un tableau de $100 \times 100$ cellules. Par défaut, seule une matrice de $73 \times 73$ cellules est visible, les autres lignes et colonnes étant masquées. Suivre les étapes ci-dessous pour adapter la taille visible de la matrice en fonction du nombre exact de facteurs entrés :

\section{Afficher les colonnes masquées}

Mettre le curseur en haut de la colonne BW. Cela met automatiquement en surbrillance la totalité de la colonne. Appuyer sur la touche Maj et déplacer le curseur sur la colonne de droite suivante qui est la colonne CZ. Ces deux colonnes sont maintenant en surbrillance. Faire un clic droit de la souris. Cliquer sur " Unhide » dans la boîte de dialogue qui apparaît. Toutes les lignes masquées entre BW et CZ devraient maintenant être visibles. Cliquer ensuite en haut de la colonne CX. En maintenant la touche Maj appuyée, déplacer le curseur vers la gauche jusqu’à sélectionner la première colonne sans en-tête ayant le nom d'un facteur. Faire un clic droit et cliquer sur " Hide " dans la boîte de dialogue. Toutes les colonnes entre la dernière colonne avec en-tête et la colonne CY devraient avoir disparu. Les valeurs de la colonne CX peuvent être encore plus visibles si l'on sélectionne la totalité de cette colonne pour changer la couleur de la police en noir. Les valeurs correspondant à l'influence directe totale de chaque facteur apparaitront plus clairement.

\section{Afficher les lignes masquées}

Répéter le même processus pour les lignes masquées situées entre les lignes 83 et 112 . Ensuite, masquer les lignes entre la 111 et la ligne suivant la dernière ligne sur laquelle se trouve le nom d'un facteur.

Ensuite changer la police de la ligne 111. 


\section{Adapter les tableaux des forces directes et indirectes}

Ces tableaux présentent les données nécessaires pour classer les facteurs selon leur force. Pour ce faire, copier le contenu du tableau des forces directes et indirectes dans un nouveau fichier Excel. D'abord, ouvrir un nouveau fichier Excel, le nommer (p. ex., ClassementFacteurs) et le sauvegarder dans le dossier où se trouve la matrice d'analyse structurelle. Puis sélectionner l'en-tête et tous les facteurs avec leurs valeurs correspondantes dans le tableau appelé weighted direct strength, qui part des cellules M114 et N114 vers le bas. Sélectionner les facteurs que vous avez saisis, mais pas toutes les cellules du tableau. Copier et coller dans le nouveau fichier, en sélectionnant l'option "Paste Values ». Répéter le processus pour le tableau situé dans la feuille de travail «Variable’s total influ » sous les cellules L109 et M109.

Pour classer les facteurs, sélectionner toutes les cellules du tableau weighted direct strength. Cliquer sur " Data » dans les choix proposés par le menu et sur "Sort». Cocher l'option "My data has headers ». Dans "Select by ", choisir le nom de la colonne qui présente les valeurs des facteurs. Enfin, sélectionner l'option permettant d'afficher les valeurs par ordre décroissant et cliquer sur « OK». Le tableau devrait maintenant afficher tous les facteurs dans un autre ordre en les classant du plus fort en haut au plus faible en bas. Répéter le processus pour le tableau des forces indirectes.

Les tableaux sont maintenant prêts pour comparer l'ordre des facteurs et leurs valeurs en fonction de leurs influences directes et indirectes.

Les facteurs en haut du tableau des influences directes doivent être considérés comme facteurs moteurs. S'ils se trouvent aussi en haut du tableau des influences indirectes, ils seront probablement des facteurs moteurs de poids. Les facteurs qui remontent beaucoup vers le haut, des influences directes aux influences indirectes, sont susceptibles d'influer sur l'avenir du système à long terme. Ceux qui décroissent de manière significative ont un impact plus immédiat.

\section{Comment utiliser les graphiques}

Tous les graphiques affichent automatiquement la position de chaque facteur en fonction de ses coordonnées d'influence et de dépendance. Ces coordonnées se trouvent sur les lignes immédiatement sur la gauche des tableaux se trouvant en bas de chaque matrice et qui affichent l'influence et la dépendance des facteurs.

Par défaut, tous les graphiques présentent des axes avec une échelle affichant des valeurs entre 0 et 2 . Avant d'analyser les graphiques, s'assurer que les facteurs dont les coordonnées d'influence et de dépendance supérieures à 2 s'affichent également. À cette fin, paramétrer le format des axes, vertical et horizontal, sur " automatic » pour la valeur maximale affichée. Faire un clic droit sur n'importe quelle valeur sur l'échelle de l'axe vertical. Une boîte de dialogue apparaît. Cliquer sur «Format axis ». Une autre boîte de dialogue apparait. Dans les options, où « Maximum » s'affiche, cocher "Automatic " puis fermer le graphique. Tout facteur dont la valeur d'influence est 
supérieure à 2 doit apparaitre dans le graphique. Répéter le même processus pour l'axe horizontal afin de faire apparaître tout facteur dont la valeur de dépendance est supérieure à 2 .

Enfin, comme la présentation du graphique a changé, déplacer manuellement les lignes horizontale et verticale en pointillé, qui marquaient à l'origine la valeur 1, afin qu'elles se trouvent de nouveau sur cette valeur. $\mathrm{Si}$, après avoir paramétré les deux axes sur le maximum, le graphique affiche des valeurs maximales inférieures à 2 (ce qui signifie qu'aucun facteur n'a une valeur supérieure à 2), revenir au paramétrage standard de la valeur maximale fixe de 2 . Les résultats gagneront ainsi en lisibilité.

Adapter au besoin la lisibilité des étiquettes des variables en cliquant une fois sur une étiquette pour sélectionner une police plus grande dans le menu Home.

\subsubsection{Mettre en lumière les facteurs moteurs}

Les facteurs moteurs sont les plus influents et puissants du système. On les appelle facteurs moteurs parce que tout futur changement orientera le système dans une certaine direction, souhaitable ou non. Ces facteurs étant les points d'ancrage des scénarios, leur identification est un point d'étape essentiel du processus d'élaboration.

Cette identification des facteurs moteurs repose sur deux opérations complémentaires : i) l'interprétation des graphiques qui permettent de visualiser la position de ces facteurs et ii) l'interprétation des tableaux qui présentent le calcul de l'influence, de la dépendance et de la force des facteurs.

Les participants sélectionnent les facteurs moteurs après avoir débattu et analysé ces deux interprétations, y compris les influences directes, indirectes et totales.

\section{Comment utiliser les graphiques}

Les graphiques d'influence (directe, indirecte, totale) exposent la répartition des facteurs dans quatre quadrants délimités par deux axes. Ces graphiques montrent la position de chaque facteur selon la valeur de son influence et de sa dépendance pondérées calculées à partir du tableau des influences et des dépendances. Les coordonnées de chaque facteur se trouvent dans la colonne de gauche des tableaux (colonnes $\mathrm{E}$ et $\mathrm{H}$ dans la feuille de travail «Variables' influence » et colonnes $\mathrm{D}$ et $\mathrm{G}$ dans la feuille de travail «Variables' total influ»). Elles sont cependant masquées pour une meilleure lisibilité des tableaux.

Les graphiques permettent de représenter l'espace où les facteurs peuvent être affichés à l'aide de leurs coordonnées d'influence et de dépendance. L'axe vertical est celui de l'influence et l'axe horizontal celui de la dépendance. Deux lignes en pointillé séparent les quatre quadrants, l'une verticale et l'autre horizontale. Ces lignes en pointillé se trouvent à la valeur 1 , qui représente le centre de la répartition des facteurs selon leur 
dépendance et leur influence. Par exemple, si le nombre total des influences dans le système est de 400 et qu'il y a 40 variables, la valeur moyenne d'un facteur est $400 / 40=10$. La valeur d'influence de chaque facteur est ensuite divisée par 10, ainsi un facteur moyen aura une valeur de 1 (c'est-à-dire la moyenne). Tous les facteurs dont la valeur est supérieure à 1 sont plus influents et tous ceux dont la valeur est inférieure à 10 sont moins influents. Ce calcul facilite la visualisation et l'exploitation des graphiques. Il ne change pas la forme du graphique. Les lignes en pointillé définissent quatre espaces différents appelés quadrants. Chaque quadrant correspond aux caractéristiques spécifiques des facteurs indiquées à la figure 9 .

La signification de chaque quadrant est la suivante :

- Quadrant supérieur gauche : facteurs moteurs*. Ces facteurs ont une influence supérieure à la moyenne et une dépendance inférieure à la moyenne.

- Quadrant supérieur droit : leviers Ils sont en même temps influents et dépendants. Ils peuvent agir sur le système, mais l'évolution du système peut aussi agir sur eux. Ils permettent d'accentuer la direction du système.

- Quadrant inférieur droit : résultats. Ils ont peu d'influence et sont très dépendants. D'autres facteurs dans le système déterminent leur configuration. Ils n'agissent pas sur lui.

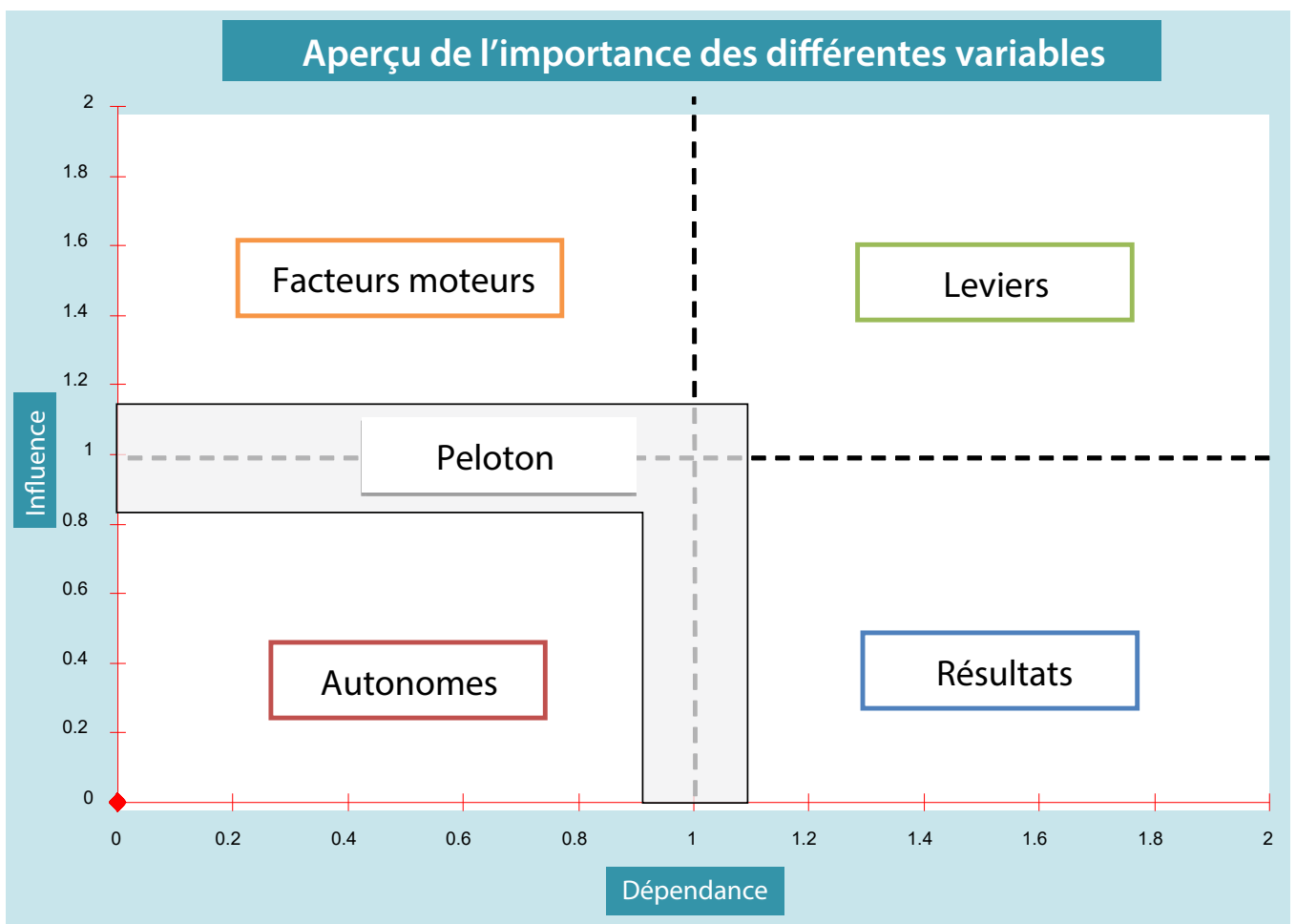

Figure 9. Aperçu de l'importance des différentes variables. 
- Quadrant inférieur gauche : autonomes. Ils se comportent plutôt indépendamment du système. Parfois, les autonomes correspondent à des problèmes que l'on croit être des facteurs déterminants, mais qui ne le sont pas. Il faut toutefois y réfléchir. Parfois, ils représentent aussi des facteurs qui ne sont pas encore suffisamment forts pour transformer le système, mais pourraient l'être à l'avenir (surtout s'ils se renforcent lors de l'analyse indirecte).

- Enfin, le "peloton" de facteurs se trouve en général dans la zone grise le long des axes, qui séparent le quadrant inférieur gauche des autres. Il n'est pas aisé d'identifier leur rôle dans le système. Ils contribuent à l'inertie du système.

\section{Utiliser les tableaux (influence directe, indirecte et totale)}

Les deux premiers tableaux en bas de chaque matrice (influence directe, indirecte et totale) mesurent les trois caractéristiques de chaque facteur : son influence, sa dépendance et sa force. Les valeurs calculées dans le tableau des influences correspondent à la somme des valeurs entrées sur la ligne relative à ce facteur dans la matrice. Le facteur ayant la valeur la plus élevée est le plus influent. De même, les valeurs de chaque facteur présentées dans le tableau des dépendances correspondent à la somme des valeurs entrées dans la colonne relative à ce facteur dans la matrice. Plus la valeur est élevée, plus le facteur est dépendant.

Dans le tableau 5 qui provient de la formation en Ouganda, les valeurs représentent la somme par ligne (influence) des nombres entrés dans la matrice. Il montre que le rôle de la jeunesse et celui de la gouvernance de la sécurité du foncier forestier ont l'influence la plus forte (les valeurs étant de 14 et 13). Les colonnes sur la droite indiquent la somme par colonne (dépendance) des valeurs entrées dans la matrice. Il montre que l'attitude de la population/communauté est le facteur qui subit l'influence la plus forte et qui est donc le plus dépendant (valeur 22). Les normes et croyances culturelles figurent parmi les facteurs les moins dépendants (valeur 3).

À droite de ces tableaux des influences et des dépendances, deux autres tableaux mesurent la force de chaque facteur. La force est le résultat de leur influence et de leur dépendance. C'est une seule mesure basée sur l'idée que deux facteurs ayant une influence similaire, mais des dépendances différentes, n'ont pas la même puissance dans le système. Le facteur ayant l'influence la plus forte et la dépendance la plus faible est plus puissant. La "force pondérée " utilise la même formule en classant les facteurs, leur répartition étant centrée sur la valeur « 1 ».

Avec le même exemple, le tableau 6 présente des valeurs représentant le résultat de l'influence et de la dépendance de chaque facteur. Dans la colonne de droite, cette valeur est ajustée pour que la répartition soit centrée sur 1 . Le facteur le plus fort ici a une force totale de 1,45 (gouvernance de la sécurité du foncier forestier). Le facteur le plus faible a une valeur de 0,32 (ressources en eau et celles du sol). 
Tableau 5. Direct influence versus direct dependence.

\begin{tabular}{lrlr}
\hline \multicolumn{1}{c}{ Influence directe } & \multicolumn{2}{c}{ Dépendance directe } \\
\hline 1. Cult_Norm & 9 & 1.Cult_Norm & 3 \\
2. R_Women & 10 & 2. R_Women & 12 \\
3. R_Youth & 14 & 3. R_Youth & 11 \\
4.P_Com_At & 12 & 4.P_Com_At & 22 \\
5. FTS_Gov & 13 & 5. FTS_Gov & 11 \\
6. Pop_Press & 11 & 6.Pop_Press & 18 \\
7. Com_Cap & 9 & 7.Com_Cap & 5 \\
8. FP_Stand & 6 & 8. FP_Stand & 3 \\
9. Com_FTA & 8 & 9.Com_FTA & 10 \\
10.S\&W_Ress & 5 & 10.S\&W_Ress & 11 \\
11. For_Ress & 10 & 11. For_Ress & 19 \\
12. Acc_Land & 7 & 12. Acc_Land & 12 \\
\hline
\end{tabular}

Tableau 6. Comparaison entre la force directe et la force directe pondérée.

\begin{tabular}{lllr}
\hline & Force directe & & \multicolumn{1}{c}{ Force directe pondérée } \\
\hline 1. Cult_Norm & 0.03 & 1. Cult_Norm & 1.39 \\
2. R_Women & 0.02 & 2. R_Women & 0.94 \\
3. R_Youth & 0.03 & 3. R_Youth & 1.61 \\
4.P_Com_At & 0.02 & 4.P_Com_At & 0.87 \\
5. FTS_Gov & 0.03 & 5. FTS_Gov & 1.45 \\
6. Pop_Press & 0.02 & 6. Pop_Press & 0.86 \\
7. Com_Cap & 0.02 & 7. Com_Cap & 1.19 \\
8. FP_Stand & 0.02 & 8. FP_Stand & 0.82 \\
9. Com_FTA & 0.02 & 9. Com_FTA & 0.73 \\
10. S\&W_Ress & 0.01 & 10.S\&W_Ress & 0.32 \\
11. For_Ress & 0.01 & 11. For_Ress & 0.71 \\
12. Acc_Land & 0.01 & 12. Acc_Land & 0.53 \\
\hline
\end{tabular}

\section{Choisir les facteurs moteurs}

Les facteurs moteurs sont ceux qui exercent l'influence la plus puissante du système. Ces facteurs peuvent influer directement sur le plus grand nombre d'autres facteurs. La sélection des facteurs moteurs commence avec le graphique de l'influence directe. Suivre les étapes indiquées ci-après : 


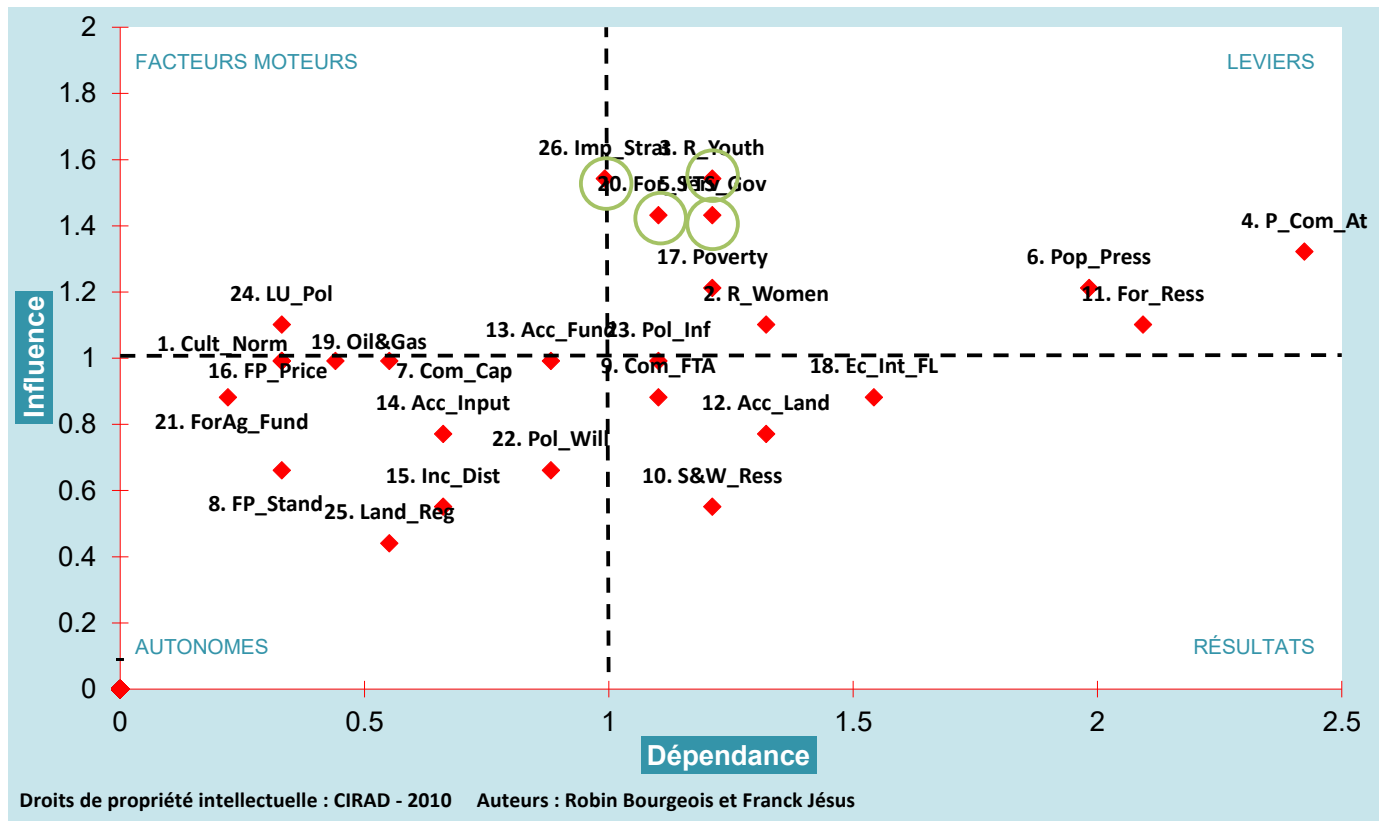

Figure 10. Exemple provenant de l'atelier de formation en Ouganda.

Note : Quatre facteurs ont d'abord été sélectionnés en raison de leur position en haut du graphique (en vert).

- $\quad$ Prendre le graphique de l'influence directe et observer les facteurs moteurs directs les plus forts, ceux se trouvant en haut du quadrant des " Facteurs » et ceux situés en haut à gauche du quadrant des "Leviers». Voir la figure 10.

- Étudier le tableau de l'influence directe pondérée pour voir le classement respectif des facteurs repérés dans le graphique. À cet effet, on peut copier et coller les valeurs du tableau et les classer par ordre décroissant. Un écart important entre les forces directes pondérées des facteurs peut permettre de séparer les facteurs les plus puissants des autres. Voir le tableau 7.

En prenant le même exemple, le tableau 7 classe les facteurs selon leur force directe pondér ée. Il montre que les quatre facteurs repérés se trouvent effectivement parmi les premiers. Cependant, il révèle aussi qu'un autre facteur (LU_Pol) a une force qui doit être prise en compte puisqu'il figure dans les premiers facteurs du tableau.

Le logiciel calcule automatiquement les influences indirectes (Figure 11). Les résultats servent à réfléchir au choix des facteurs moteurs et à mieux comprendre la structure du système et comment il peut évoluer. Les influences indirectes peuvent être interprétées comme indiquant les influences à long terme des facteurs. Un facteur devenant plus 
Tableau 7. Comparaison entre la force directe pondérée et la force indirecte pondérée.

\begin{tabular}{|c|c|c|c|}
\hline \multicolumn{2}{|c|}{ Weighted direct strength } & \multicolumn{2}{|c|}{ Weighted indirect strength } \\
\hline 26. Imp_Strat & 1.75 & 24. LU_Pol & 1.69 \\
\hline 3. R_Youth & 1.61 & 26. Imp_Strat & 1.65 \\
\hline 24. LU_Pol & 1.58 & 20. For_Serv & 1.50 \\
\hline 20. For_Serv & 1.51 & 3. R_Youth & 1.36 \\
\hline 5. FTS_Gov & 1.45 & 16. FP_Price & 1.35 \\
\hline 1. Cult_Norm & 1.39 & 19. OII\&Gas & 1.34 \\
\hline 16. FP_Price & 1.39 & 5. FTS_Gov & 1.33 \\
\hline 21. ForAg_Fund & 1.32 & 1. Cult_Norm & 1.31 \\
\hline 19. OIl\&Gas & 1.28 & 21. ForAg_Fund & 1.29 \\
\hline 7. Com_Cap & 1.19 & 17. Poverty & 1.14 \\
\hline 17. Poverty & 1.13 & 7. Com_Cap & 1.12 \\
\hline 13. Acc_Fund & 0.98 & 13. Acc_Fund & 1.08 \\
\hline 2. R_Women & 0.94 & 4. P_Com_At & 0.98 \\
\hline 23. Pol_Inf & 0.88 & 14. Acc_Input & 0.94 \\
\hline 4. P_Com_At & 0.87 & 9. Com_FTA & 0.91 \\
\hline 6. Pop_Press & 0.86 & 8. FP_Stand & 0.91 \\
\hline 8. FP_Stand & 0.82 & 6. Pop_Press & 0.88 \\
\hline 14. Acc_Input & 0.78 & 23. Pol_Inf & 0.79 \\
\hline 9. Com_FTA & 0.73 & 2. R_Women & 0.78 \\
\hline 11. For_Ress & 0.71 & 12. Acc_Land & 0.71 \\
\hline 18. Ec_Int_FL & 0.60 & 11. For_Ress & 0.67 \\
\hline 12. Acc_Land & 0.53 & 18. Ec_Int_FL & 0.58 \\
\hline 22. Pol_WHIl & 0.53 & 22. Pol_Will & 0.53 \\
\hline 15. Inc_Dist & 0.47 & 25. Land_Reg & 0.44 \\
\hline 25. Land_Reg & 0.37 & 15. Inc_Dist & 0.39 \\
\hline 10. S\&W_Ress & 0.32 & 10. S8W_Ress & 0.32 \\
\hline
\end{tabular}

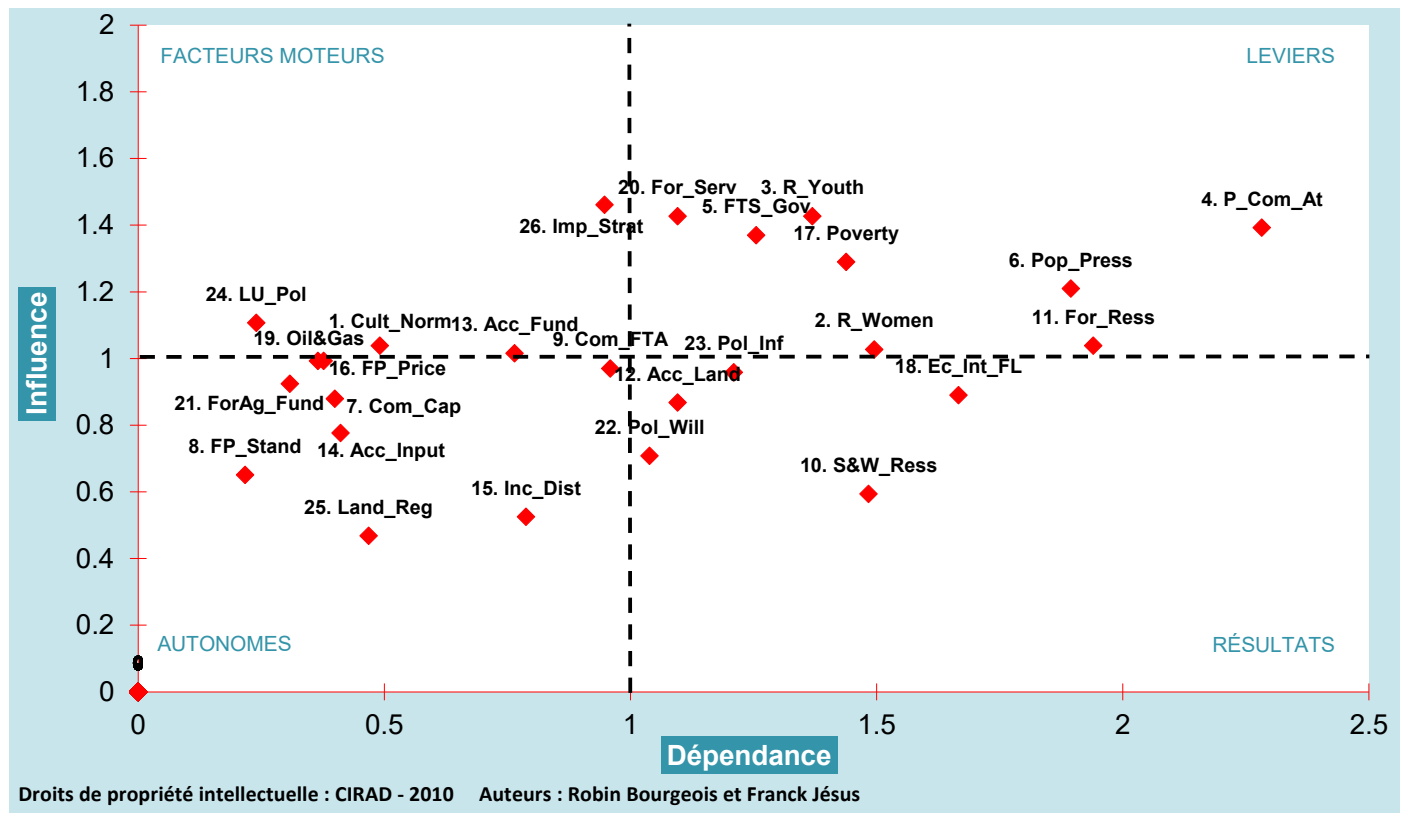

Figure 11. Graphique des influences indirectes. 


\section{Encadré 13. Exemple de regroupement de facteurs en un seul facteur moteur}

Au cours de la formation en Indonésie, après l'analyse des graphiques et des tableaux, les participants ont convenu de regrouper "Participation de la communauté » et «Institutions communautaires » en un seul facteur qui a été défini ainsi : "Rôle de la population et des organisations locales, notamment les communautés et les ONG, dans la gouvernance et la mise en œuvre de la réforme du foncier forestier ».

De même, " Gouvernance locale » et « Mise en cuvre des politiques » ont été regroupés en un seul facteur qui a été défini ainsi : "La gouvernance politique, économique et administrative de la mise en cuvre, par le gouvernement local, des politiques du gouvernement central concernant la réforme du foncier forestier ».

\section{Encadré 14. Choisir les facteurs : le cas du Pérou}

L'atelier de Loreto a décidé de regrouper les facteurs relatifs à un sujet similaire. Par exemple, nous avons regroupé "Capacité des fédérations autochtones », «Capacité des communautés autochtones », " Autonomisation des communautés autochtones » et « Organisation intercommunautaire » en un seul facteur baptisé « Autonomisation des communautés ». Les participants ont aussi décidé de déplacer certains facteurs considérés trop importants

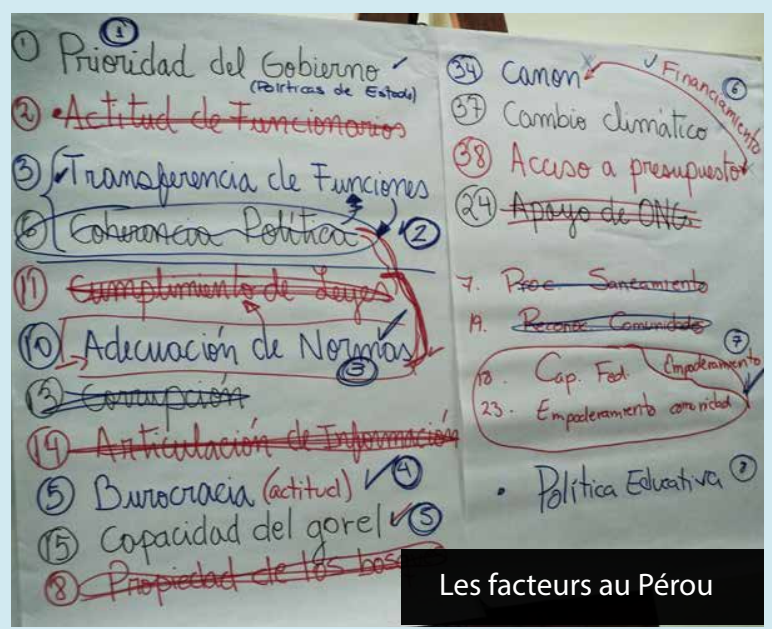
pour être rangés avec les leviers ou les résultats. Par exemple, «Qualité de l'éducation » a été renommée « Politique en matière d'éducation » puis déplacée des résultats vers les facteurs moteurs.

\section{Enseignements :}

Les participants n'ont sélectionné que quelques facteurs moteurs pour élaborer les scénarios. Par conséquent, s'il y a deux facteurs ( $A$ et $B$ ) et que $A$ influence $B$, il faut choisir A. Regrouper les facteurs similaires parce que l'action prévue pour l'un sera la même pour l'autre.

Voici le tableau final des facteurs moteurs que nous avons sélectionnés. D'abord, nous avons écrit tous les facteurs apparaissant dans le quadrant « Facteurs moteurs » du graphique d'influence. Nous avons regroupé certains d'entre eux, en avons éliminé d'autres (ceux qui sont influencés par un autre facteur déjà choisi) et en avons ajouté d'autres provenant d'autres quadrants. 
influent grâce à son influence indirecte est réputé avoir potentiellement un impact à plus long terme. Ceci parce qu'une influence indirecte opère par d'autres facteurs « intermédiaires » et se déclenche à l'issue d'un délai plus long.

Si un facteur appartenant au quadrant « Leviers » du graphique des influences directes se déplace vers le quadrant " Facteurs » du graphique des influences indirectes, par exemple, il devrait être pris en compte lors de la sélection des facteurs moteurs (Figure 11).

Dans cet exemple de l'Ouganda, le graphique des influences indirectes n'indique pas d'évolution importante dans la position des facteurs. De même, les premiers facteurs dans le tableau des forces directes et indirectes pondérées restent identiques. Cela confirme que le rôle des facteurs moteurs sélectionnés est à la fois immédiat et à long terme dans le système.

Pour la sélection finale des facteurs moteurs, le nombre de facteurs choisis doit être équilibré. Parce que les facteurs moteurs serviront à bâtir les scénarios, plus ces facteurs sont nombreux, et plus le processus d'élaboration des scénarios sera compliqué. À l'inverse, s'il y a trop peu de facteurs, cela induira une trop grande simplification de l'exploration* du futur.

Ce serait gâcher la masse d'informations produites lors des étapes précédentes.

D'expérience, certains principes peuvent être utilisés à cette fin :

- Dans la plupart des cas, les scénarios sont élaborés avec quatre à huit facteurs.

- Il est toujours possible d'adapter le nombre de facteurs en tenant compte de la diversité des configurations futures plausibles (voir l'étape suivante). Si certains facteurs présentent un nombre limité de configurations plausibles, on peut sélectionner plus de facteurs.

- Il est possible d'envisager de regrouper certains facteurs en un seul si les participants pensent qu'il y a une bonne raison de le faire.

\section{4 Étape 4 : Élaborer les scénarios}

Cette étape commence par l'identification du type de transformation qui pourrait toucher à l'avenir chaque facteur moteur sélectionné. Chaque facteur moteur peut évoluer dans des directions différentes induisant une configuration précise à l'avenir. Ces configurations servent ensuite à produire des scénarios plausibles. Un scénario n'est ni une prédiction ni une prévision*. C'est l'anticipation* d'une transformation plausible par l'exploration de voies alternatives. Lors de l'élaboration des scénarios, cette exploration doit être suffisamment ample pour permettre l'identification de plusieurs avenirs plausibles et contrastés. 


\subsubsection{Définir les configurations des facteurs moteurs}

Cette activité, parfois appelée " analyse morphologique ", vise à identifier différents avenirs cohérents, pertinents et plausibles. Pour chaque facteur moteur précédemment sélectionné, les experts détermineront les configurations possibles de chacun à l'avenir, et surtout les configurations contrastées et incompatibles. Une configuration est une hypothèse concernant l'évolution possible d'un facteur ; elle décrit le facteur dans l'avenir à l'horizon temporel retenu ; il s'agit d'une description qualitative*, et pas seulement d'une mesure quantitative* de ce facteur. Plusieurs configurations sont considérées, y compris les ruptures. Pour chaque facteur, les configurations doivent être contrastées et incompatibles, ce qui veut dire que deux configurations ne peuvent être simultanées.

Par exemple, au cours de l'atelier au Pérou, un des facteurs moteurs choisis était «Relations entre les usagers des forêts ». Pour ce facteur, quatre configurations contrastées ont été identifiées :

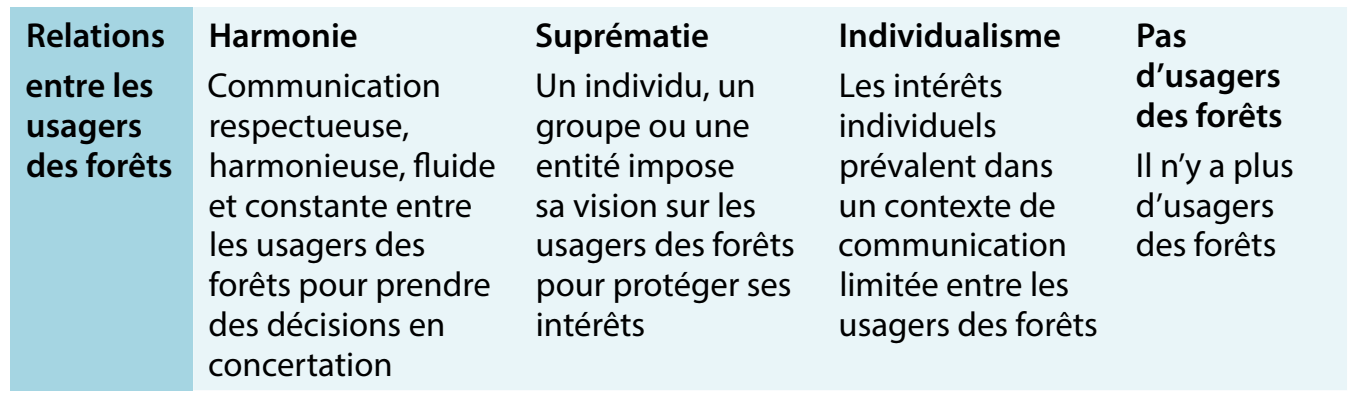

Dans cet exemple, les situations futures décrites sont contrastées et incompatibles avec les autres. La quatrième configuration est une rupture par rapport à la situation actuelle. La première représente une configuration souhaitable pour tous; la deuxième et la troisième sont considérées non souhaitables, mais plausibles et on les observe déjà dans une certaine mesure.

Lors du processus d'identification des configurations futures, les participants sont donc censés proposer des configurations en rupture avec le passé ou le présent. C'est un élément essentiel qui est rarement pris en compte dans la démarche prospective. Le débat sur les configurations suit un processus interactif, facteur après facteur, avec échange d'idées pour aboutir à un accord sur leur sélection finale. Les configurations qui sont trop improbables ne doivent pas être retenues. Il faut cependant être prudent avant d'affirmer que quelque chose est improbable. 


\section{Procédure :}

1. Tracer un tableau en inscrivant les facteurs sélectionnés dans la première colonne et en prévoyant d'autres colonnes pour les configurations. Présenter le tableau sur un support vertical.

2. Commencer par le premier facteur et travailler avec les participants sur l'identification de ses configurations futures de la manière suivante :

a. Distribuer une fiche de couleur à chaque participant (p. ex., rose) lors d'un premier tour. Leur demander d'écrire une configuration souhaitable de ce facteur à l'avenir (c'est-à-dire à l'horizon temporel convenu). Ramasser les fiches et délibérer afin d'en dégager un nombre de configurations contrastées et incompatibles. Pour cela, utiliser un support vertical pour exposer les fiches et les réarranger.

b. Procéder à un deuxième tour en distribuant aux participants une autre fiche de couleur (p. ex., gris), en leur demandant cette fois de penser à des configurations non souhaitables du même facteur. Ramasser les fiches et délibérer comme précédemment jusqu'à parvenir à un accord sur les configurations futures.

c. Procéder à un troisième tour avec les participants pour les aider à être créatifs en imaginant les configurations futures. Leur demander d'imaginer d'autres situations qui n’ont pas encore été envisagées, souhaitables ou non. De même, ramasser les fiches et délibérer comme précédemment. Ce troisième tour n'est pas obligatoire, car il n'est pas forcément possible d'envisager d'autres configurations qui seraient différentes et contrastées par rapport à celles déjà identifiées. Ce tour peut aussi être facultatif si un nombre suffisant de configurations contrastées a déjà été évoqué lors des deux premiers tours.

Tableau 8. Les facteurs moteurs au Pérou.

\begin{tabular}{|c|c|c|c|c|c|}
\hline & 1 & 2 & 3 & 4 & 5 \\
\hline $\begin{array}{l}\text { A. Système } \\
\text { éducatif }\end{array}$ & $\begin{array}{l}\text { Éducation } \\
\text { pourtous }\end{array}$ & $\begin{array}{l}\text { Enseignement } \\
\text { régionalisé }\end{array}$ & $\begin{array}{l}\text { Enseignement } \\
\text { privé }\end{array}$ & $\begin{array}{l}\text { Enseignement } \\
\text { défaillant }\end{array}$ & $\begin{array}{l}\text { Éducation } \\
\text { personnelle }\end{array}$ \\
\hline $\begin{array}{l}\text { B. Relations } \\
\text { entre les } \\
\text { usagers des } \\
\text { forêts }\end{array}$ & Harmonie & Suprématie & Individualisme & $\begin{array}{l}\text { Plus d'usagers } \\
\text { des forêts }\end{array}$ & \\
\hline $\begin{array}{l}\text { C. Autres } \\
\text { usages de la } \\
\text { forêt }\end{array}$ & $\begin{array}{l}\text { Biens et } \\
\text { services } \\
\text { publics }\end{array}$ & Extraction & Fluctuations & & \\
\hline $\begin{array}{l}\text { D. Valeurs des } \\
\text { décideurs }\end{array}$ & $\begin{array}{l}\text { Bien } \\
\text { commun }\end{array}$ & Corruption & Indifférence & Incertitude & \\
\hline $\begin{array}{l}\text { E. Processus } \\
\text { décisionnel }\end{array}$ & Inclusion & Centralisation & $\begin{array}{l}\text { Autoritarisme } \\
\text { des pouvoirs }\end{array}$ & Niveau & \\
\hline
\end{tabular}


3. Écrire un nombre raisonnable de configurations (2 à 6) qui sont contrastées et incompatibles dans les colonnes correspondantes du tableau.

4. Répéter cette opération pour tous les facteurs du tableau (voir le tableau 8, qui est un tableau synthétique de l'atelier de formation au Pérou).

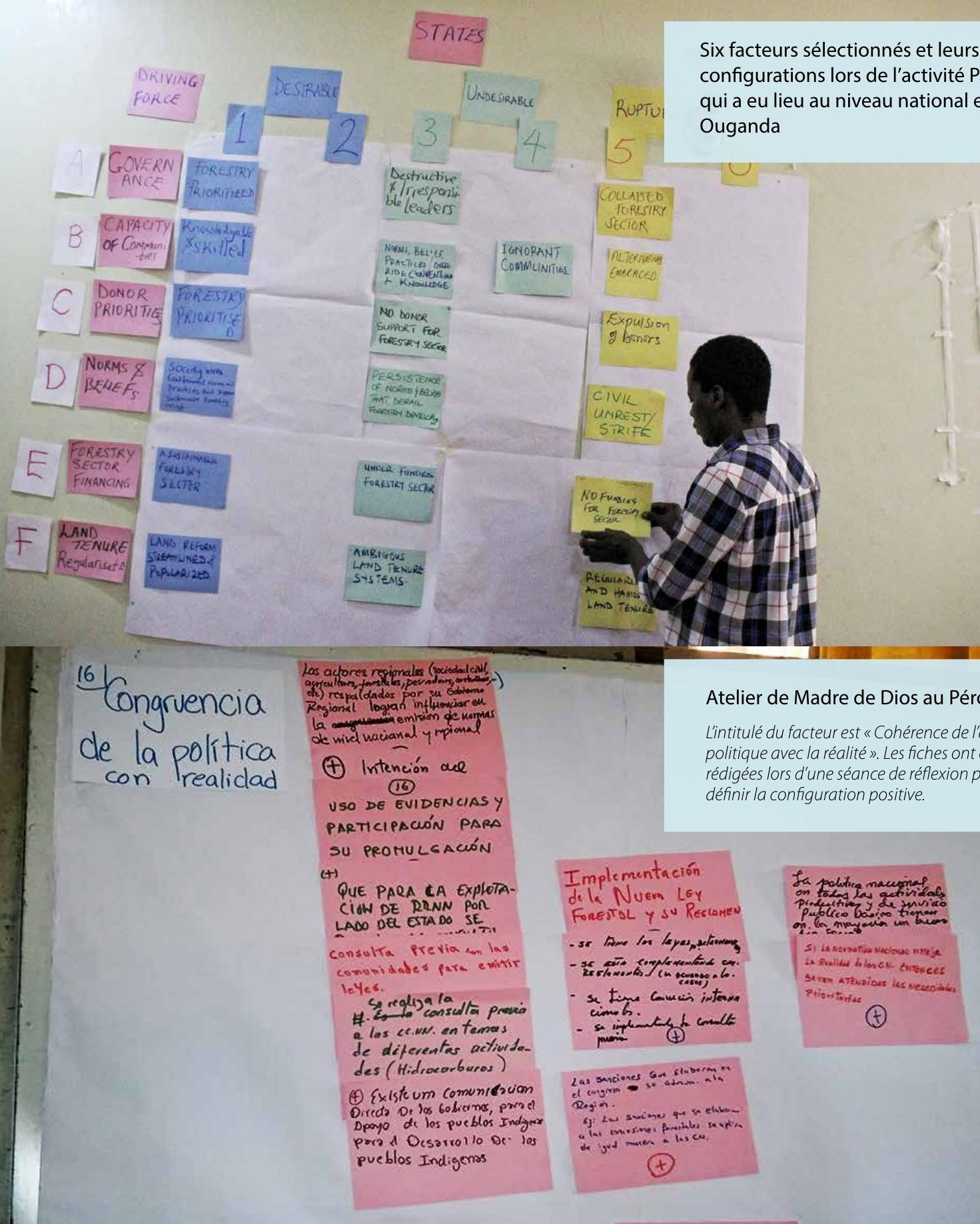




\section{CONSEILS}

Exemple de réflexion sur les configurations des facteurs de changement. Cela concerne 4 facteurs de changement sur 8 (H. Politique en matière d'éducation, A. Priorité du gouvernement, B. Transfert de fonctions, $C$. Adapter la réglementation à la réalité locale, voir les fiches de couleur jaune sur la photo ci-dessus).

- Le débat sur les configurations n'est pas toujours facile. Souvent, les fiches ne décrivent pas exactement une configuration future, mais portent une affirmation d'ordre général. Elles indiquent aussi parfois une information sur les causes ou les conséquences d'une configuration sans décrire celle-ci. Pour guider le débat :

a. Disposer les fiches verticalement, en mettant les plus générales en haut (à partir de ces fiches, on peut trouver un titre caractérisant les configurations comme, par exemple, les mots en gras du tableau 8). Mettre dessous les fiches qui décrivent les causes de la future configuration. Puis dessous, placer les fiches qui décrivent plus précisément la future configuration (c'est l'information vraiment nécessaire). Encore en dessous, mettre les fiches qui décrivent les conséquences de la configuration.

b Disposer les fiches de façon logique, pour relier verticalement les causes, la description des configurations et les conséquences correspondantes.

c. Séparer les configurations contrastées (les fiches du milieu) pour définir celles qui sont incompatibles.

- Quand les participants sont d'accord sur les différentes configurations, les inscrire sur le tableau et noter les causes et les conséquences séparément pour conserver ces informations pour les étapes suivantes.

Atelier de Loreto au Pérou

La photo montre les quatre facteurs sélectionnés sur les huit, avec leurs configurations.

$$
\begin{aligned}
& \text { H. Politica } \\
& \text { Educativa. }
\end{aligned}
$$

A. Prioridad del Gobierno

(1) Central/Regional

B. Transferenca de funciones

$$
\begin{aligned}
& \text { C. Adecuación de } \\
& \text { hormas a la } \\
& \text { realidad. }
\end{aligned}
$$
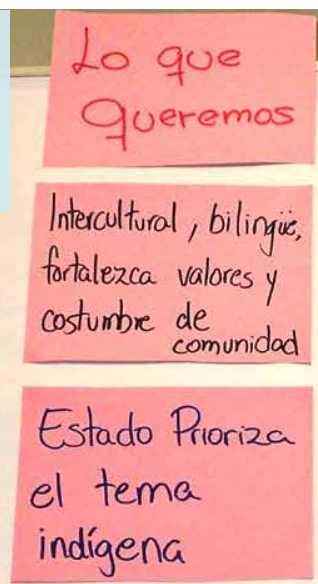

Coordinación efectiva del gobierno regional y local

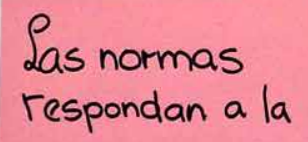

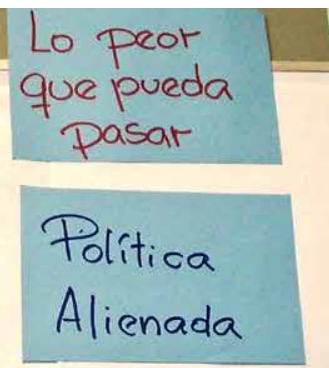

Esta Prioriza act. minera/petro/ agroindus

Centralismo REALIDAD Educación centralista

Estado tiene marco legislativo para CCNN pero no se cumple por $i$ econo, buro, corr.

\section{Transferencia de funciones NO es efectiva no coherencia}

No existe

normativa relao Normativa legal Inconsul 


\subsection{2 Élaborer les scénarios}

Un scénario est la description d'une possible situation future en fonction d'un ensemble explicite, cohérent et logique d'hypothèses sur les principales relations et les facteurs. Le processus d'élaboration des scénarios repose ici sur des hypothèses émises grâce à l'exploration des configurations futures plausibles des facteurs qui a eu lieu lors de l'étape précédente. Un scénario est une association plausible de facteurs à des configurations différentes.

Le nombre total de scénarios potentiels est obtenu en multipliant le nombre de facteurs par le nombre de leurs configurations. Dans le tableau 8 qui concerne le Pérou, aux cinq facteurs correspondaient respectivement cinq, quatre, trois, quatre et quatre configurations. Le nombre total de scénarios serait dans ce cas : $5 \times 4 \times 3 \times 4 \times 4=960$ scénarios.

Cependant, le nombre de scénarios plausibles peut être réduit en vérifiant la compatibilité entre les différentes configurations. Pour ce faire, dresser la liste des configurations qui ne peuvent pas coexister. Deux configurations sont incompatibles si les futurs correspondants ne peuvent pas coexister sur un plan logique et plausible.

Pour faciliter l'identification des configurations incompatibles, affecter à chaque facteur un code de référence (p. ex., une lettre capitale) et à chaque configuration un nombre. Les experts doivent réfléchir aux configurations qui ne sont pas compatibles en utilisant les codes prévus avec les lettres et les nombres. Dans le cas du Pérou, 26 incompatibilités ont été identifiées :

\section{A1/B2 A1/B3 A1/C2 A1/D2 A1/D3 A1/E2 A1/E3 A2/B3 A2/E2 A3/B1 A3/D1 A3/E1 \\ B1/C2 B1/C3 B1/D2 B1/D3 B1/E2 B1/E3 B2/D4 B2/E1 B2/E4 B3/D1 B3/E1 B4/C1 D1/E2 D2/E1}

Ceci signifie, par exemple, que la configuration décrite à la cellule B1 (à l'intersection de la ligne $\mathrm{B}$ et de la colonne 1) ne peut pas coexister avec la configuration inscrite à la cellule D2 (ligne D et colonne 2). En effet, les relations harmonieuses entre les parties prenantes qui utilisent les forêts ne sont pas compatibles avec les valeurs de décideurs animés par l'appât du gain et la corruption. Un scénario qui associerait ces deux configurations ne serait pas plausible, car sans cohérence interne. Par conséquent, on écartera tout scénario présentant cette association ou toute association indiquée ci-dessus dans le cas du Pérou. La méthode est efficace pour réduire le nombre de scénarios possibles en évitant de choisir des scénarios incohérents.

\section{Créer des scénarios}

La synopse d'un scénario est une combinaison plausible des configurations des facteurs. Par exemple, dans le tableau 9, un scénario plausible est représenté par la combinaison 
Tableau 9. Guide d'utilisation du logiciel pour évaluer les scénarios

\section{Entrer les incompatibilités}

Ouvrir le logiciel. Dans la première colonne de la feuille de travail « Incompatibilities », écrire toutes les incompatibilités identifiées (en commençant à la cellule A10) en suivant cet exemple: A1/B2, signifiant que la configuration 1 du facteur A est incompatible avec la configuration 2 du facteur $B$.

Par défaut, la première colonne est formatée pour accepter 150 incompatibilités. S'il y en a moins, il n'y a pas besoin de modifier le tableau. S'il y en a plus, le tableau peut être agrandi en copiant la dernière ligne (ligne 159) autant de fois que nécessaire.

\section{Indiquer le nombre de configurations}

La première ligne appelée « Variables » comporte huit cellules de $\mathrm{A}$ à $\mathrm{H}$, correspondant à un nombre maximal de huit facteurs. Comme signalé plus haut, le nombre maximal recommandé pour le processus d'élaboration de scénarios est de huit facteurs. S'il y en a moins (p. ex., cinq facteurs), ils apparaîtront dans le tableau de A à E.

À la deuxième ligne appelée "No. of states », entrer le nombre exact de configurations identifiées dans le tableau des configurations futures sous la lettre de chaque facteur se trouvant sur la ligne 1. Par exemple, s'il y a cinq facteurs dont la configuration future a été notée $2,5,3,3$ et 4 , entrer sur la ligne 2 le nombre 2 sous la lettre $A$ située à la ligne 1 , puis 5 sous $B, 3$ sous $C, 3$ sous $D$ et 4 sous $E$. Cela évitera, à l'étape suivante, d'entrer pour une configuration un nombre supérieur au nombre admissible (ce serait alors une configuration qui n'existerait pas).

\section{III. Évaluer les scénarios}

Quand les étapes ci-dessus sont accomplies, le logiciel est prêt à évaluer instantanément la plausibilité de n'importe quel scénario. Pour ce faire, il compare toutes les combinaisons de configurations dans les scénarios avec toutes les combinaisons incompatibles.

D'abord, à la ligne 3 appelée « Enter scenario », entrer le nombre de configurations correspondant à chaque facteur du scénario. Par exemple, si un scénario est caractérisé « A1 B3 C2 D3 E4 », entrer respectivement les chiffres 1, 3, 2, 3 et 4 dans les cellules B3, C3, D3, E3 et F3. Si l'on entre un chiffre erroné, c'est-à-dire supérieur au nombre de configurations, ou un signe qui n'est pas un chiffre, un signal d'erreur apparaîtra dans la cellule située sous l'erreur à la ligne 4. Annuler l'action et entrer le bon chiffre.

Deuxièmement, quand tous les chiffres correspondant aux configurations du scénario sont entrés, le scénario sera ou non accepté :

Résultat 1 : Si la cellule A7 reste bleue et que toutes les cellules de la ligne 7 restent vertes, le scénario est acceptable.

Résultat 2 : Si la cellule A7 devient rouge, le scénario envisagé présente des combinaisons incompatibles. Les cellules rouges de la ligne 7 afficheront le détail de la combinaison incompatible. Cela signifie que ce scénario doit être écarté.

Répéter le processus d'évaluation pour tous les scénarios suivants jusqu'au dernier. 
des configurations A3-B3-C3-D3-E2. Ce scénario s'appelle " Moi d'abord " (voir l'encadré 15).

Le fait de représenter chaque scénario par un code constitué de lettres et de chiffres facilite leur regroupement et leur tri. Cela permet aussi d'écarter les scénarios redondants, de les regrouper et de délibérer sur les résultats, et en particulier sur l'identification des représentations contrastées de l'avenir. À ce stade, le processus d'élaboration des scénarios permet d'aboutir pour chaque scénario à une combinaison codée (voir les photos ci-dessous).

Grâce à ce processus, il est possible d'identifier un grand nombre de scénarios. Les ateliers de formation sur l'avenir de la sécurité du foncier forestier ont permis de définir en moyenne plus de 10 scénarios. Après délibération et approfondissement, ce nombre a été réduit à sept ou huit scénarios. Le nombre de scénarios n'est pas habituellement prédéfini. Il dépend de la situation.

Les membres du groupe d'experts souhaitent parfois définir à l'avance le nombre de scénarios. En général, ce n'est pas conseillé. Mais si les membres choisissent de le faire, ils devraient travailler sur au moins cinq scénarios. Cela permettra aux experts d'exercer leur créativité et de ne pas se limiter à la pratique habituelle qui consiste à définir trois scénarios (un positif/souhaitable, un neutre/tendance*/statu quo et un négatif/défavorable).

\section{CONSEILS}

- Identifier de façon systématique et exhaustive les incompatibilités entre les configurations des facteurs. Pour ce faire, observer d'abord la cellule $A 1$ et vérifier si les configurations marquées aux lignes du dessous (en commençant par la ligne B) sont incompatibles avec celle figurant en $A 1$. Puis passer à $A 2$ et ainsi de suite. Quand la ligne A est terminée, passer à la B1. Répéter le processus en partant de la ligne $C$ (pas besoin de le refaire pour la ligne A). Répéter ces actions jusqu'à l'avant-dernière ligne du tableau où nous terminons l'analyse en la comparant à la dernière ligne.

- Utiliser le logiciel fourni en suivant les explications ci-dessous.

\section{Encadré 15. Le scénario « Moi d'abord » au Pérou}

Le système éducatif est privé, onéreux, exclusif et discriminatoire. Il est fondé sur des valeurs appartenant à des réalités étrangères au pays. Les connaissances locales ne sont pas prises en compte, et la participation des enfants et les jeunes est rare. Entre les usagers des forêts, les intérêts personnels et l'absence de communication prévalent. Les processus décisionnels sont verrouillés, centralisés, discriminatoires, sans participation de la population, et influencés par les intérêts de groupes d'acteurs puissants. Les décideurs sont indifférents à la forêt, aux services qu'elle procure et à ses populations, dont ils n'apprécient pas la valeur. L'exploitation incontrôlée des ressources forestières et minières est la norme. 


\section{Procédure}

1. Disposer le tableau présentant toutes les configurations des facteurs sur un support vertical bien visible de tous les participants. Distribuer le même tableau à chacun pour qu'il l'utilise directement pour créer des scénarios.

2. Commencer par un premier tour en demandant à chaque participant de créer un scénario souhaitable de la manière suivante :

a. Distribuer une fiche de couleur à chaque participant (p. ex., rose). Leur demander d'écrire une combinaison de configurations correspondant à une future situation du système qu'ils considèrent souhaitable. Au bout de quelques minutes, ramasser toutes les fiches.

b. Evaluer toutes les combinaisons proposées à l'aide du logiciel «Incompatibilities». Écarter les scénarios qui présentent des configurations incompatibles. Ne conserver que les scénarios plausibles. Placer toutes les fiches restantes sur un support vertical où on peut les afficher et les réarranger.

c. Lors de l'affichage des fiches, éliminer celles qui font double emploi (c'està-dire celles qui indiquent la même combinaison). Regrouper les fiches en fonction de leurs similitudes (celles qui présentent un grand nombre de configurations similaires).

d. Délibérer sur les scénarios qui ne diffèrent que sur un petit nombre de configurations, en veillant à garder les scénarios qui sont les plus contrastés et les plus cohérents. Cela garantira que les scénarios finalement sélectionnés représenteront des situations très différentes. À cette fin, quand le groupe est d'accord sur un scénario contrasté, écarter les autres qui se rapprochent de celui-ci sans être aussi contrastés. Pour ce faire, lire tout haut aux participants le contenu du scénario (qui est la combinaison des codes). Leur demander de citer la combinaison la plus plausible et la plus contrastée.

e. Garder les scénarios "souhaitables » sur un côté du support vertical.

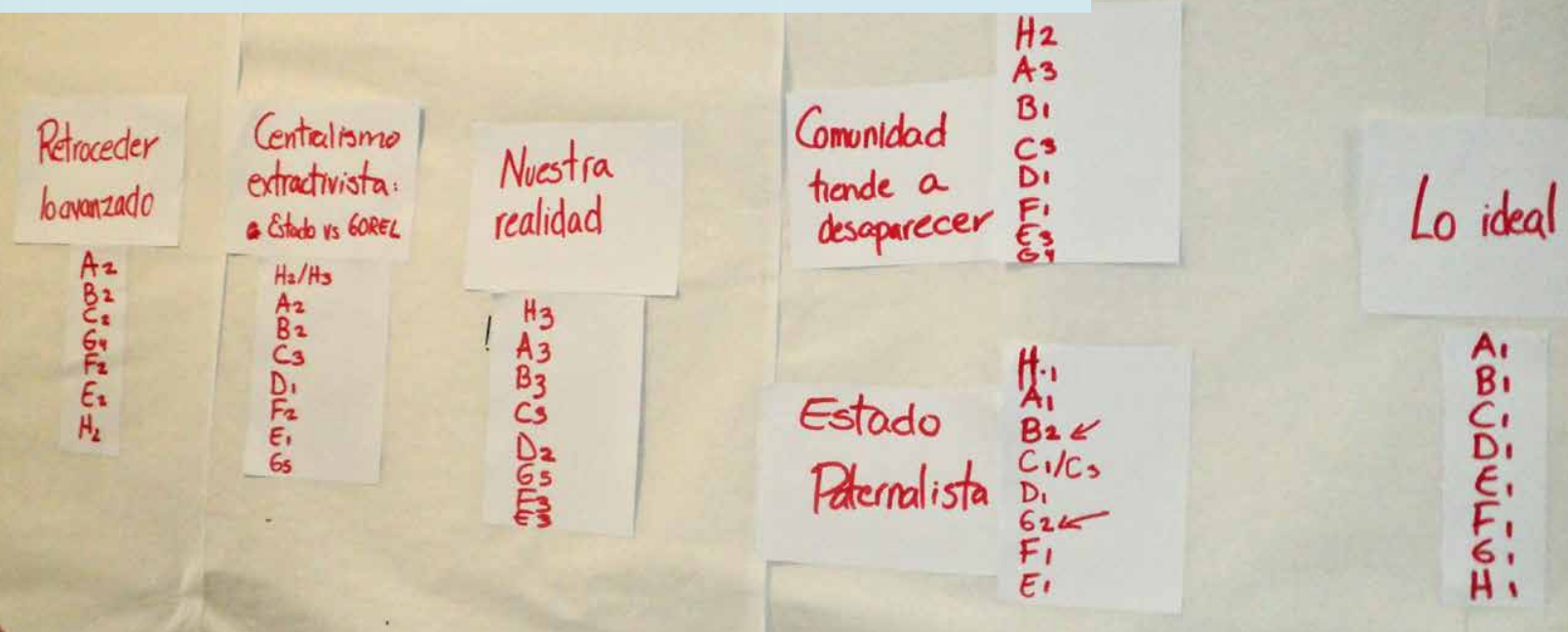


3. Répéter maintenant le processus pour les scénarios non souhaitables de la manière suivante :

a. Distribuer une fiche d'une couleur différente (p. ex., gris) aux participants en leur demandant d'inscrire un scénario non souhaitable sur chaque fiche.

b. Ramasser les fiches, rechercher les incompatibilités, éliminer les doublons et regrouper les scénarios pour délibérer.

c. Procéder de nouveau à une délibération pour sélectionner des scénarios contrastés. S'assurer qu'ils sont contrastés également par rapport aux précédents retenus. Placer les nouveaux scénarios sélectionnés avec les précédents.

4. Inviter les participants à imaginer des scénarios créatifs lors d'un troisième et dernier tour :

a. Distribuer une autre fiche de couleur (p. ex., jaune). Demander aux participants de trouver d'autres scénarios qui contrastent avec ceux déjà proposés. Cela stimule leur créativité.

b. Ramasser les fiches, éliminer les scénarios incompatibles ou en double, les regrouper et délibérer en les comparant aux scénarios déjà produits.

c. Placer les scénarios retenus avec les précédents.

\section{CONSEILS}

- Ce troisième tour n'est pas obligatoire. II n'est pas forcément possible d'envisager d'autres scénarios qui seraient différents et contrastés par rapport à ceux déjà identifiés. Ce tour peut aussi être facultatif si un nombre suffisant de scénarios contrastés ont déjà été évoqués lors des deux premiers tours.

- S'assurer que toutes les configurations se trouvent au moins une fois dans les scénarios.

- Les scénarios qui diffèrent en raison de plusieurs configurations de facteurs bien contrastées sont utiles pour étudier les avenirs plausibles. Par conséquent, il est extrêmement important de définir des configurations de facteurs bien contrastées, y compris des ruptures, pour aboutir à une diversité de scénarios contrastés et plausibles.

\section{Élaborer les notes de présentation* des scénarios}

Vérifier que les participants s'accordent sur le fait que les scénarios sont suffisamment contrastés et variés pour représenter un éventail d'avenirs plausibles. Décrire chaque scénario plus complètement (c.-à-d. rédiger une note de présentation) Les étapes de rédaction d'une note de présentation cohérente sont les suivantes : 
- Rédiger la trame du scénario en rassemblant dans un seul paragraphe la description complète des configurations de ses facteurs moteurs. C'est le point de départ qui représente une image précise de l'avenir.

- Pour chaque scénario, en gardant à l'esprit ce point de départ, compléter progressivement la note de présentation par les configurations des autres facteurs qui sont compatibles. Il est nécessaire d'identifier des configurations plausibles qui s'intégreront dans l'image d'ensemble que reflétera la combinaison des configurations des facteurs moteurs caractérisant chaque scénario.

- Ajouter d'abord les configurations des résultats (les facteurs situés dans le quadrant inférieur droit de la matrice) en commençant par les facteurs se trouvant en bas de ce quadrant sur la droite. Passer ensuite aux autres facteurs en tournant dans le sens inverse des aiguilles d'une montre.

- Quand les configurations compatibles des résultats sont marquées dans la note de présentation, l'image de l'avenir devient plus détaillée et précise. Ajouter les configurations compatibles des leviers (ceux qui se trouvent dans le quadrant supérieur droit et qui ne sont pas déjà utilisés dans la création du scénario). Commencer par les facteurs les plus bas à droite dans ce quadrant et continuer dans le sens inverse des aiguilles d'une montre. Ajouter les configurations compatibles des facteurs moteurs qui ne figureraient pas déjà parmi ceux indiqués.

- Quand ces configurations sont entrées, la note de présentation est presque prête. Compléter par les configurations compatibles des facteurs du peloton.

- À la fin du processus, pour chaque scénario, nous aurons une présentation ayant au moins un nombre de phrases égal au nombre de configurations de facteurs utilisé lors de son élaboration.

- Observer les autonomes et réfléchir pour voir comment ils pourraient s'intégrer au texte de présentation de chaque scénario.

\section{CONSEILS}

- Répartir le travail entre de petits groupes de participants. En général, des groupes de trois ou quatre personnes sont plus efficaces pour élaborer un scénario.

- S'assurer que chaque groupe partage le texte du scénario avec les autres.

- L'élaboration d'un scénario suit une séquence logique. Il commence par les facteurs, puis suivent les résultats, les leviers, les autonomes et le peloton. Mais le texte final n'est pas forcément dans cet ordre. Pour qu'un scénario soit cohérent et convaincant, le texte peut être organisé dans un ordre différent. 


\section{Illustration : Résultats de l'atelier de formation à la prospective qui a eu lieu en Indonésie, en Ouganda et au Pérou}

Au total, 25 scénarios ont été produits. Ces scénarios sont exposés en détail dans les rapports consultables ici : https:/www.cifor.org/gcs-tenure/publications/projectpublications/. Le tableau 10 présente les configurations futures plausibles de la sécurité du foncier forestier selon les avenirs décrits dans les scénarios. Certains scénarios sont regroupés, car les avenirs qu'ils évoquent sont similaires.

Ce tableau montre sept configurations différentes pour l'avenir de la sécurité foncière selon le type de relations entre les parties prenantes et la structure de pouvoir correspondante. Chaque configuration peut entraîner des situations différentes et contrastées :

- La configuration " harmonie » se trouve sur la première ligne du tableau. Là, la sécurité du foncier forestier est garantie pour tous grâce à des règles claires et respectées, avec des pouvoirs publics locaux et nationaux vigilants sur leur application.

- La configuration " communauté » (deuxième ligne) se caractérise par un régime foncier forestier géré par les communautés locales. Différentes situations surviennent selon la capacité des communautés à faire respecter leurs droits.

- Dans la configuration " échec de l'action de l'État" (troisième ligne), les groupes d'intérêts ont la possibilité de contrôler le régime foncier forestier. Un continuum de situations va d'une sécurité précaire du foncier forestier au conflit généralisé.

- La configuration " exclusion » (quatrième ligne) se caractérise par la prise de contrôle de la forêt par l'État. Aucune partie prenante n'est autorisée à utiliser les ressources forestières et la sécurité du foncier forestier.

n'est pas un problème. Cette configuration comprend aussi une situation où la population a quitté la zone (et par conséquent la sécurité du foncier forestier n'est plus un problème non plus).

- La configuration "égoïsme » se trouve aux lignes 5 et 6 du tableau. En l'absence de gestion communautaire ou sur des bases juridiques, ce sont les intérêts individuels ou privés qui prédominent. Cela conduit à des situations contrastées en fonction des acteurs qui détiennent suffisamment de pouvoir pour contrôler le foncier forestier et le défendre pour conserver l'accès et leurs " droits ».

- La configuration « marginalisation » qui se trouve à la ligne 7 du tableau correspond au contrôle d'une petite partie de la population (composée de l'élite et des personnes les plus aisées) sur la sécurité du foncier forestier. Les pauvres et les communautés locales sont dépossédés de leurs droits.

- La configuration "partenariat privé » à la ligne 8 correspond à une situation où la sécurité du foncier forestier est garantie grâce à l'action du secteur privé vis-à-vis de tous les usagers des forêts, sans intervention de l'État. 
Tableau 10. Avenirs plausibles de la sécurité du foncier forestier selon les scénarios élaborés au cours des ateliers de formation.

\begin{tabular}{|c|c|c|}
\hline Pérou & Indonésie & Ouganda \\
\hline $\begin{array}{l}\text { Minka (travailler ensemble)/ } \\
\text { Nous sommes heureux. Que }\end{array}$ & $\begin{array}{l}\text { Une réforme de rêve pour le } \\
\text { foncier forestier }\end{array}$ & $\begin{array}{l}\text { Le monde parfait } \\
\text { (renouvelable) }\end{array}$ \\
\hline $\begin{array}{l}\text { cela dure toujours! } \\
\text { La sécurité du foncier forestier } \\
\text { est garantie, les usagers } \\
\text { des forêts parviennent à un } \\
\text { consensus sur la propriété } \\
\text { des terres et exploitent les } \\
\text { ressources dans un contexte } \\
\text { d'harmonie et de réciprocité } \\
\text { ou sous la houlette de l'État. } \\
\text { Le régime foncier est clair et } \\
\text { compris de tous. Les usagers et } \\
\text { l'État bénéficient des produits } \\
\text { et des services procurés par les }\end{array}$ & $\begin{array}{l}\text { Les réformes du foncier } \\
\text { forestier peuvent se dérouler } \\
\text { sans conflit entre les parties } \\
\text { prenantes et confèrent de } \\
\text { la sécurité aux populations } \\
\text { dépendantes des forêts. }\end{array}$ & $\begin{array}{l}\text { Les droits de propriété } \\
\text { sur les terres et les } \\
\text { ressources forestières sont } \\
\text { incontestables, garantis } \\
\text { et protégés de façon } \\
\text { permanente par la loi et le } \\
\text { droit coutumier en vigueur } \\
\text { dans les communautés. Ils } \\
\text { sont reconnus et ne peuvent } \\
\text { être remis en question par } \\
\text { personne, ni aucun groupe ni } \\
\text { par l'État. }\end{array}$ \\
\hline
\end{tabular}
forêts.

\section{«Arc-en-ciel »}

Des droits fonciers clairs et respectés sont renforcés par les bonnes relations qui existent entre les usagers des forêts et par les autorités communautaires dont le rôle est central, car elles attribuent les droits et veillent à ce qu'ils puissent s'exercer normalement ; peu de conflits et les usagers bénéficient des produits et services procurés par les forêts.

\section{En route pour la catastrophe/} Le seul chemin

Le régime foncier des communautés est en péril en l'absence d'un soutien efficace sur le plan éducatif et politique qui permettrait de développer le potentiel local et de freiner l'action des groupes d'intérêt. Ces groupes ne manquent aucune occasion de faire main basse sur les ressources forestières, sinon leur avis n'est pas pris en compte dans les décisions publiques par un État autoritaire. On note une absence de communication entre les acteurs concernés.

\section{"Le pouvoir du peuple "}

La sécurité du foncier forestier est contrôlée par une communauté locale dominante, mais qui n'a pas une vue d'ensemble de la problématique.

\section{La mosaïque communautaire}

Les droits de propriété sur les terres et les ressources forestières sont garantis de manière aléatoire. Les communautés puissantes sont les seules aptes à garantir leurs propres droits sur le foncier forestier.

\section{Un long chemin pour la réforme du foncier forestier}

Il est difficile pour une communauté dépendant des forêts de parvenir à une certaine sécurité s'agissant du foncier forestier en raison d'un soutien insuffisant de la part du gouvernement et du secteur privé.

\section{Tous en chemin pour détruire Ia forêt avec l'aval du gouvernement}

Une politique discriminatoire contestée et contournée par tous favorise la remise en cause des droits de propriété sur les terres et les ressources forestières. Tout le monde peut défier n'importe qui, n'importe quel groupe et même l'État. Personne ne jouit d'aucune sécurité en matière de foncier forestier. 
Tableau 10. Suite

\begin{tabular}{|c|c|c|}
\hline Pérou & Indonésie & Ouganda \\
\hline La forêt désertée & \multirow[b]{2}{*}{$\begin{array}{l}\text { Le pouvoir du } \\
\text { gouvernement } \\
\text { Le gouvernement prend } \\
\text { le contrôle des terres } \\
\text { forestières. La sécurité du } \\
\text { foncier forestier perd de } \\
\text { l'importance ou n'est plus à } \\
\text { l'ordre du jour. }\end{array}$} & Mayantu \\
\hline $\begin{array}{l}\text { L'État est le seul propriétaire } \\
\text { foncier et contrôle toutes les } \\
\text { questions foncières. Personne } \\
\text { ne peut accéder aux ressources } \\
\text { forestières. La sécurité du } \\
\text { foncier forestier n'est plus une } \\
\text { question d'actualité. }\end{array}$ & & $\begin{array}{l}\text { Parce qu'il n'y a plus } \\
\text { de population, les } \\
\text { gouvernements locaux ont } \\
\text { l'exclusivité des droits fonciers } \\
\text { qui ne sont pas affectés par } \\
\text { les fluctuations du marché. }\end{array}$ \\
\hline "Moi d'abord " & \multirow{2}{*}{$\begin{array}{l}\text { L'emprise du secteur privé } \\
\text { sur la réforme du foncier } \\
\text { forestier }\end{array}$} & La loi de la jungle \\
\hline $\begin{array}{l}\text { Les droits fonciers sont } \\
\text { précaires, l'intérêt individuel }\end{array}$ & & \multirow[b]{2}{*}{$\begin{array}{l}\text { La réforme foncière est } \\
\text { demandée par tous ceux } \\
\text { qui sont préoccupés par la } \\
\text { dégradation des forêts et } \\
\text { la précarité de l'existence. } \\
\text { Les droits de propriété sur } \\
\text { les terres et les ressources } \\
\text { forestières ne sont pas } \\
\text { garantis; ils ne sont reconnus } \\
\text { ni protégés par aucune loi } \\
\text { ni aucun droit coutumier. } \\
\text { Seuls les corrompus et les } \\
\text { privilégiés ont des droits sur } \\
\text { le foncier forestier. }\end{array}$} \\
\hline $\begin{array}{l}\text { prévalant dans une ambiance } \\
\text { de conflit et de lutte pour } \\
\text { le contrôle de l'accès aux } \\
\text { ressources. Les droits fonciers } \\
\text { de certains groupes sans poids } \\
\text { économique sont bafoués, les } \\
\text { autorités se désintéressant de } \\
\text { leur fonction vis-à-vis des forêts. }\end{array}$ & $\begin{array}{l}\text { Précarité de la sécurité du } \\
\text { foncier forestier en raison } \\
\text { de conflits entre parties } \\
\text { prenantes. }\end{array}$ & \\
\hline
\end{tabular}

\section{Chaos et survie}

Dans le pays, les communautés ont accès au foncier forestier, mais la population attend plus de ressources financières, ce qui est source de désaccords et de conflits d'intérêts constants.

\section{Pas d'avenir pour la sécurité du foncier forestier}

Situation précaire du foncier forestier en raison d'un libre accès aux forêts et du fait que le ministère de l'Environnement et des Forêts a peu de pouvoir.

\section{Route vers la destruction de la forêt}

Les droits fonciers dans les forêts sont précaires, compte tenu de la prédominance de petits groupes qui veillent sur leurs intérêts ; les populations peu argentées en sont privées et seules les catégories aisées y ont accès.

\section{De l'échec de la réforme au capitalisme forestier}

Les droits fonciers dans les forêts sont précaires parce que la mise en œuvre de la réforme est aux mains du secteur privé.

\section{Chacun pour soi}

La sécurité du foncier forestier n'existe pour personne.

Les droits de propriété sur les terres et les ressources forestières ne sont pas garantis. Ils ne sont reconnus ni protégés par aucune loi ni aucun droit coutumier. Tout le monde a le droit de défier tout le monde.

\section{Les riches contre les pauvres}

Les droits de propriété sur les terres et les ressources forestières ne sont pas garantis pour tous en raison d'un système juridique discriminatoire et de la désuétude du droit coutumier. Le cadre juridique protège les riches et exclut les pauvres et les communautés locales (inégalité à l'égard de la sécurité du foncier forestier). 
Tableau 10. Suite

\begin{tabular}{lll}
\hline Pérou & Indonésie & Ouganda \\
\hline & Le secteur privé est aux \\
& manettes \\
& Les communautés peuvent \\
obtenir des droits sur les & terres forestières sans \\
réforme du foncier forestier.
\end{tabular}

\section{Encadré 16. Élaboration des scénarios}

\section{Le cas du Pérou}

Les participants ont défini des scénarios et rédigé la note de présentation à partir de la combinaison des configurations de facteurs. Ils ont éliminé les combinaisons de configurations qui étaient similaires et ont produit six scénarios différents pour chaque région.

\section{Le cas de l'Indonésie}

Les futurs scénarios ont été élaborés à partir des principaux facteurs moteurs. Pour chaque facteur moteur, on peut observer différentes configurations de variables. À Lampung comme à Maluku, les configurations de variables correspondaient à une situation souhaitable ou non, suivant la même tendance ou à une rupture. Il faut être attentif lors de ce processus, car il ne faut pas oublier de tenir compte de la configuration actuelle des variables. De nombreuses configurations de variables ne sont pas compatibles, ce qui signifie qu'elles ne peuvent survenir en même temps. À l'issue du processus, les participants ont commencé à élaborer les scénarios à partir des combinaisons des configurations de chaque variable. Les scénarios similaires ont été regroupés. À la fin de l'exercice, nous sommes arrivés à des scénarios contrastés. À Maluku, nous avions six scénarios et cinq à Lampung.

\section{Le cas de l'Ouganda}

À partir des principaux facteurs moteurs identifiés, leurs futures configurations (souhaitable ou non et rupture) ont été explorées. Les configurations incompatibles ont été définies par les participants et entrées dans la matrice « Incompatibilities ». Ensuite, les participants et les animateurs ont élaboré les divers scénarios à l'aide des différentes configurations de tous les principaux facteurs moteurs dont la compatibilité a été vérifiée dans la matrice. Chaque participant a eu l'occasion de produire des combinaisons possibles. La description des différentes configurations a ensuite servi à rédiger les notes de présentation des scénarios. Cette rédaction s'est déroulée en groupes de deux à quatre personnes. Le nombre de personnes dans le groupe dépendait du nombre de scénarios plausibles. Six scénarios ont été produits dans le district de Kibaale, sept dans celui de Lamwo et cinq dans celui de Masindi. 


\section{5 Étape 5 : Élaborer un plan d'action grâce à la prospective}

Lors d'un processus de coélaboration de scénarios, le point fondamental est de passer des scénarios à l'action. Les débats sur les scénarios doivent être diffusés et encouragés à l'extérieur du groupe d'experts qui a pris une part active à leur mise au point. Cela peut se faire en sollicitant les parties prenantes dans des débats publics ou en mettant sur pied des plans d'action grâce à la démarche rétrospective* ${ }^{*}$.

\subsubsection{Diffuser les scénarios et susciter le débat}

Nous utilisons des exemples de scénarios produits au niveau local dans trois pays dans le cadre du projet GCS-Tenure. Ces exemples peuvent illustrer un processus visant à mobiliser les parties prenantes locales pour imaginer les futures réformes foncières dans l'optique de parvenir à la sécurité foncière souhaitée par les communautés.

\section{Exemple de l'Indonésie ${ }^{1}$}

Le premier exemple est tiré d'une démarche prospective réalisée par des citoyens en Indonésie (provinces de Maluku et de Lampung) en 2015-2016. Il montre comment le fait de rendre publics les scénarios a conduit à un engagement pour créer des plans d'action.

La consultation publique a atteint trois objectifs. Elle a permis à des parties prenantes dans les districts et les provinces d'avoir connaissance des résultats de la PPA et de donner leur avis. Cela a assuré une adhésion et un soutien plus larges de ces plans en dehors des experts. Et l'aide du public a été sollicitée pour rédiger le plan d'action qui garantirait les droits fonciers au niveau local. Ce plan d'action pourrait être intégré aux programmes du gouvernement régional.

Le scénario futur le plus souhaitable à Maluku est "Une réforme de rêve pour le foncier forestier ". Ce scénario s'articule autour d'une gouvernance positive des terres et des ressources, dans la transparence, la responsabilisation, la coopération et la coordination. Il insiste aussi sur la reconnaissance des droits coutumiers. Il tient également compte de l'importance de l'allocation de fonds à la mise en œuvre de la réforme du foncier forestier. À Lampung, le scénario le plus souhaitable est "L'unité forestière d'aménagement face à la mondialisation ». Ce scénario présente l'unité forestière d'aménagement comme le principal lieu de la mise en œuvre de cette réforme au titre du programme de foresterie sociale. Les consultations ont rassemblé 40 personnes à Maluku et 36 à Lampung. Il s'agissait de représentants de différentes parties prenantes du district et de la province : agences responsables des forêts, du

1 Source : Nining Liswanti et Tuti Herawati, CIFOR Indonésie 
territoire, de la gestion des bassins versants, une entreprise publique, l'unité forestière d'aménagement, l'Agence de l'aménagement du territoire, les communautés, les ONG locales, les entreprises et universités locales.

La plupart des participants ont un point de vue différent sur la sécurité foncière au niveau local, notamment sur les droits fonciers dans les forêts. À Maluku, par exemple, il existe diverses lois et réglementations qui peuvent être du ressort de plusieurs agences et recouper le droit coutumier.

Les experts avaient une vision multidimensionnelle de la sécurité foncière. Ils ne la limitaient pas au faisceau de droits réellement accordés, mais considéraient qu'elle inclut les institutions et processus qu'ils estiment nécessaires pour l'exercice de droits garantis au niveau local. À Maluku, par exemple, la sécurité foncière comprend des modalités de gouvernance qui font partie des processus de mise en œuvre, ainsi que des interventions inscrites dans le scénario souhaité (bonne gouvernance, collaboration, respect et reconnaissance des institutions et du droit coutumier). Dans les scénarios non souhaitables, les situations données en exemple étaient sous le contrôle exclusif des acteurs dominants du gouvernement ou du secteur privé.

Que les situations soient souhaitables ou non, les scénarios mettaient en lumière les principales problématiques que rencontrerait la réforme du régime au bénéfice de la sécurité foncière des communautés locales de Maluku et de Lampung. Les contraintes pesant sur la mise en ouvre de cette réforme sont l'allocation d'un budget et des changements de politiques publiques et de réglementations. À Maluku, les autres contraintes sont la coordination, l'absence de données sur l'aménagement du territoire et la non-reconnaissance des droits coutumiers. À Lampung, les contraintes étaient les ressources humaines au niveau du gouvernement et de la communauté, le développement d'entreprises communautaires, comme le savoir, l'expérience et l'expertise de plusieurs acteurs en matière de résolution de problèmes. Ces facteurs seront très importants dans la mise en œuvre la réforme forestière et pourraient miner la sécurité foncière.

\section{Exemple du Pérou ${ }^{2}$}

Le Pérou a produit une série de dessins qui représentent les scénarios dégagés dans les deux régions. Ces dessins étaient basés sur les effets de chaque facteur évalué au cours de chaque scénario sur ces trois enjeux : l'état des forêts, la situation de la communauté et les relations entre la communauté et le gouvernement.

La figure 12 montre 6 des 11 dessins produits au cours des exercices PPA : Scénario " idéal " en haut à gauche, " mauvais " scénario en haut à droite, " quand les ONG se substituent au gouvernement » milieu gauche, «mi-chemin » milieu droit, " gouvernement paternaliste » en bas à gauche et " disparition de la communauté » en bas à droite.

2 Source : Alejandra Zamora et Iliana Monterroso, CIFOR Pérou 

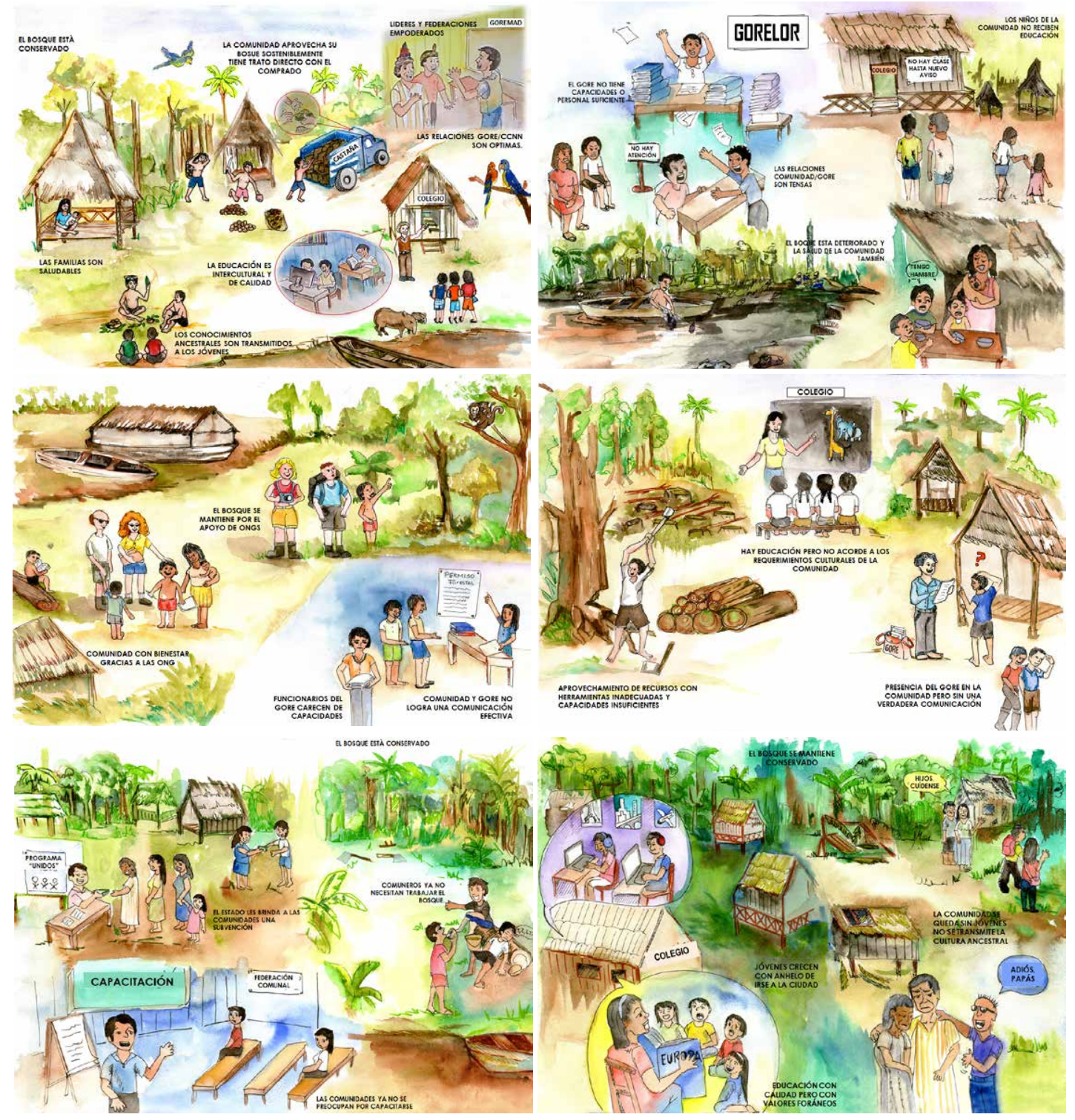

Figure 12. Six exemples de scénarios graphiques produits lors des ateliers PPA au Pérou.

Note : De haut en bas et de gauche à droite : « Notre idéal », « Retour au point de départ », « Quand les ONG prennent la place du gouvernement », « Mi-chemin », « un gouvernement paternaliste », «Disparition de la communauté ». 
Jusqu'ici, ces scénarios ont été présentés lors de divers événements à différents niveaux de gouvernance. Au congrès national forestier de 2016, la présentation a porté sur les différences entre les scénarios, qui sont influencés par l'industrie extractive, très importante dans chaque région. Seuls les scénarios de Loreto ont été présentés à la réunion de la Fédération des communautés autochtones du Napo moyen, de Curaray et Arabela (FECONAMNCUA) ; cette réunion a rassemblé des chefs des communautés autochtones du bassin du Napo. Le scénario de "l'État paternaliste » a retenu leur attention. Les participants s'accordaient sur le fait que ce serait exactement ce qui se passerait s'ils recevaient une pension mensuelle comme l'indique ce scénario. Enfin, au cours de l'atelier qui a eu lieu avec l'organisation nationale des femmes autochtones andines et amazoniennes du Pérou (ONAMIAP), le débat sur les scénarios a porté sur le rôle des femmes dans le régime de propriété foncière collective. Le scénario sur la disparition de la communauté a particulièrement attiré l'attention, car les participants sont nombreux à redouter ce cas de figure. Ces scénarios venaient à l'appui de la proposition de renforcer l'identité autochtone de chaque communauté et de lutter contre le mépris de cette culture.

Les échanges sur ces scénarios ont mis en évidence les caractéristiques différentes de la sécurité foncière dans les régions. Parmi les différents facteurs de l'insécurité foncière, les parties prenantes locales et régionales considéraient que ce sont les facteurs politiques et sociaux qui affectent les droits fonciers des communautés autochtones. L'absence d'informations et de coordination a été évoquée comme la contrainte la plus importante dans la reconnaissance des droits collectifs. L'équipe qui veille à la mise en œuvre a prévu d'autres événements pour continuer à diffuser les résultats afin de mobiliser les parties prenantes à différents niveaux de gouvernance, notamment au niveau national.

\section{Exemple de l'Ouganda ${ }^{3}$}

En Ouganda, avant d'organiser l'atelier PPA pour les femmes, la démarche a été exposée lors de six ateliers avec 19 hommes et 5 femmes. Le manque d'implication des femmes dans les exercices était dû au fait qu'elles connaissent peu les questions de régime foncier, notamment des forêts ; la plupart des femmes en Ouganda ne prennent pas part à ces questions parce que, traditionnellement, elles ne possèdent pas de terre. Il leur était aussi difficile de s'absenter de la maison plusieurs jours par semaine pendant deux semaines, et de s'engager à suivre l'ensemble des sessions PPA.

Chacun des trois ateliers a été suivi par 15 femmes, représentant le gouvernement local, des organisations de la société civile et diverses catégories de la communauté locale. Ces ateliers PPA destinés à donner des moyens d'agir aux femmes et à prendre en compte leurs préoccupations concernant la question du foncier forestier ont été organisés dans les trois districts de Kibaale, Masindi et Lamwo. Ces ateliers visaient à identifier un éventail de scénarios sur la sécurité du foncier forestier qui affectaient les femmes et à élaborer un ensemble d'actions correctives. Les objectifs spécifiques de l'atelier PPA étaient les suivants :

3 Source : Concepta Mukasa, CIFOR Ouganda 
- Identifier les facteurs clés qui affectent la sécurité foncière des femmes et leur articulation avec la mise en œuvre de la réforme.

- Identifier les actions possibles pour atténuer les répercussions négatives et promouvoir des transformations positives.

- Débattre collectivement pour réfléchir, d'une part, à la mise en ouvre de la réforme et au moyen de la rendre plus performante en matière de sécurité foncière pour les femmes et, d'autre part, aux actions possibles y afférentes.

Les résultats issus des trois districts montrent que les femmes préferent que la réforme $\mathrm{du}$ foncier forestier se déroule dans un délai plus court, p. ex., cinq ans au lieu de 10 ou plus. En ce qui concerne la menace qui pèse sur la sécurité du foncier forestier, la plupart des femmes ne sont pas autorisées à posséder de la terre en Ouganda. Même si elles plantent des arbres, ceux-ci ne leur appartiennent pas. Par conséquent, dans la plupart des cultures ougandaises, les droits fonciers des femmes sont restreints. Pour leur ouvrir plus de droits en la matière, il faut que les hommes les soutiennent pour qu'elles puissent posséder des ressources financières et de la terre, comme prendre des décisions. Les femmes évoquent aussi les rapports familiaux (mari/épouse, épouse/ belle-famille, enfants et parents) qui ont aussi une incidence positive ou négative sur leur situation à l'égard de la sécurité du foncier forestier.

Les principaux facteurs sont variés et ont des définitions diverses. Les femmes sont surtout concernées par des causes qui les touchent de près. Une fois le consensus atteint, huit facteurs principaux ont servi à élaborer les scénarios, puis la base d'un plan d'action : influence politique, dynamique démographique, mise en œuvre et respect des politiques et programmes publics, capacité technique, accès aux ressources financières, marchés et industrialisation, niveau de sécurité foncière et influence des ONG. Le facteur "Influence des ONG » concerne uniquement les femmes parce que la plupart des ONG s'attachent à promouvoir l'égalité entre les sexes et en général les actions en faveur des femmes. Leur soutien peut appuyer la cause de la sécurité foncière des femmes.

La réflexion sur le plan d'action pour impliquer les femmes dans la mise en ouvre a surtout porté sur plusieurs facteurs. Par exemple, en ce qui concerne "l'influence politique ", les femmes attendaient une action susceptible de les encourager à se présenter à des élections au suffrage direct. Cela leur permettrait d'être plus nombreuses dans les conseils et au parlement, plus présentes dans les prises de décisions et d'accroître les indemnités pour les femmes qui œuvrent en faveur de la discrimination positive. Concernant le facteur « Mise en ouvre et respect des politiques et programmes publics ", les femmes, à titre individuel et en groupe, ont besoin de soutien pour planter des arbres (p. ex., fourniture de semences et de plants). Le soutien des femmes devant s'impliquer dans les décisions était un problème dans le cas du facteur "Accès aux ressources financières ». Quant au facteur "Influence des ONG ", il est important de créer un environnement favorable pour inciter plus d'organisations à aider les femmes à sécuriser leurs droits fonciers sur les forêts. 
Le principal problème est d'élaborer à partir de la situation actuelle le cheminement vers les scénarios futurs, chacun composé d'étapes concrètes et cohérentes. Cela permettra de définir les actions nécessaires pour augmenter les chances de parvenir à un scénario souhaité, comme celles qui empêcheraient l'occurrence de scénarios non souhaitables.

Grâce à la rétrospective, en partant de la fin pour remonter au début, il est possible de déterminer et d'organiser logiquement toutes les actions nécessaires pour s'assurer que la situation finale se réalisera. Cette méthode permet d'éviter le risque de définir des actions à court terme qui semblent conduire à un avenir souhaité, mais ayant des effets inattendus et indésirables par la suite.

Dans une démarche rétrospective, le groupe d'experts suit les étapes préliminaires suivantes :

- Définir une série de scénarios sur l'avenir de la sécurité du foncier forestier avec des facteurs bien identifiés et leurs diverses configurations possibles (résultats de la coélaboration des scénarios).

- Avec le groupe d'experts, caractériser la situation actuelle de la sécurité du foncier forestier au niveau local par rapport aux facteurs identifiés lors de l'élaboration des scénarios.

- Préparer un grand espace de travail pour afficher les situations actuelles et futures, en les séparant bien (voir la photo).

- Sélectionner un des scénarios de sécurité du foncier forestier. Sur la gauche de l'espace de travail, afficher la configuration du premier facteur. Sur la droite de l'espace de travail, afficher la configuration actuelle de ce premier facteur.

\section{Procédure}

Lorsque les étapes préliminaires sont achevées, engager une réflexion avec les participants en suivant la figure 13. Voici une idée pour amorcer cette réflexion :

« Nous sommes dans l'avenir (p. ex., en 2030) et voici actuellement la situation de la sécurité du foncier forestier (en montrant le côté gauche de l'espace de travail) par rapport à ce facteur. Il y a quinze ans (en 2015), la situation de la sécurité du foncier forestier était celle-ci par rapport à ce même facteur. Je suis historien et je voudrais faire le compte rendu de ce qui s'est passé entre 2015 et 2030 afin que l'on comprenne les raisons de cette transformation. Écrivez sur les fiches tous les événements qui sont survenus pour expliquer ce changement. »

Suivre ensuite les étapes ci-dessous :

- Ramasser toutes les fiches des participants. Les lire à haute voix et les placer dans l'espace de travail à un endroit plus ou moins proche de la future situation ou de la situation actuelle, selon leur contenu. La position exacte des premières fiches importe peu, car on pourra les déplacer. 
- Chaque fois qu'on ajoute une fiche, discuter avec les participants pour décider de la placer soit avant, soit après, soit au même endroit que les autres fiches. Si l'événement inscrit sur la fiche doit avoir lieu avant les autres événements déjà définis, placer cette fiche à droite de l'autre fiche/des autres fiches. Si l'événement inscrit sur la fiche doit avoir lieu après, placer cette fiche à gauche. Si l'événement est simultané, placer la fiche exactement au-dessus ou au-dessous de l'autre/ des autres. Si l'événement est similaire, éliminer la fiche.

- Quand toutes les fiches sont placées dans l'espace de travail, l'opération est terminée.

- Avec les participants, réfléchir aux liens qui existent entre les fiches en partant de l'avenir (à gauche) pour aller vers le présent (à droite). Le débat porte sur la question suivante : "le lien entre les éléments est-il direct ou faut-il que quelque chose survienne entre ces éléments, et si oui, qu'est-ce ? Inscrire les événements nécessaires le cas échéant.

- Pour chaque lien, identifier les principaux acteurs et leur rôle.

- Enregistrer les résultats en les notant par écrit et en utilisant des techniques visuelles (photos de l'espace de travail, diapositives...).

- Répéter la démarche rétrospective pour le facteur suivant du même scénario, jusqu'au dernier.

- Répéter cette démarche pour chaque scénario comme l'indique la figure suivante (étapes 1 à 4).

Les résultats de cette démarche rétrospective servent ensuite à amener les participants à déterminer les éléments stratégiques et à concevoir un plan d'action grâce à la prospective.

Les éléments les plus stratégiques correspondent aux actions qui, simultanément, éviteront les scénarios non souhaitables et favoriseront les scénarios souhaitables (étape 5 de la figure 13). Un plan d'action peut ensuite être préparé. Définir l'ordre de mise en œuvre des éléments stratégiques, en partant de la situation actuelle pour aboutir à l'avenir espéré. Il est nécessaire pour cela que les parties prenantes s'accordent sur la nature de l'avenir espéré/souhaitable pour la sécurité du foncier forestier. À cette fin, utiliser à la fois la démarche rétrospective, la diffusion des scénarios et la promotion de débats publics.

\section{Encadré 17. Comparaison Rétrospective/Planification}

L'avantage de la rétrospective repose sur le type de questions posées aux suivante : «Que faudrait-il faire pour atteindre la situation souhaitée ? » Les personnes répondent en général de façon linéaire avec un cheminement fondé sur une logique déterministe et En rétrospective, la question est la suivante : «Que doit-il se passer pour que cette situation espérée devienne ce type de question, les personnes identifient d'abord les points d'étape. Le parcours entre ces points d'étape déterminera le cheminement. 


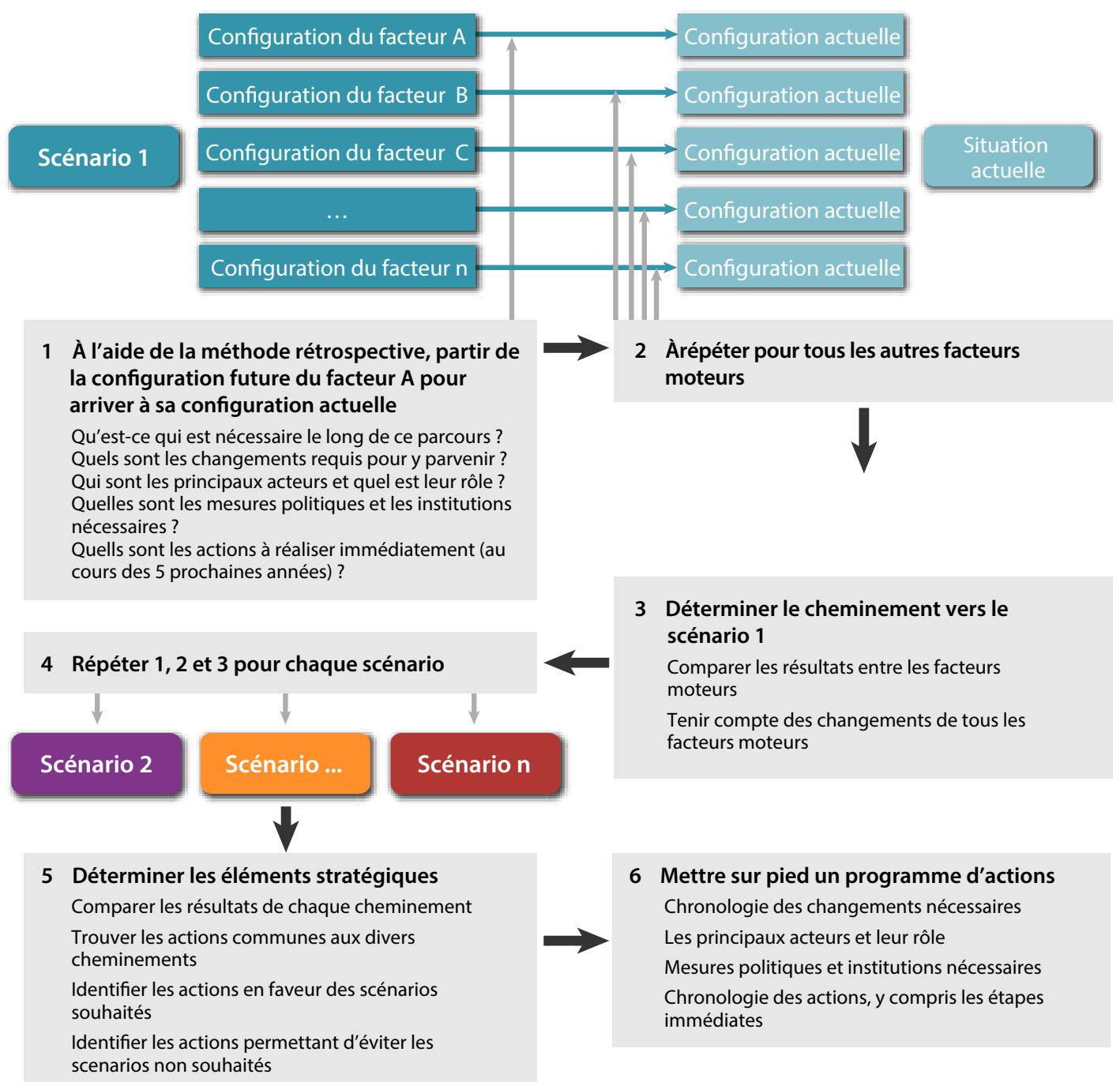

Figure 13. Les étapes de la démarche rétrospective.

En raison de la nature des ateliers de formation qui ont eu lieu en Indonésie, en Ouganda et au Pérou, les participants n'avaient pas le temps de produire des supports de communication ou de rédiger un plan d'action (ils devaient aussi élaborer des plans pour la mise en œuvre sur le terrain d'une démarche prospective sur la sécurité du foncier forestier). Par conséquent, au Pérou, les participants n'ont réalisé qu'un travail de rétrospective. Leur expérience a permis d'affiner la démarche rétrospective comme indiqué plus haut. 


\section{Bibliographie}

Bourgeois R and Jesus F. 2004. Participatory prospective analysis: exploring and anticipating challenges with stakeholders. CGPRT Publication (ESCAP) No. 46. Bogor: ESCAP.

Bourgeois R, Penunia E, Bisht $S$ and Boruk D. 2017. Foresight for all: Co-elaborative scenario building and empowerment. Technol. Forecast. Soc. Chang. 124, 177-188. doi:10.1016/j.techfore.2017.04.018

Liswanti N. 2012. Building a shared vision: Scenarios for collaborative land use planning in Central Moluccas Regency, Indonesia. The Futures of Agriculture. Brief No. 39-English. Rome: Global Forum on Agricultural Research.

Mwangi E and Meizen-Dick R. 2009 Understanding property rights in land and natural resource management. In Kirsten JF, Dorward AR, Poulton C and Vink $\mathrm{N}$, eds. Institutional Economics Perspectives on African Agricultural Development. Washington, DC: International Food Policy Research Institute. 295-318.

Poffenberger M. (ed.) 1990. Keepers of the Forest: Land Management Alternatives in Southeast Asia. Westford, CT: Kumarian Press.

Shantiko B. 2012. Seeking Harmony: Scenarios for nature conservation and agricultural development in Kapuas Hulu district, Indonesia. The future of agriculture. Brief No. 18-English. Rome: Global Forum on Agricultural Research.

Shantiko B, Liswanti N and Bourgeois R. 2017. Scenarios building in complex commons: Challenges, opportunities and lessons learned in engaging multiple stakeholders to improve land use decisions in Indonesia. Bogor, Indonésie : Center for International Forestry Research.

Wollenberg E, Edmunds D and Buck L. 2000. Anticipating Change: Scenarios as a Tool for Adaptive Forest Management, A Guide. Bogor, Indonésie : Center for International Forestry Research. 


\section{Annexes}

\section{Annexe 1. Suggestions des participants pour la mise en œuvre de la PPA sur la sécurité du foncier forestier}

\section{Mise en œuvre pratique sur le terrain}

- Mettre en œuvre immédiatement tandis que l'atelier PPA est encore frais dans les esprits, organiser les activités PPA près du lieu d'hébergement, prévoir le déplacement des personnes qui en sont chargées, veiller à ce que les responsables du projet assurent le suivi auprès des parties prenantes et apporter les conseils pratiques nécessaires aux personnes chargées de la mise en œuvre, y compris assurer le suivi de la mise en œuvre sur le terrain.

- Prévoir le calendrier de la mise en ouvre de cette démarche pour optimiser le résultat, scinder le processus en ateliers séparés. Les stagiaires planifient la mise en œuvre au niveau local grâce à deux ateliers séparés au moins.

Commentaires sur les suggestions : Sur le premier point, le formateur appuie la suggestion des participants. Le second point est laissé à l'appréciation de la direction du projet GCS. Le formateur soutient totalement et encourage la mise en œuvre sur le terrain grâce à deux ateliers au moins selon les plans proposés par les participants.

\section{Choix de l'animateur, des participants et du groupe d'encadrement}

De nombreuses suggestions ont été faites concernant la dimension humaine pour assurer la réussite de la mise en œuvre de la PPA :

- Choisir des " experts » et des participants impliqués selon leur expertise du sujet (connaissances), et aussi leur volonté de collaborer (attitude/ comportement).

- Faire participer les communautés locales aux ateliers au lieu de se baser sur l'opinion qu'on en a ou de les faire représenter par d'autres personnes en vue.

- Choisir l'animateur avec soin pour assurer la neutralité de l'atelier et l'explication claire de l'objectif grâce à sa maîtrise du sujet (car on ne peut expliquer que ce que l'on connaît parfaitement).

- Veiller à la coordination avec les représentants des pouvoirs publics et des organisations communautaires.

Commentaires sur les suggestions : Les suggestions confirment que les stagiaires ont bien compris l'importance du groupe d'experts qui va coélaborer les scénarios et les modalités du choix de ses membres. Ils ont aussi compris les qualités qui leur sont demandées en tant qu'animateurs. Ils font la distinction entre les experts et les autorités dont le soutien est nécessaire pour la mise en œuvre. 


\section{Annexe 2. Glossaire des termes de prospective utilisés dans ce document}

Anticipation

Fait de percevoir d'avance la réalisation d'une situation, la prévoir et, à l'occasion, fait de s'y préparer.

Avenir

Temps à venir

Calendrier

Totalité de la période considérée dans une étude du futur, en allant du passé vers l'avenir.

Causalité

Lien logique entre les événements dans lequel une cause précède un effet et le fait de modifier la cause change l'effet.

Cheminement

Trajectoire dans le temps, correspondant à une suite particulière d'actions et de conséquences sur fond d'évolutions autonomes, ce qui induit une situation future précise.

Connaissances préalables Informations sur l'avenir avant qu'il n'existe, obtenues par l'étude du futur.

Expert

Personne disposant de compétences, de connaissances, de discernement ou d'un savoir-faire dans un domaine particulier grâce à la recherche scientifique, à son expérience, à sa perspicacité ou à son métier.

Exploration

Grâce à l'anticipation, étude d'un vaste éventail de possibilités d'évolutions futures, envisagées sous divers angles de vue.

Facteur

Agent ou élément à l'origine d'un changement, qui affecte ou détermine l'avenir.

Horizon temporel

Incertitude

Échéance la plus lointaine dans l'avenir, qui est considérée dans une étude du futur.

État résultant de l'impossibilité de connaître totalement l'avenir.

Note de présentation Description cohérente d'un scénario (ou d'une famille de scénarios), qui indique ses principales caractéristiques et sa dynamique, les relations entre les principaux facteurs de changement et leurs conséquences. 
Planification stratégique

Plausible

Prévision

Prospective

Proactif

Rétrospective

Ruptures

Scénario

Statu quo

Système

Tendance

Transition
Action qui consiste à préparer une situation future.

Jugé raisonnable à cause des hypothèses de départ, de la cohérence interne et du lien logique avec la réalité.

Affirmation de la survenue d'une situation à l'avenir, souvent fondée sur les connaissances et les tendances actuelles.

Démarche systématique, participative et multidisciplinaire visant à explorer l'avenir à moyen et à long terme ainsi que les facteurs de changement. La prospective en France est basée sur le principe que l'avenir n'est pas écrit, mais à construire collectivement .

Orienté vers l'action bien avant une future situation, afin de provoquer la réalisation d'un avenir souhaitable et d'éviter un avenir non souhaitable.

Démarche qui consiste à réfléchir à partir de la définition d'un avenir possible pour déterminer ce qui doit arriver afin qu'il devienne réalité et relier cela au présent.

Changements brusques et importants par rapport à la nature d'une tendance ou à son orientation.

Description d'une possible situation future en fonction d'un ensemble explicite, cohérent et logique d'hypothèses sur les principales relations et les facteurs de changement.

Cheminement vers l'avenir qui s'inscrit dans la continuité de la situation actuelle.

Ensemble d'éléments interdépendants qui sont organisés en structure cohérente.

Orientation générale d'un mouvement ou d'un changement au fil du temps.

Transformation du système qui passe d'une configuration à une autre. 
Qualitatif

Quantitatif

Vision
Caractérise quelque chose qui peut être observé, mais pas mesuré quantitativement.

Caractérise quelque chose qui peut être observé et mesuré, et dont la quantité peut être déterminée.

Image éloquente de l'avenir (en général souhaité).

Références plus détaillées consultables ici : http://www.fao.org/docs/eims/ upload/315951/ Glossary\%20of\%20Terms.pdf 

Le Centre de recherche forestière internationale (CIFOR) a lancé en Indonésie, en Ouganda et au Pérou le projet GCS-Tenure, une étude comparative mondiale sur la réforme du foncier forestier. Ce projet a vocation à comparer le régime foncier coutumier et celui qui est inscrit dans la loi, et à étudier l'incidence de leur rapport sur la sécurité foncière des communautés qui dépendent des forêts, notamment des femmes et autres groupes marginalisés. En procédant à des comparaisons à l'échelle mondiale et à l'aide de méthodologies standardisées, cette étude analyse les différences quant à la réussite ou à l'échec des innovations destinées à renforcer la sécurité des droits fonciers, qu'elles soient institutionnelles ou concernent les politiques publiques. Elle permet aussi d'examiner comment ces innovations déterminent les stratégies susceptibles de conduire aux résultats désirés.

L'analyse prospective participative (PPA) est une première étape qui permet de mobiliser les principales parties prenantes. Grâce à des réunions participatives, tous les experts définissent et élaborent progressivement un éventail de scénarios de sécurité foncière. Ils conçoivent ensuite les actions correspondant aux scénarios définis. En appliquant la PPA, l'équipe de chercheurs vise à répondre aux questions suivantes : Quels sont les principaux facteurs qui influencent la sécurité du foncier forestier ? Quelles sont les actions possibles pour atténuer les conséquences néfastes (ou aplanir les obstacles qui freinent la mise en œuvre) et favoriser un changement positif (p. ex., un accès équitable pour les femmes et les groupes marginalisés) ? Qui doit être responsable de ces actions ? L'analyse prospective participative est destinée à aider les instances de décision à comprendre les facteurs clés, les difficultés et les conséquences futures des choix stratégiques.

cifor.org/fr

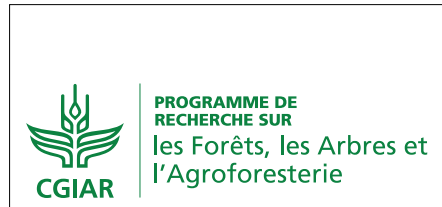

Cette recherche a été menée par le CIFOR dans le cadre du Programme de Recherche du CGIAR sur les Forêts, les Arbres et l'Agroforesterie (FTA). Le FTA constitue le plus important programme global de recherche pour le développement visant à amplifier la contribution des forêts, des arbres et de l'agroforesterie au développement durable, à la sécurité alimentaire et à la lutte contre le changement climatique. Le CIFOR dirige le FTA en partenariat avec Bioversity International, le CATIE, le CIRAD, I'ICRAF, I'INBAR et TBI.

Les travaux du programme FTA sont soutenus par le Fonds fiduciaire du CGIAR : cgiar.org/funders
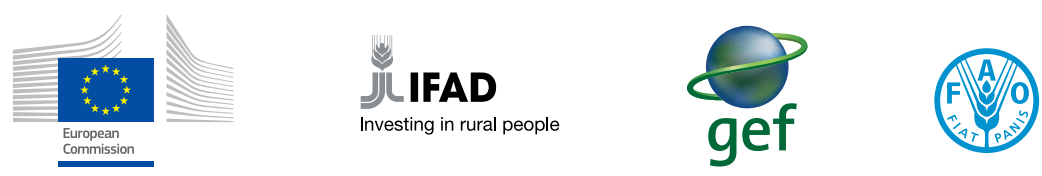

Organisation des Nations Unies pour I'alimentation et l'agriculture

\section{Centre de recherche forestière internationale (CIFOR)}

Le CIFOR contribue au bien-être humain, à l'équité et à l'intégrité de l'environnement en réalisant des travaux de recherche novateurs, en renforçant les capacités de ses partenaires et en nouant le dialogue avec tous les acteurs afin d'éclairer les politiques publiques et les pratiques qui touchent les forêts et les populations. Le CIFOR est un centre de recherche du CGIAR et dirige le Programme de recherche du CGIAR sur les forêts, les arbres et l'agroforesterie (FTA). Le siège du CIFOR est à Bogor, Indonésie, avec des bureaux à Nairobi, Kenya ; Yaoundé, Cameroun ; Lima, 